\title{
Metal-Free, Mild, and Selective Synthesis of Bis(pyrazolyl)alkanes by Nucleophile-Catalyzed Condensation
}

\author{
Maxym Tansky, Zipeng Gu, Robert J. Comito* \\ Department of Chemistry, The University of Houston, 4800 Calhoun Road, Houston, \\ Texas 77004 \\ Corresponding author: rjcomito@central.uh.edu
}

Supporting Information

\section{Contents}

$\begin{array}{ll}\text { 1. Spectral Data for Pyrazoles } & \text { S2 }\end{array}$

2. Spectral Data for Bis(azolyl)methanones $\quad$ S4

$\begin{array}{ll}\text { 3. Spectral Data for Bis(azolyl)alkanes } & \text { S18 }\end{array}$

4. Initial Optimization Studies in Toluene S68

5. Hammett Analysis and Mechanistic Data $\quad$ S69 
1. Spectral data for Pyrazoles

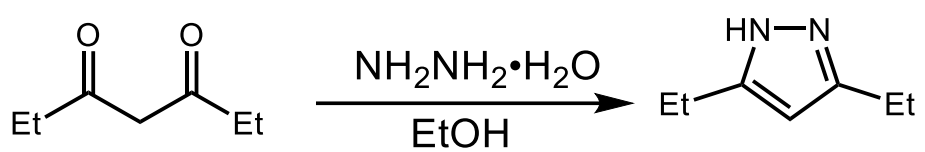

\section{3,5-diethyl-1H-pyrazole}

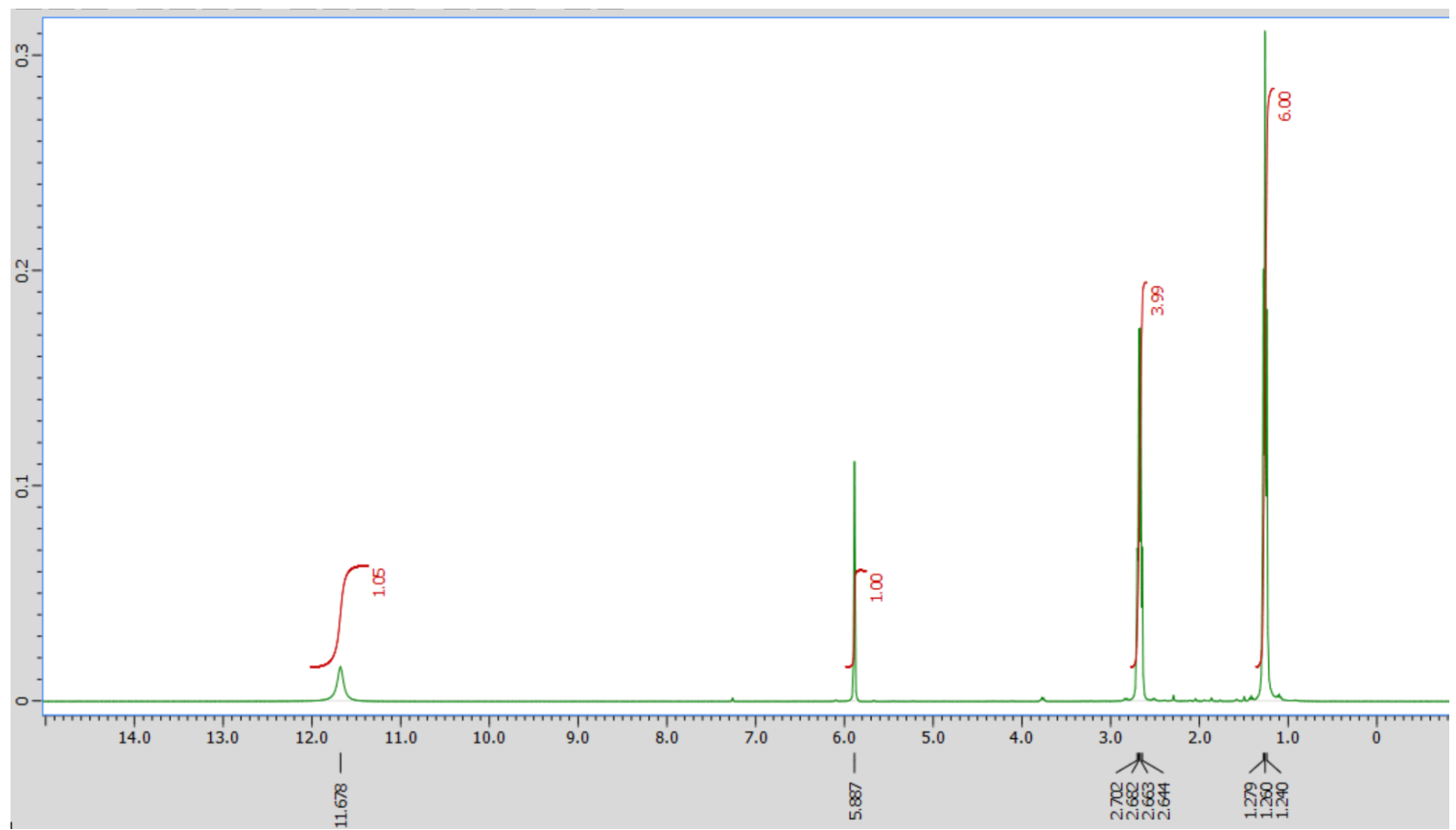

Figure $\mathrm{S} 1.1{ }^{1} \mathrm{H}$ NMR (400 MHz, $\mathrm{CDCl}_{3}$ ) spectrum of 3,5-diethyl-1H-pyrazole

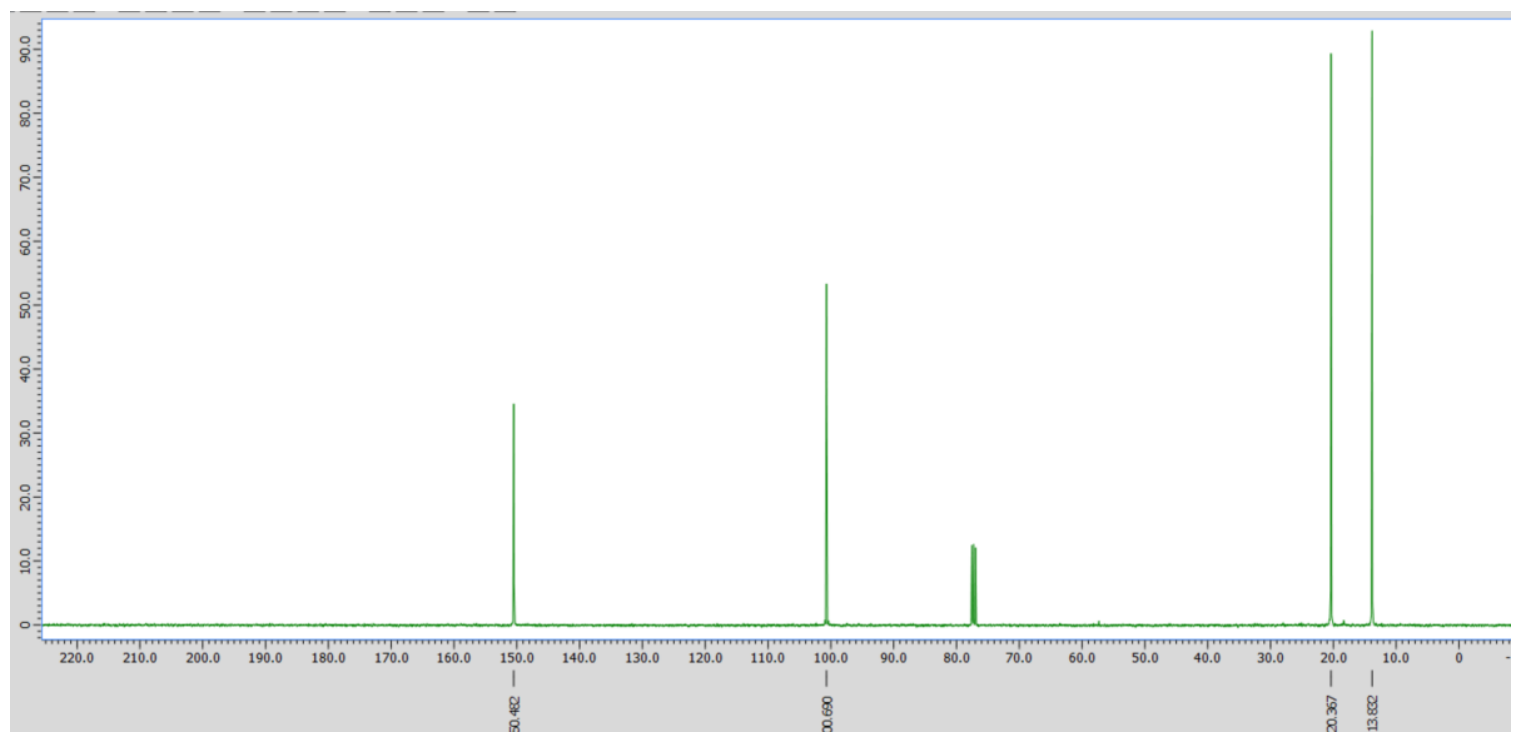


Figure S1.2 ${ }^{13} \mathrm{C}$-NMR $\left(101 \mathrm{MHz}, \mathrm{CDCl}_{3}\right)$ spectrum of 3,5-diethyl-1H-pyrazole<smiles>CC(=O)c1cc(C)cc(C)c1C(C)=O</smiles>

\section{3-mesityl-1H-pyrazole}

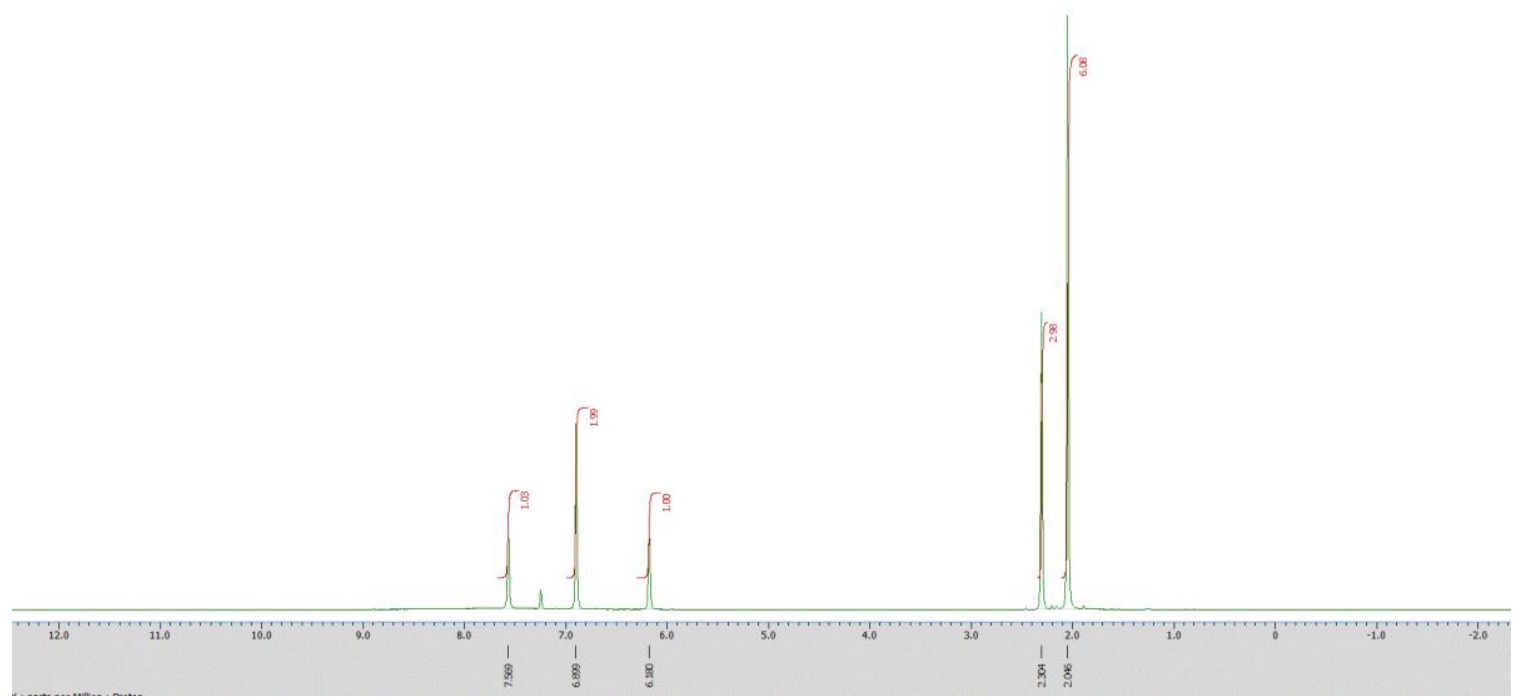

Figure S1.3 ${ }^{1} \mathrm{H}$ NMR (400 MHz, $\mathrm{CDCl}_{3}$ ) spectrum of 3-mesityl-1H-pyrazole

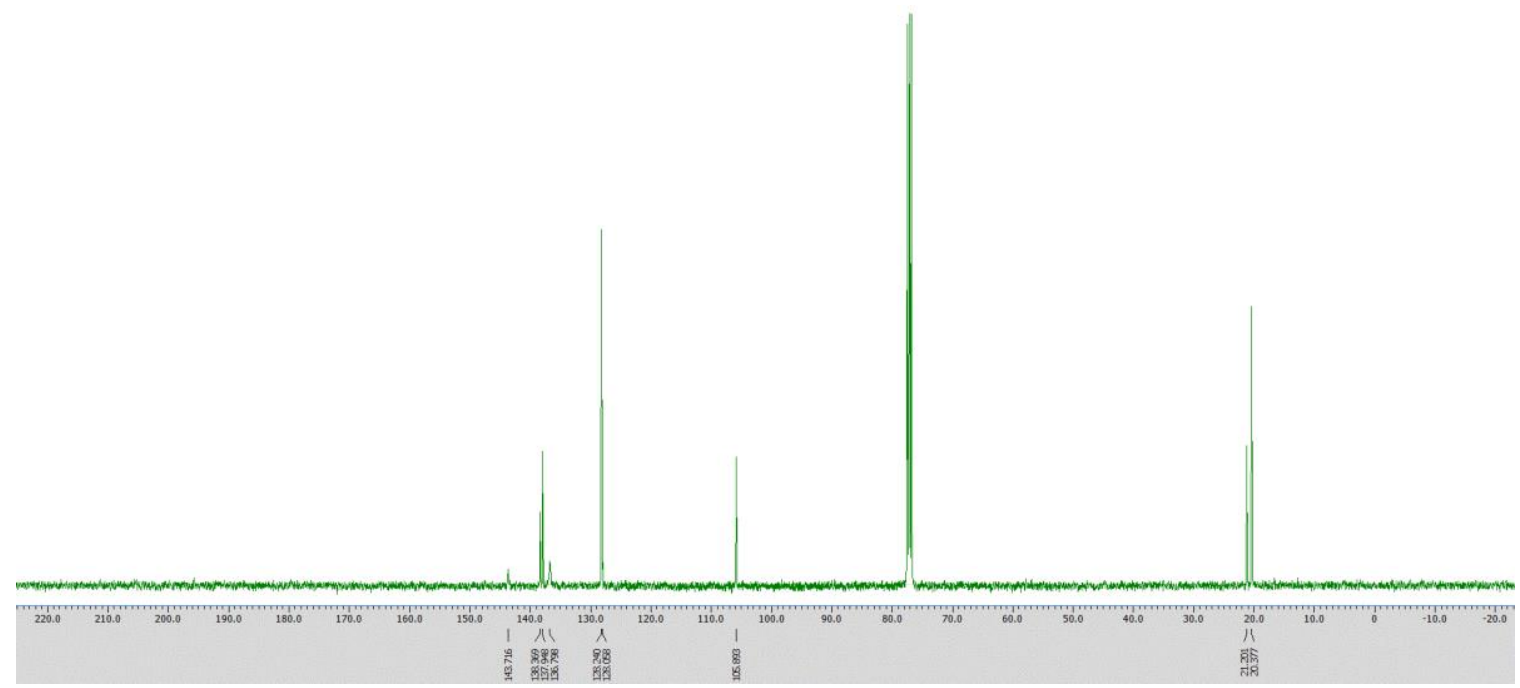


Figure $\mathrm{S} 1.4{ }^{13} \mathrm{C}$ NMR $\left(100 \mathrm{MHz}, \mathrm{CDCl}_{3}\right)$ spectrum of 3-mesityl-1H-pyrazole

\section{Spectral data for Bis(azolyl)methanones}

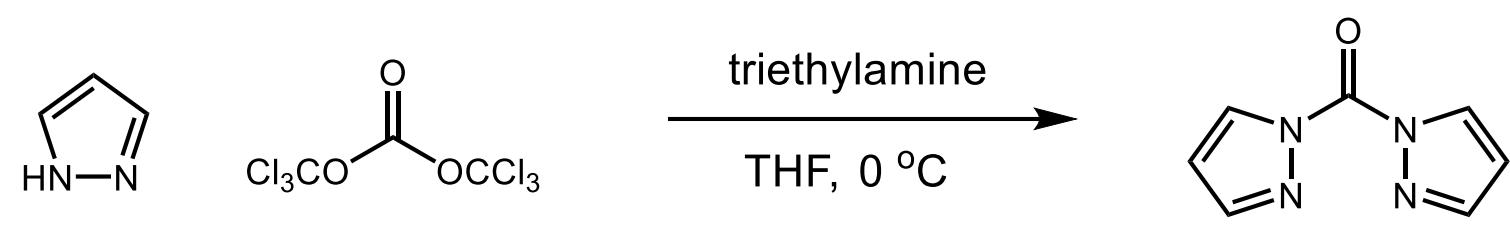

bis(pyrazol-1-yl)methanone

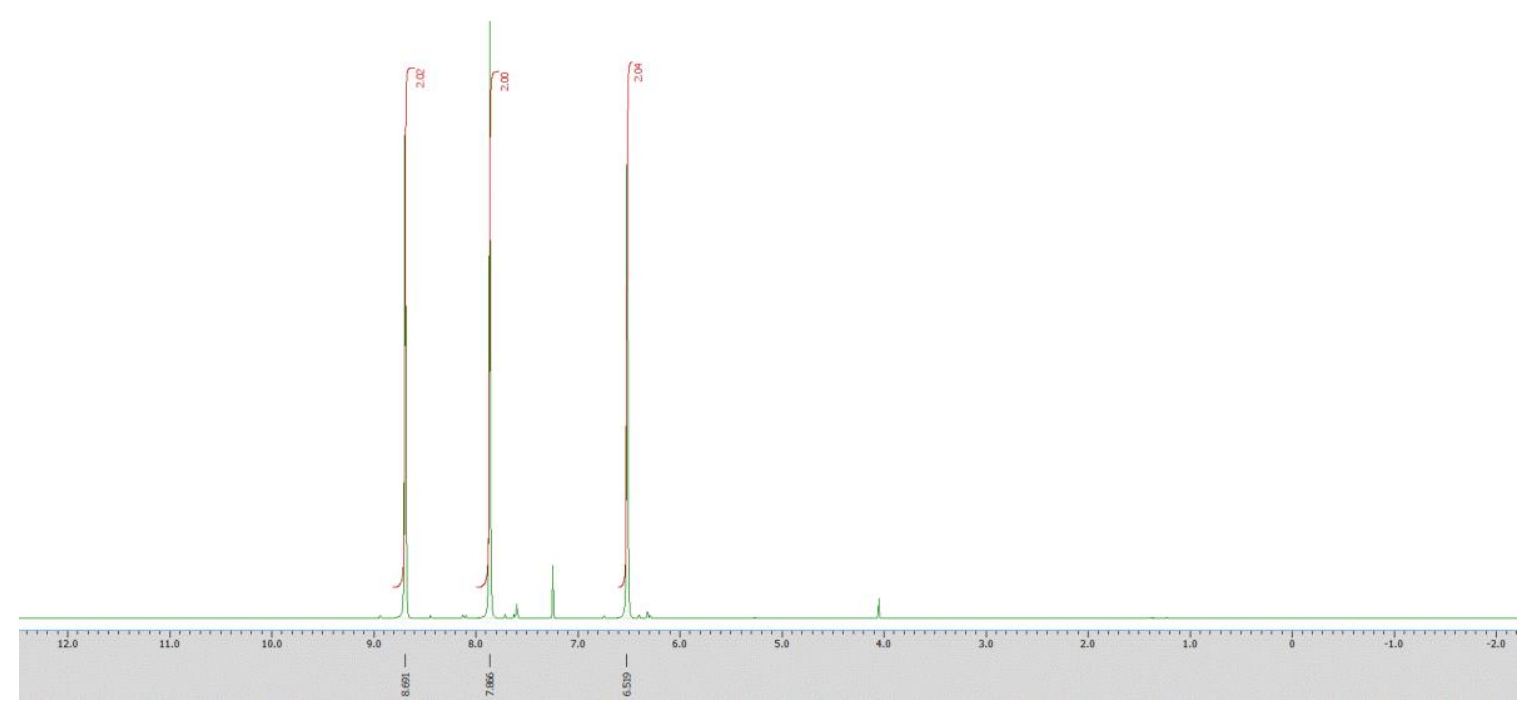

Figure $\mathrm{S} 2.1{ }^{1} \mathrm{H}$ NMR (400 MHz, $\mathrm{CDCl}_{3}$ ) spectrum of bis(pyrazol-1-yl)methanone 


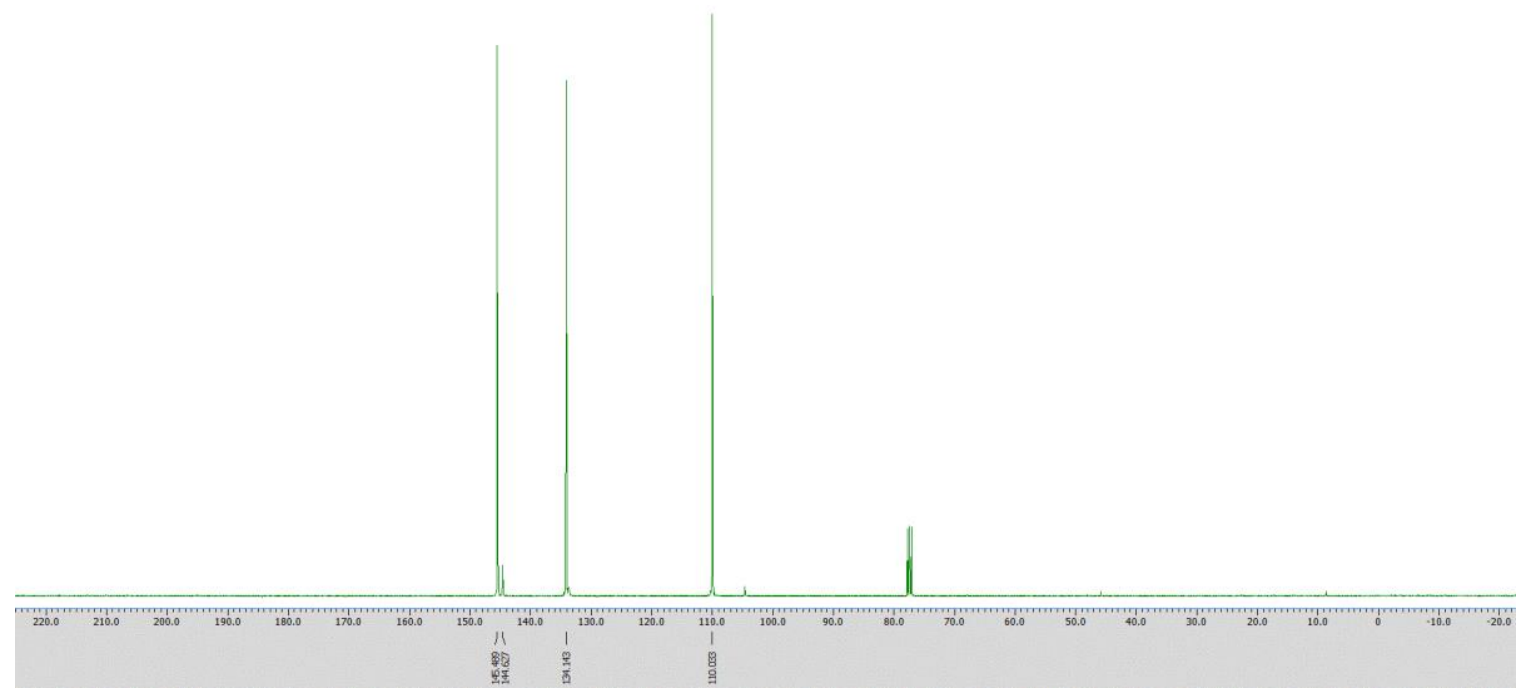

Figure S2.2 ${ }^{13} \mathrm{C}$ NMR $\left(101 \mathrm{MHz}, \mathrm{CDCl}_{3}\right.$ ) spectrum of bis(pyrazol-1-yl)methanone

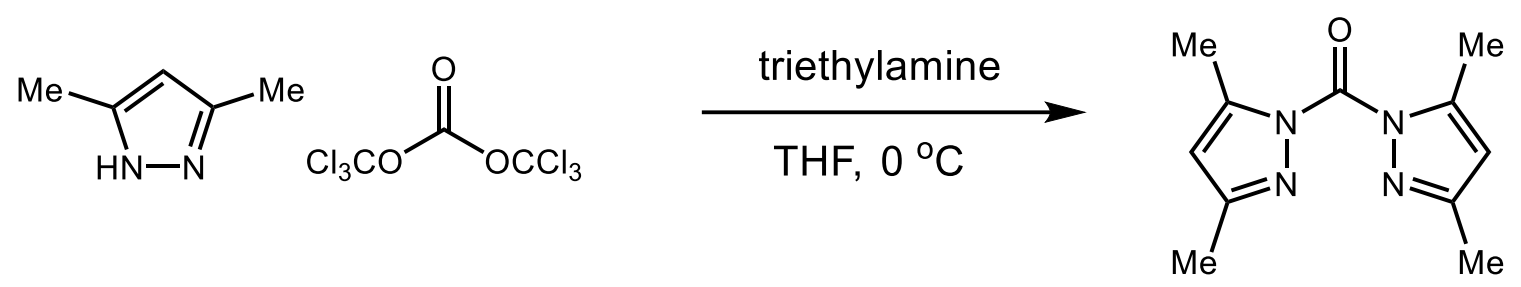

\section{bis(3,5-dimethylpyrazol-1-yl)methanone}

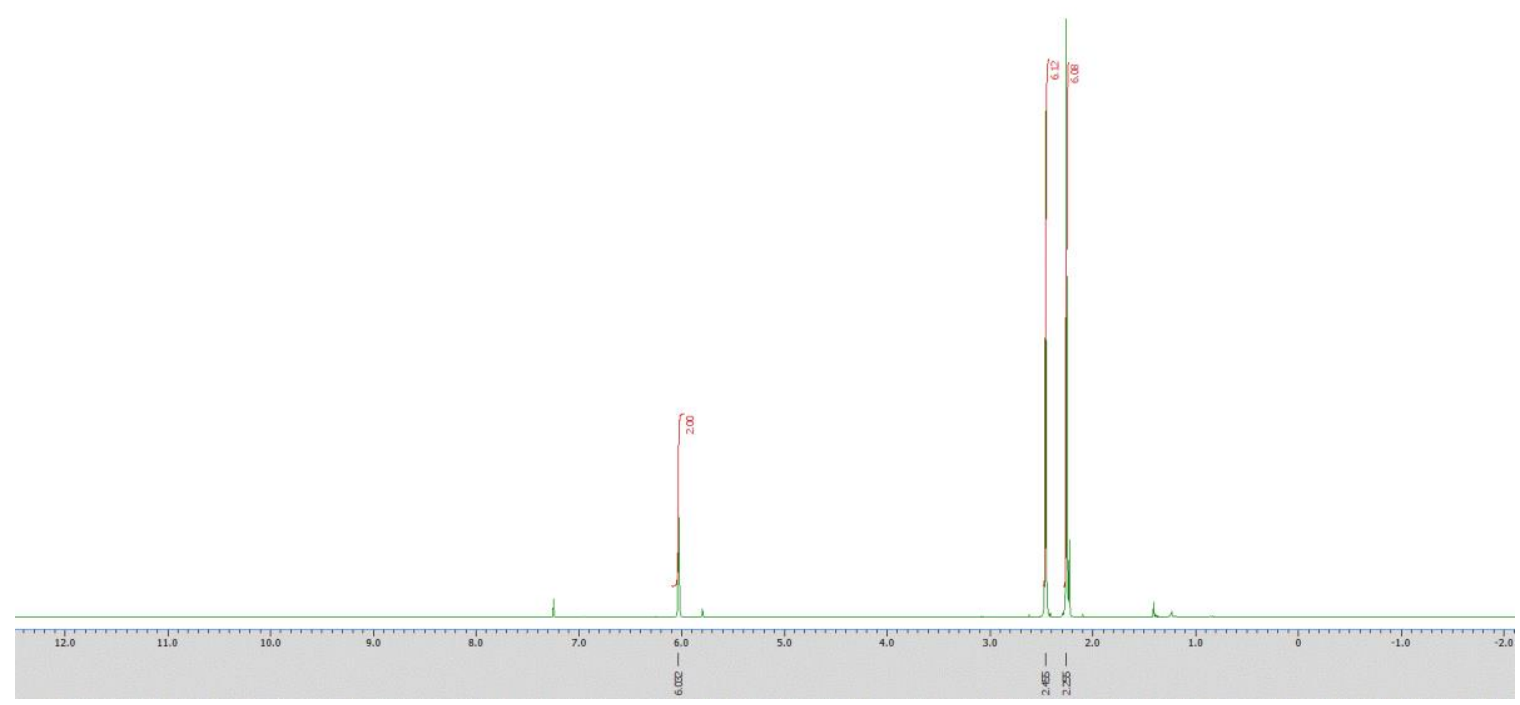


Figure $\mathrm{S} 2.3{ }^{1} \mathrm{H}$ NMR $\left(400 \mathrm{MHz}, \mathrm{CDCl}_{3}\right)$ spectrum of bis(3,5-dimethylpyrazol-1yl)methanone

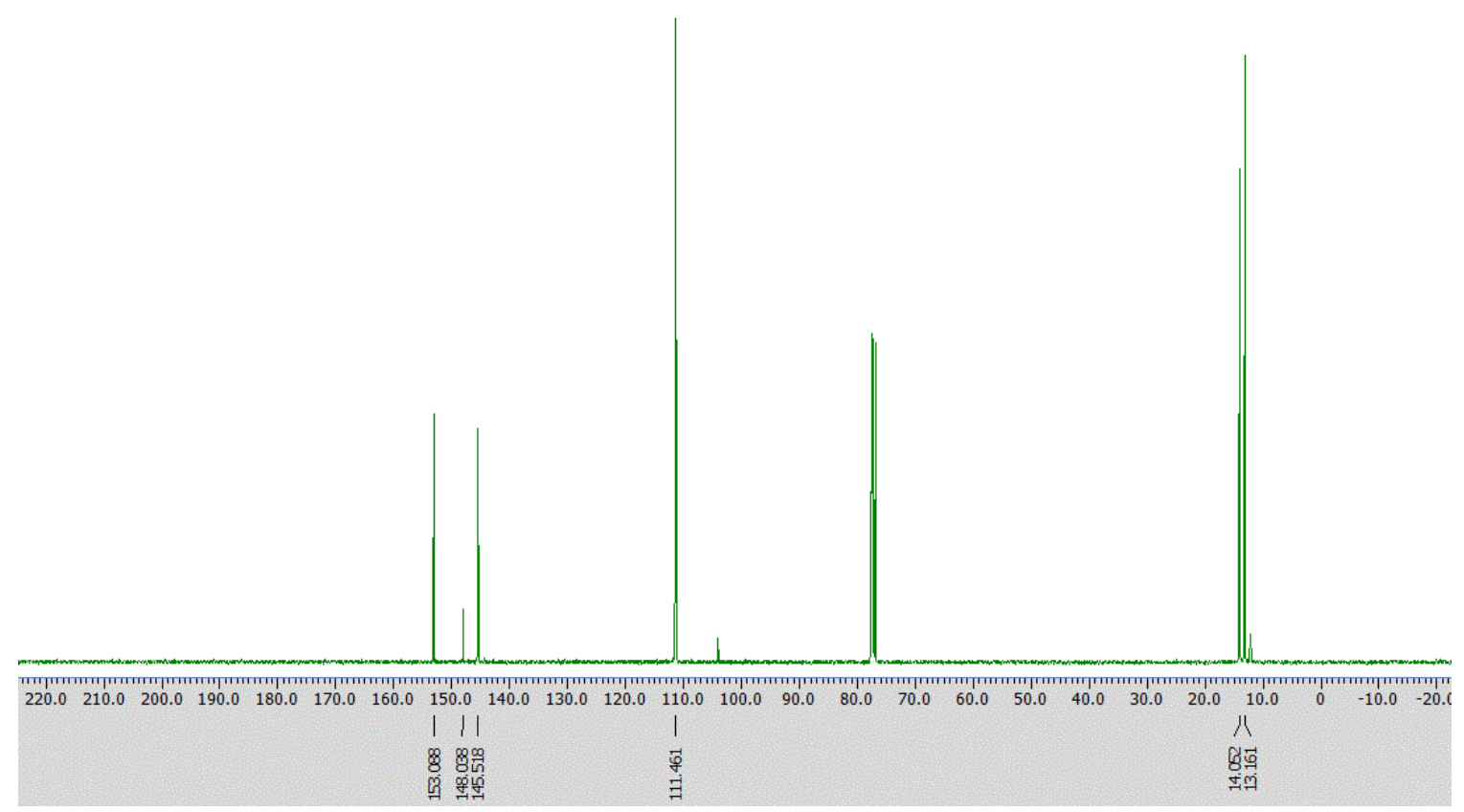

Figure $\mathrm{S} 2.4{ }^{13} \mathrm{C}$ NMR $\left(100 \mathrm{MHz}, \mathrm{CDCl}_{3}\right)$ spectrum of bis(3,5-dimethylpyrazol-1yl)methanone<smiles>CCc1cc(CC)[nH]n1</smiles><smiles>O=C(OC(Cl)(Cl)Cl)OC(Cl)(Cl)Cl</smiles>

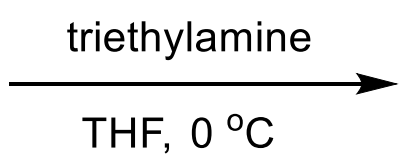<smiles>CCc1cc(CC)n(C(=O)n2nc(CC)cc2CC)n1</smiles>

\section{bis(3,5-diethylpyrazol-1-yl)methanone}




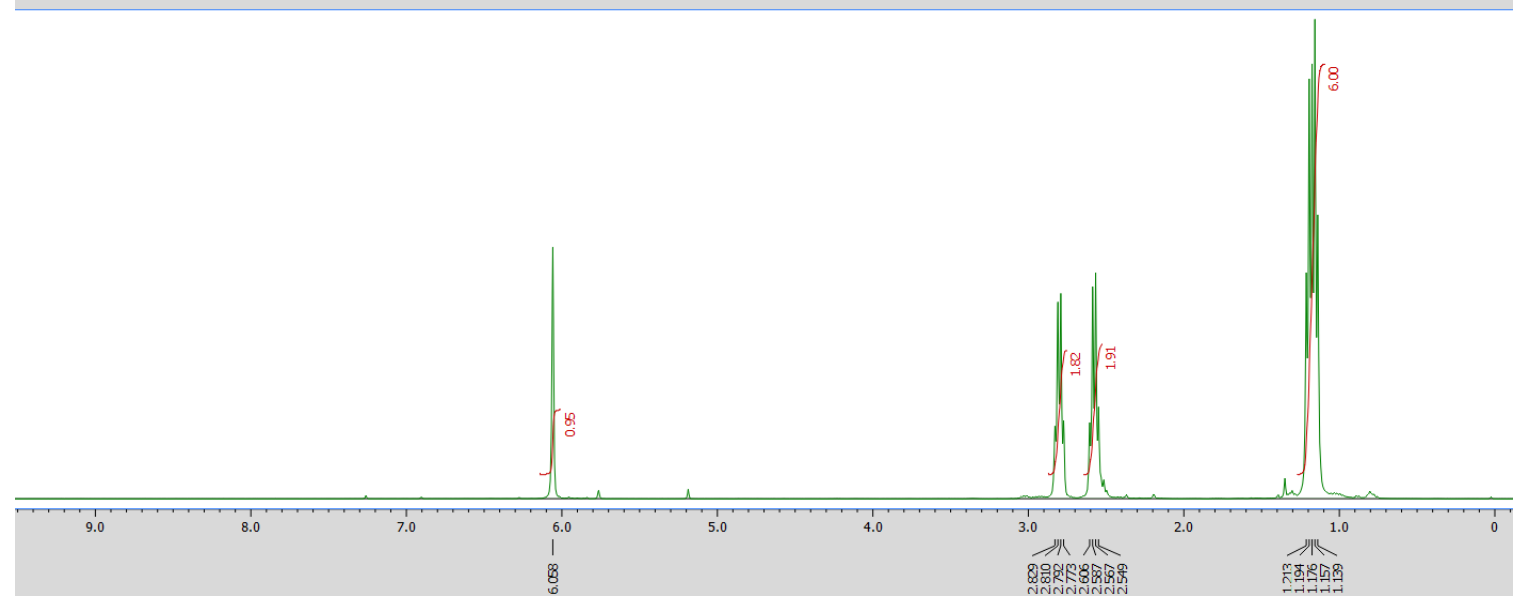

Figure $\mathrm{S} 2.5{ }^{1} \mathrm{H}$ NMR (400 $\left.\mathrm{MHz}, \mathrm{CDCl}_{3}\right)$ spectrum of bis(3,5-diethylpyrazol-1yl)methanone

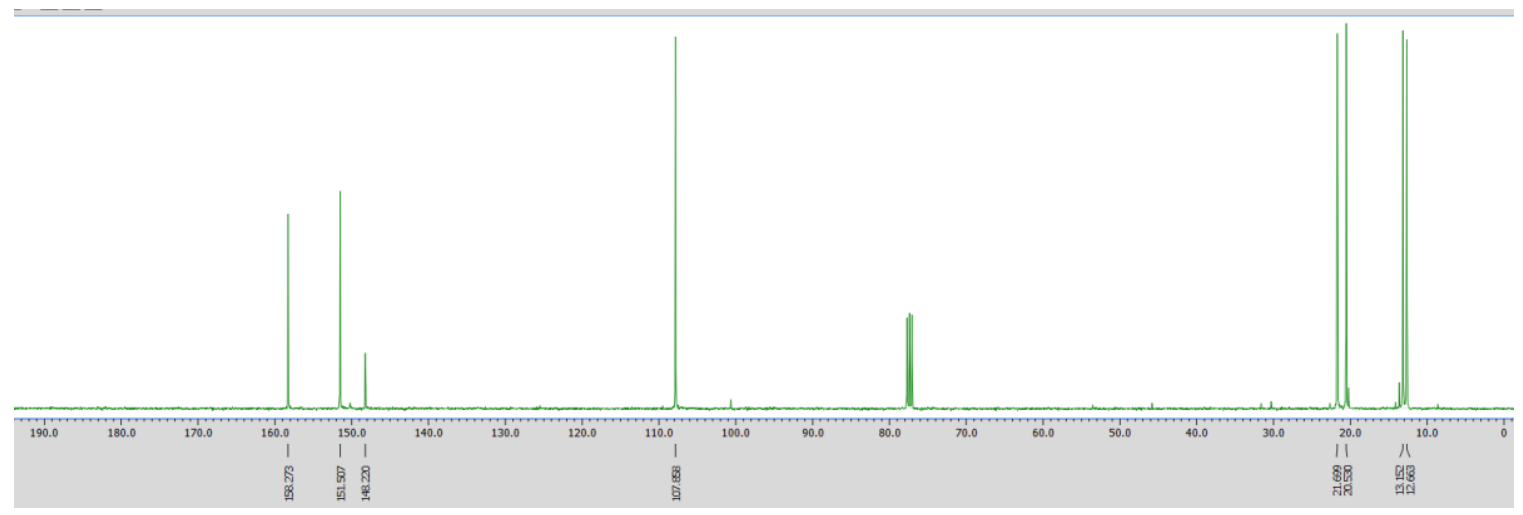

Figure $\mathrm{S} 2.6{ }^{13} \mathrm{C}-\mathrm{NMR}\left(101 \mathrm{MHz}, \mathrm{CDCl}_{3}\right)$ spectrum of bis(3,5-diethylpyrazol-1yl)methanone 


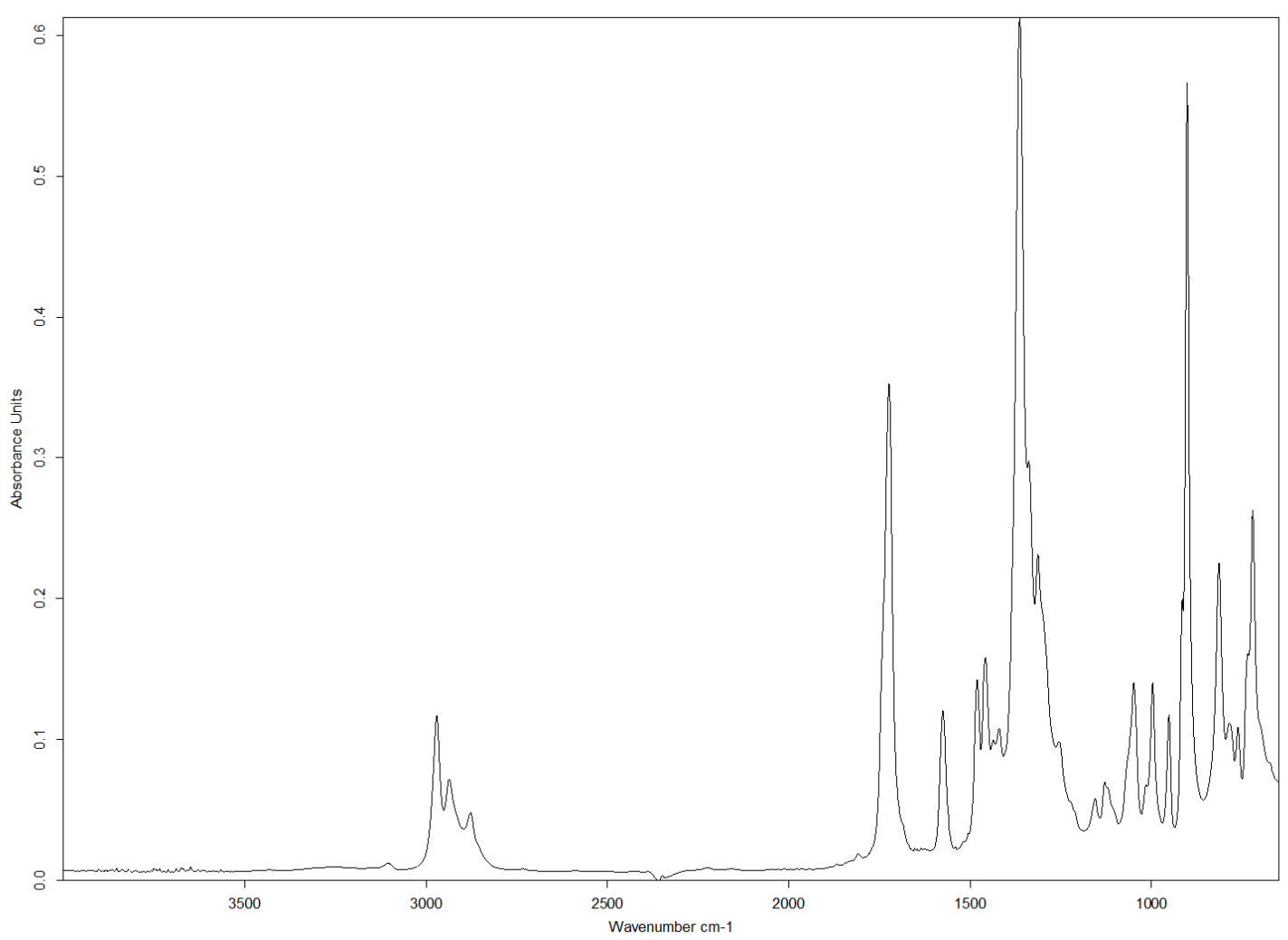

Figure S2.7 IR (Diamond ATR) spectrum of bis(3,5-diethylpyrazol-1-yl)methanone

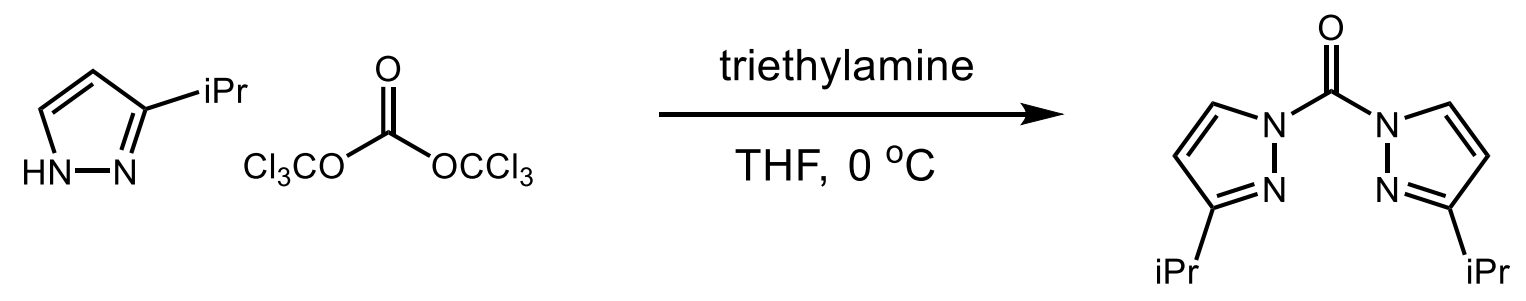

bis(3-isopropylpyrazol-1-yl)methanone 


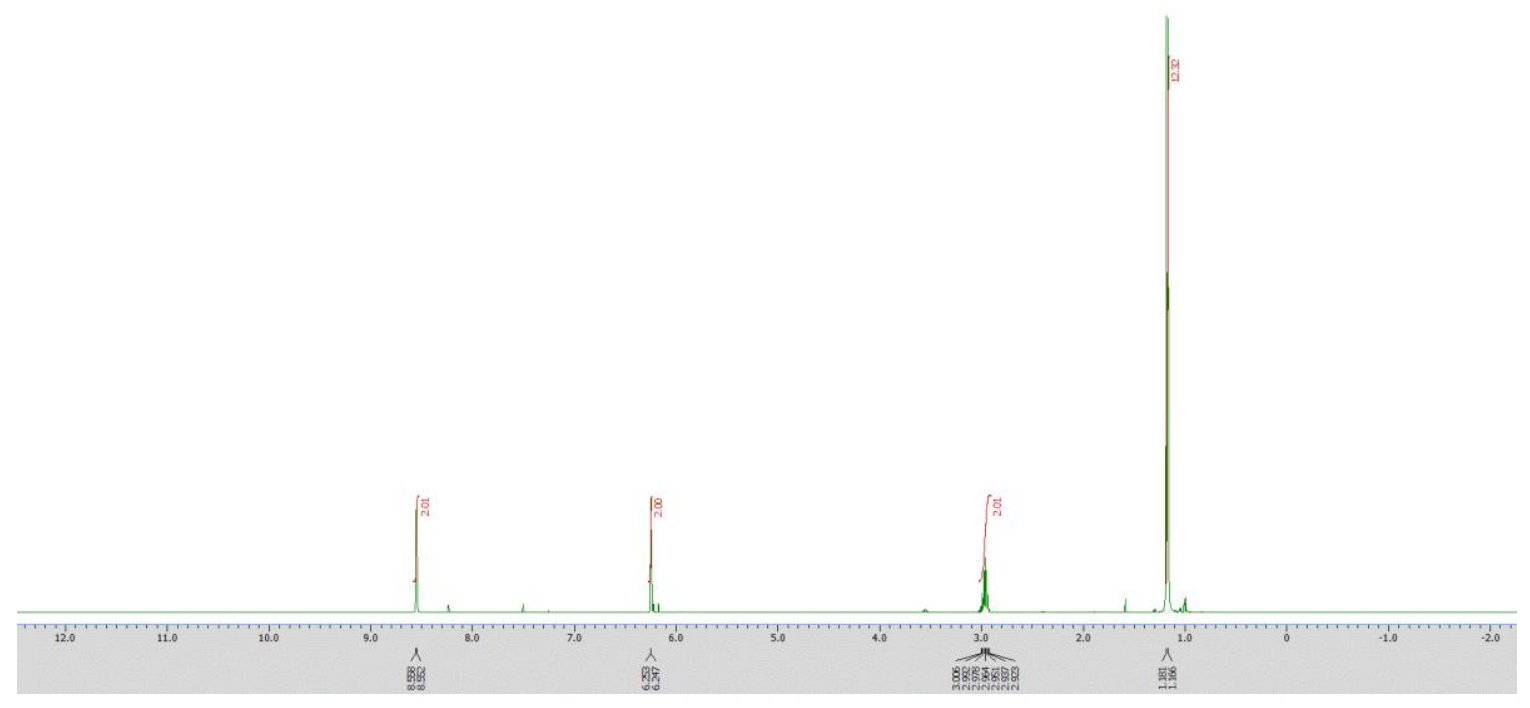

Figure $\mathrm{S} 2.8{ }^{1} \mathrm{H}$ NMR (400 $\left.\mathrm{MHz}, \mathrm{CDCl}_{3}\right)$ spectrum of bis(3-isopropylpyrazol-1yl)methanone

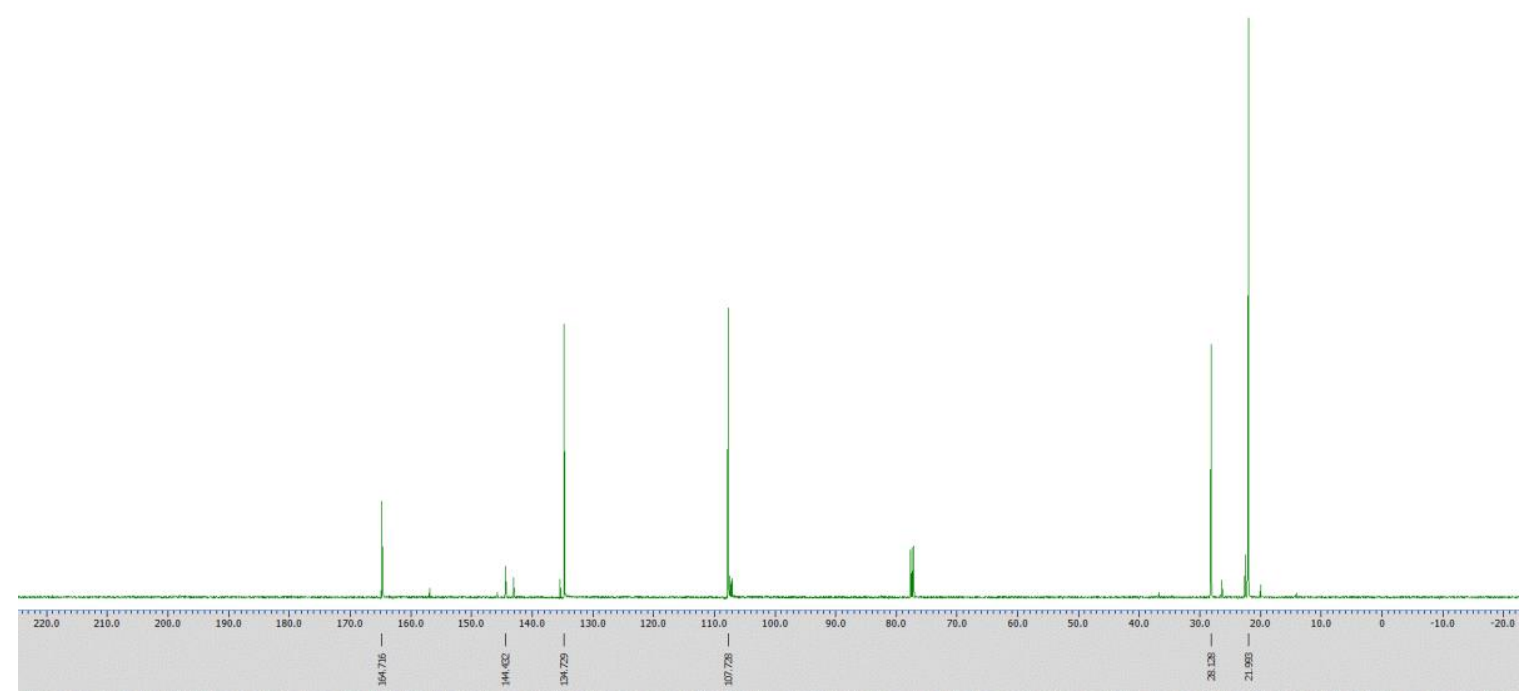

Figure $\mathrm{S} 2.9{ }^{13} \mathrm{C}-\mathrm{NMR}\left(101 \mathrm{MHz}, \mathrm{CDCl}_{3}\right)$ spectrum of bis(3-isopropylpyrazol-1yl)methanone 
<smiles>CC(C)(C(=O)OC(=O)OC(Cl)(Cl)C(Cl)(Cl)Cl)c1cc[nH]n1</smiles><smiles>CCCCCCCCO</smiles><smiles>CC(C)(C)Cc1ccn(C(=O)n2ccc(C(C)(C)C)n2)n1</smiles>

\section{bis(3-tert-butylpyrazol-1-yl)methanone}

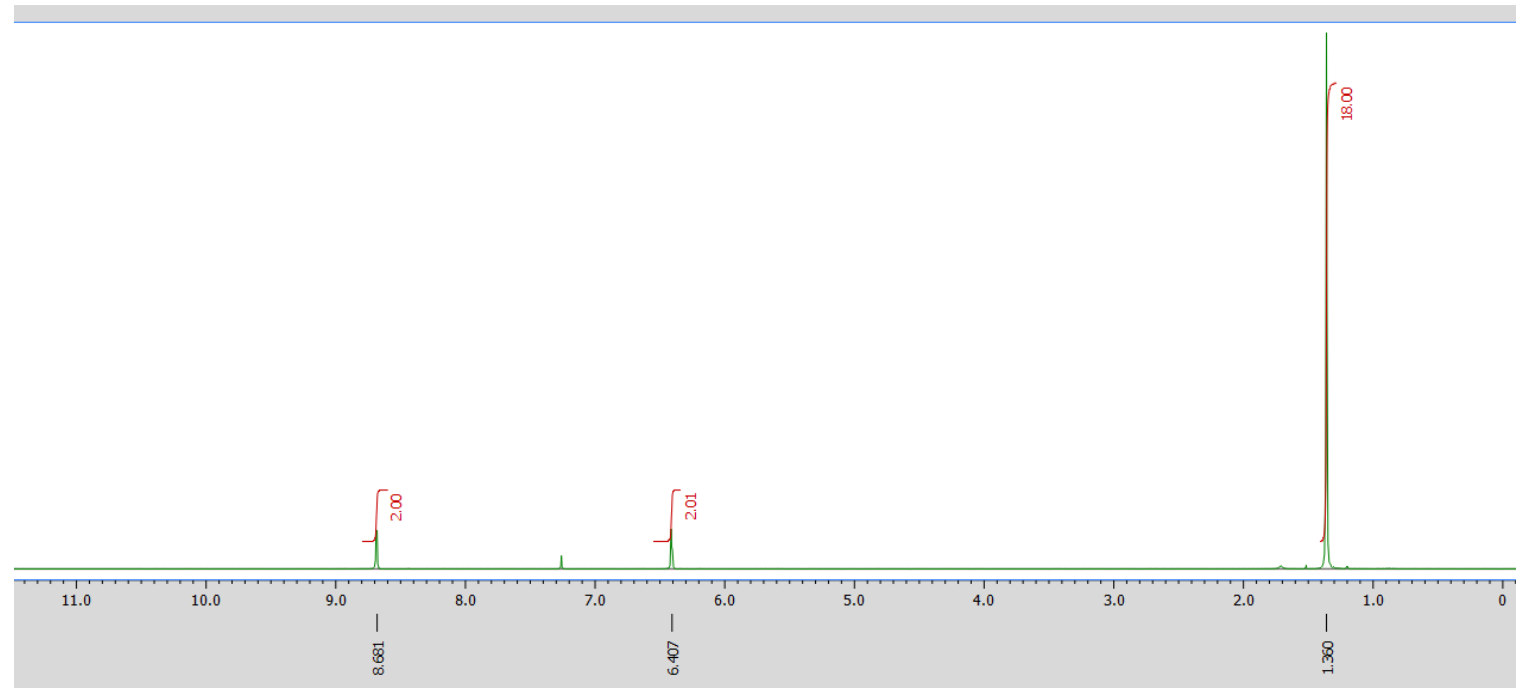

Figure $\mathrm{S} 2.10{ }^{1} \mathrm{H}$ NMR (400 $\left.\mathrm{MHz}, \mathrm{CDCl}_{3}\right)$ spectrum of bis(3-tert-butylpyrazol-1yl)methanone

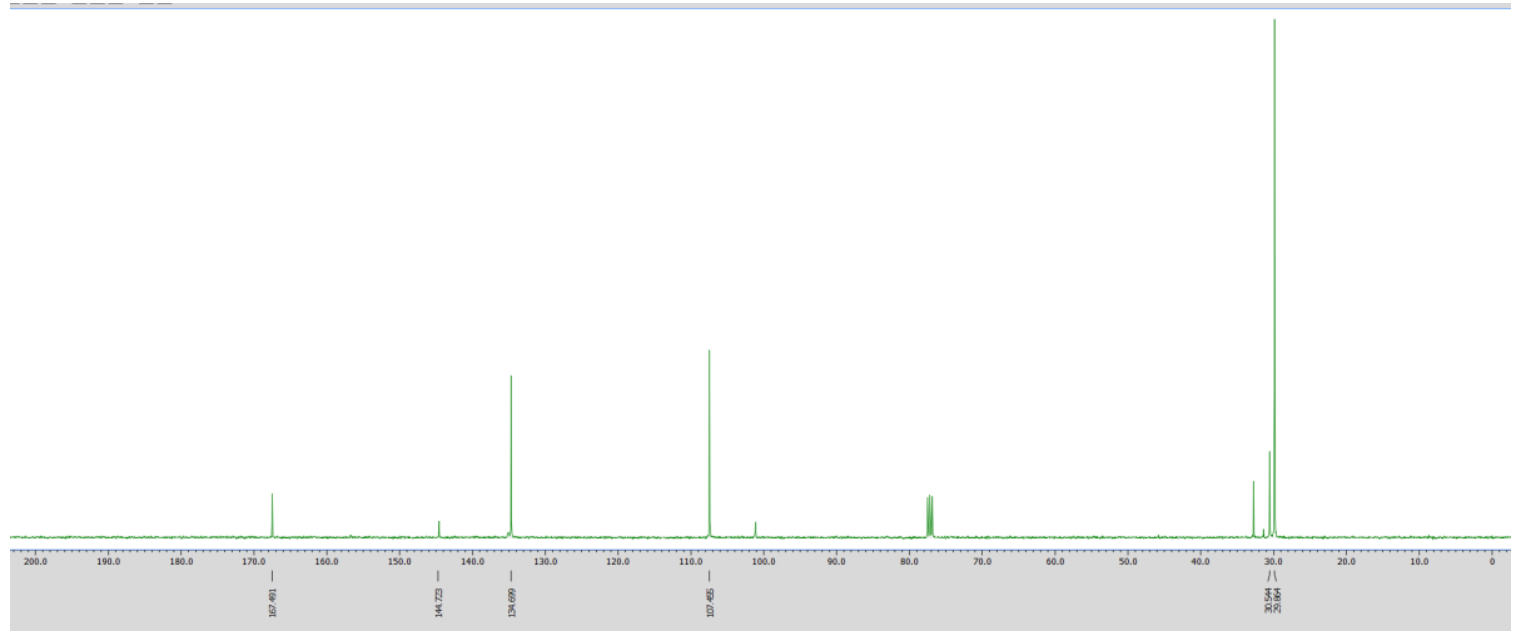


Figure $\mathrm{S} 2.11{ }^{13} \mathrm{C}-\mathrm{NMR}\left(101 \mathrm{MHz}, \mathrm{CDCl}_{3}\right)$ spectrum of bis(3-tert-butylpyrazol-1yl)methanone

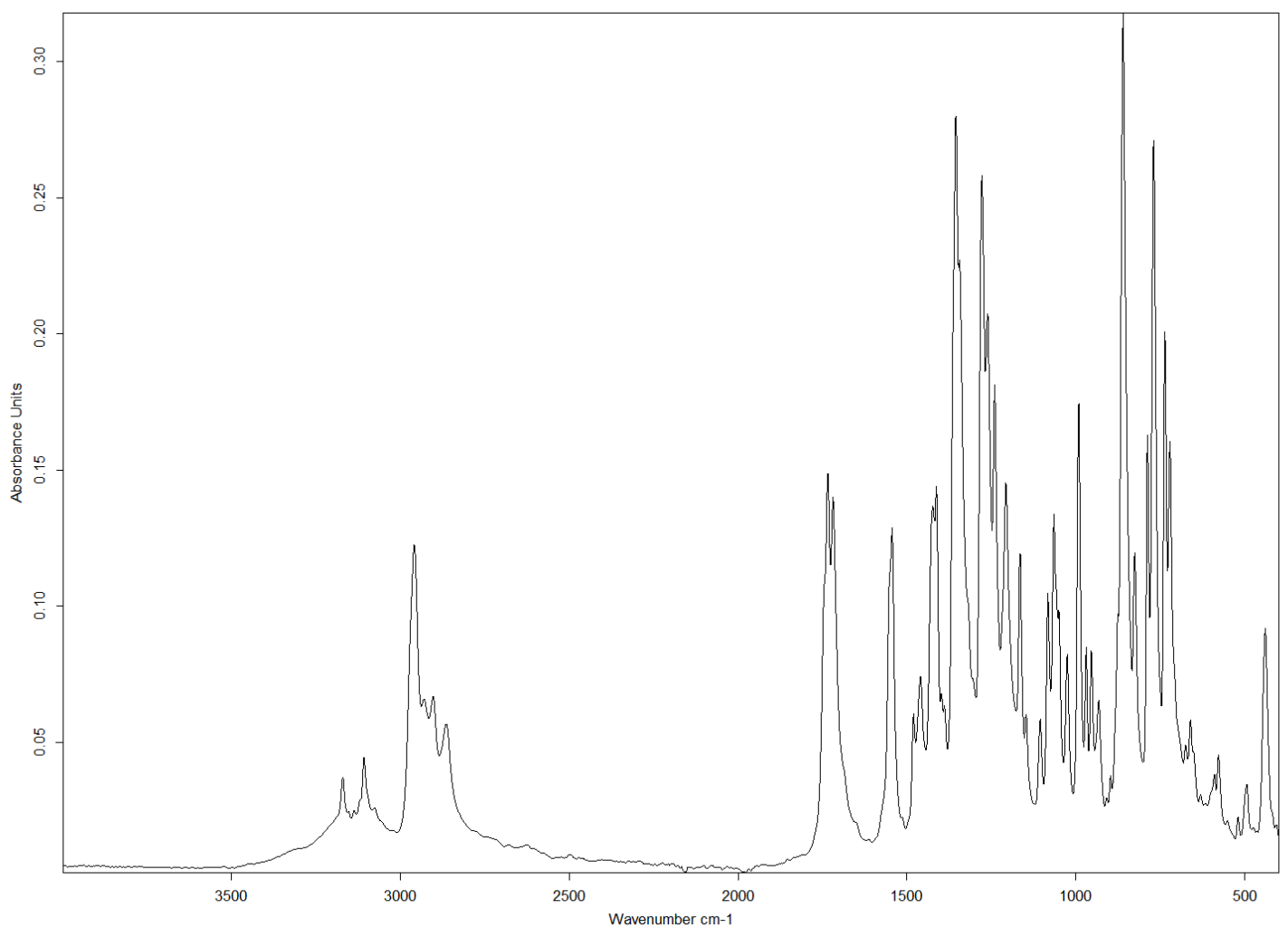

Figure S2.12 IR (Diamond ATR) spectrum of bis(3-tert-butylpyrazol-1-yl)methanone

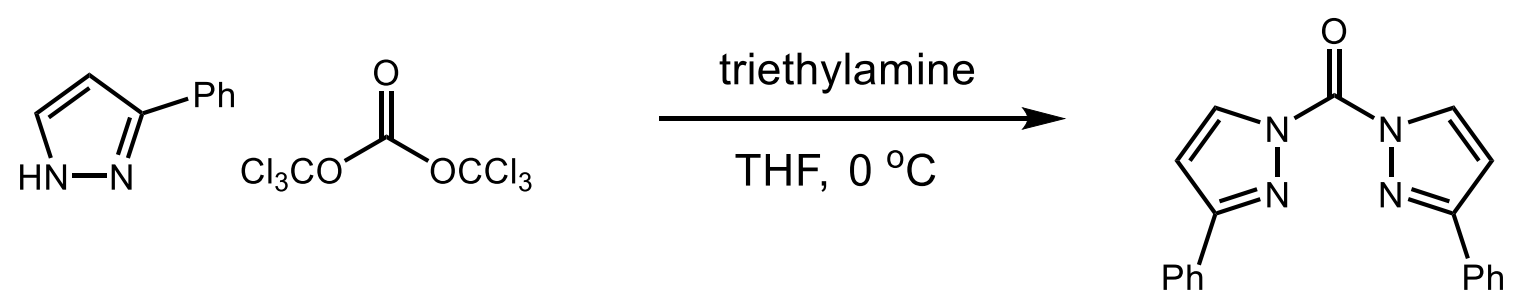

bis(3-phenylpyrazol-1-yl)methanone 


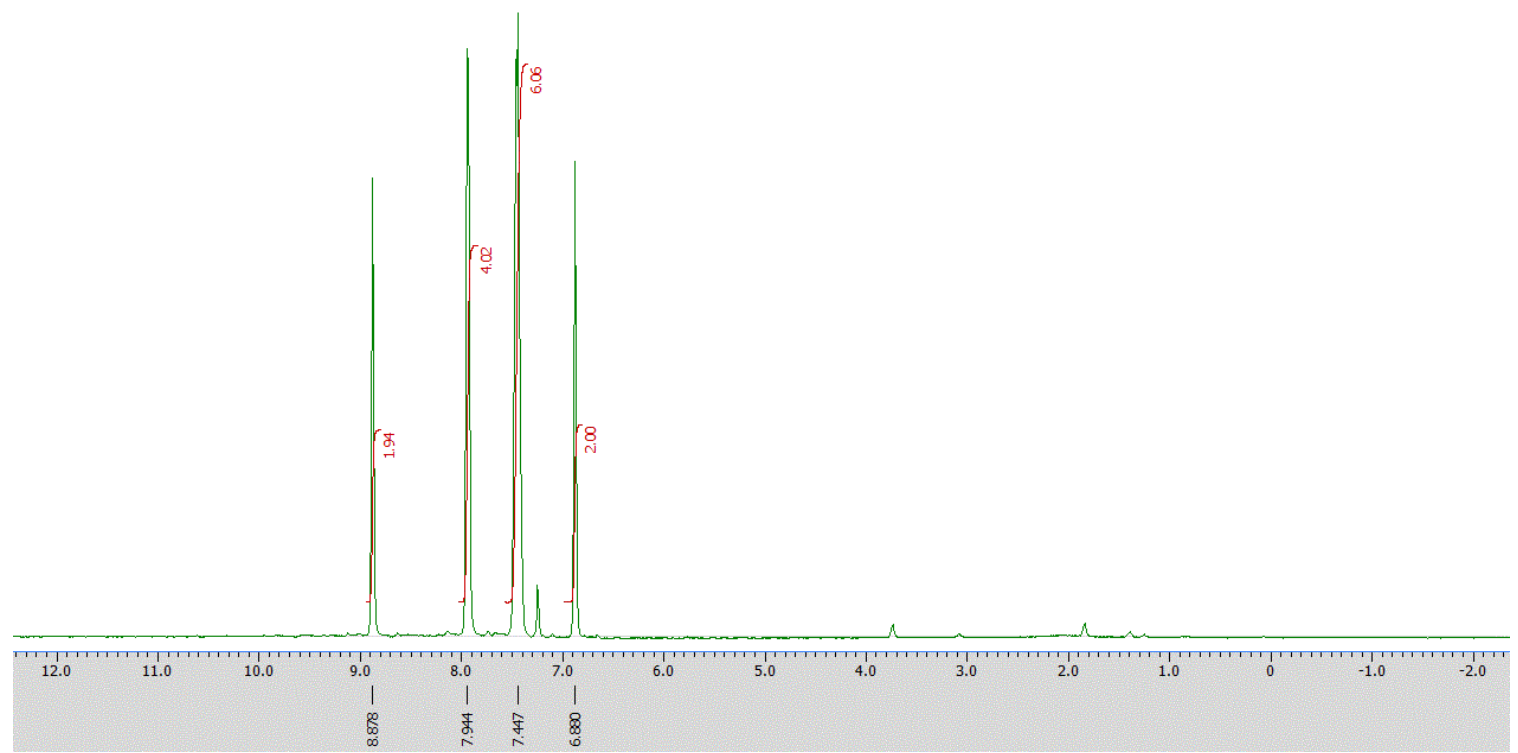

Figure $\mathrm{S} 2.13{ }^{1} \mathrm{H}$ NMR $\left(400 \mathrm{MHz}, \mathrm{CDCl}_{3}\right)$ spectrum of bis(3-phenylpyrazol-1yl)methanone

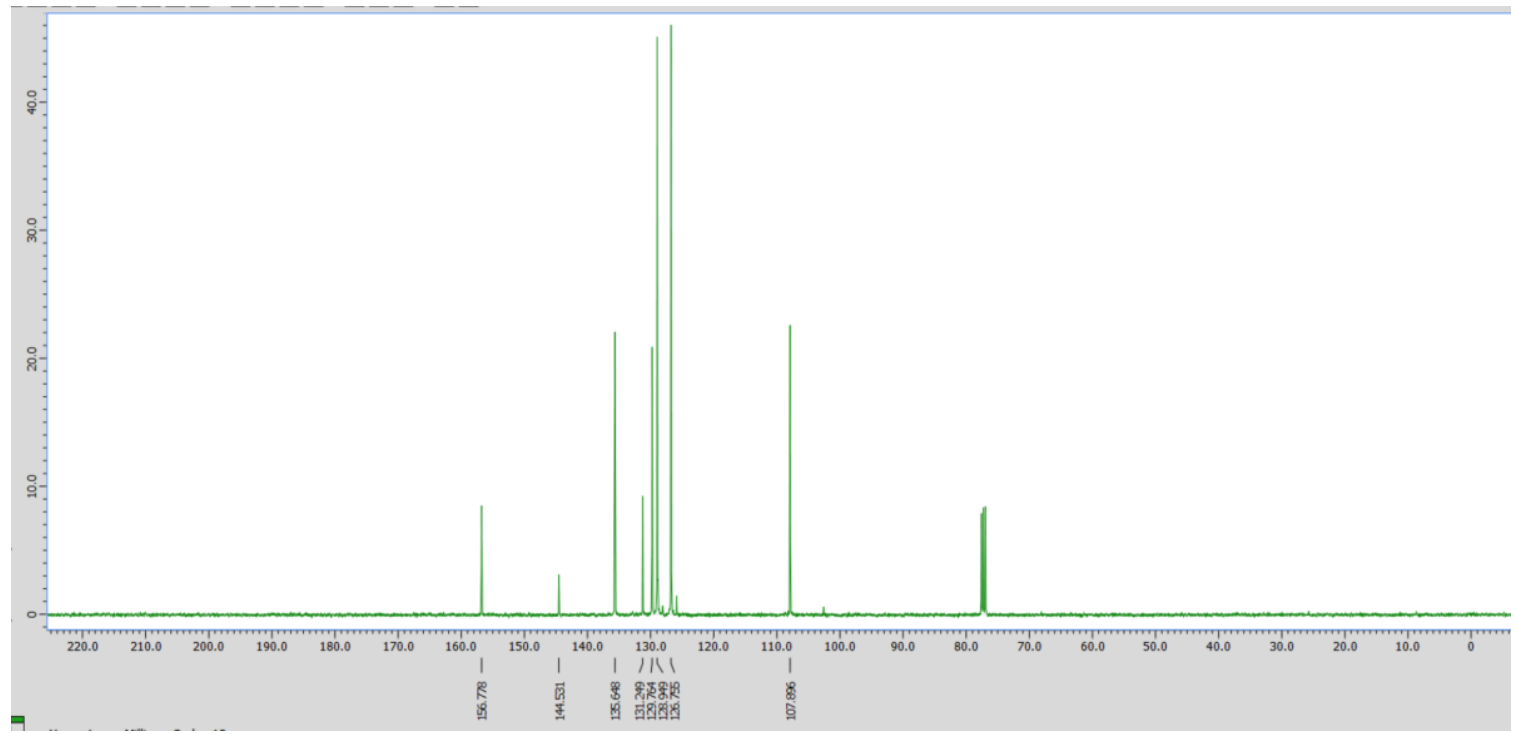

Figure $\mathrm{S} 2.14{ }^{13} \mathrm{C} \quad \mathrm{NMR}\left(101 \mathrm{MHz}, \mathrm{CDCl}_{3}\right)$ spectrum of bis(3-phenylpyrazol-1yl)methanone 


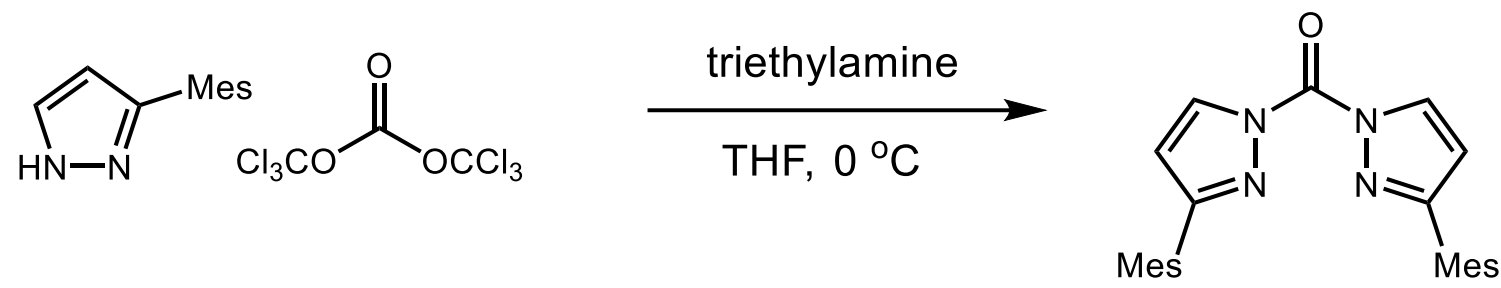

bis(3-mesitylpyrazol-1-yl)methanone.

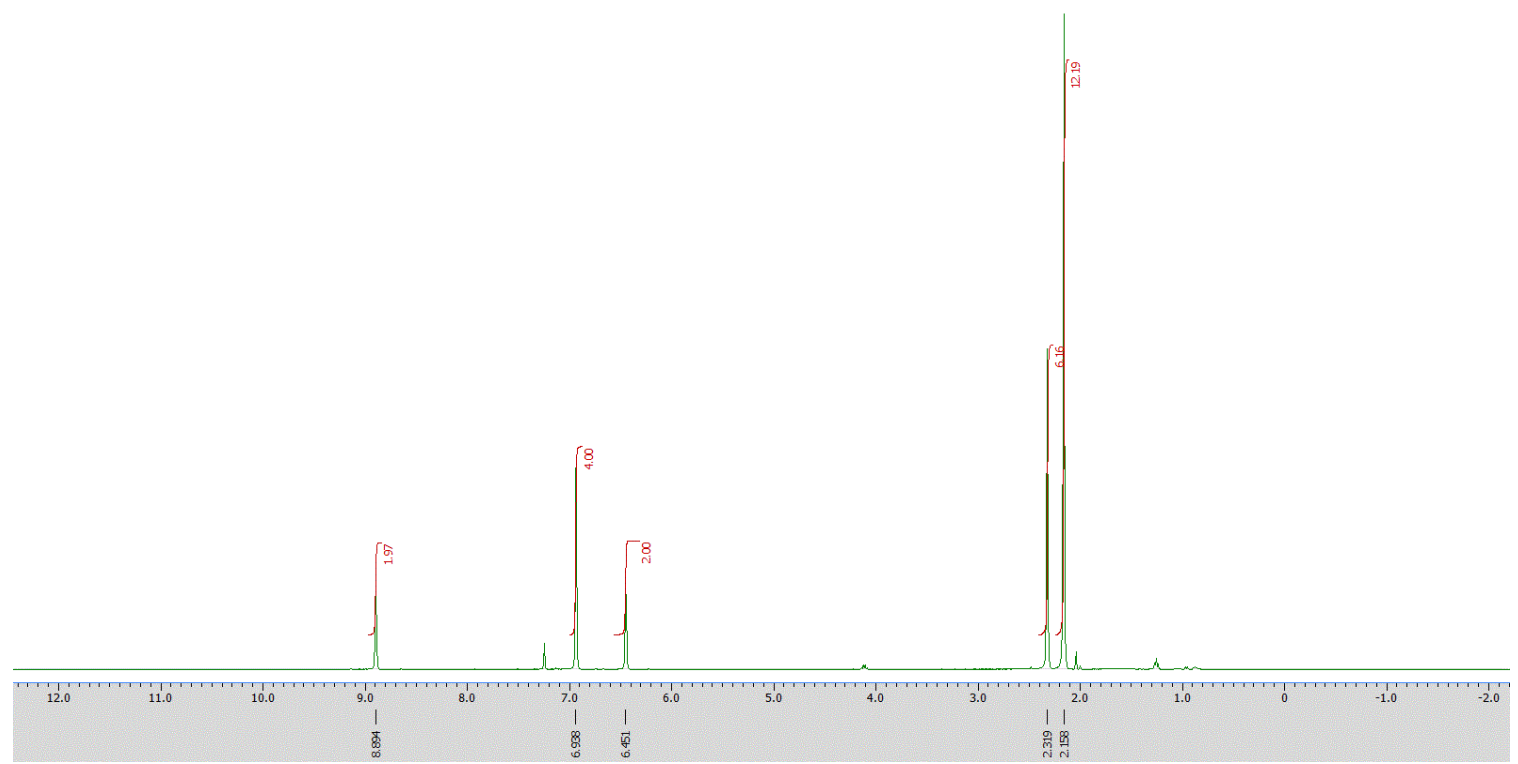

Figure $\mathrm{S} 2.15{ }^{1} \mathrm{H}$ NMR (400 $\left.\mathrm{MHz}, \mathrm{CDCl}_{3}\right)$ spectrum of bis(3-mesitylpyrazol-1yl)methanone

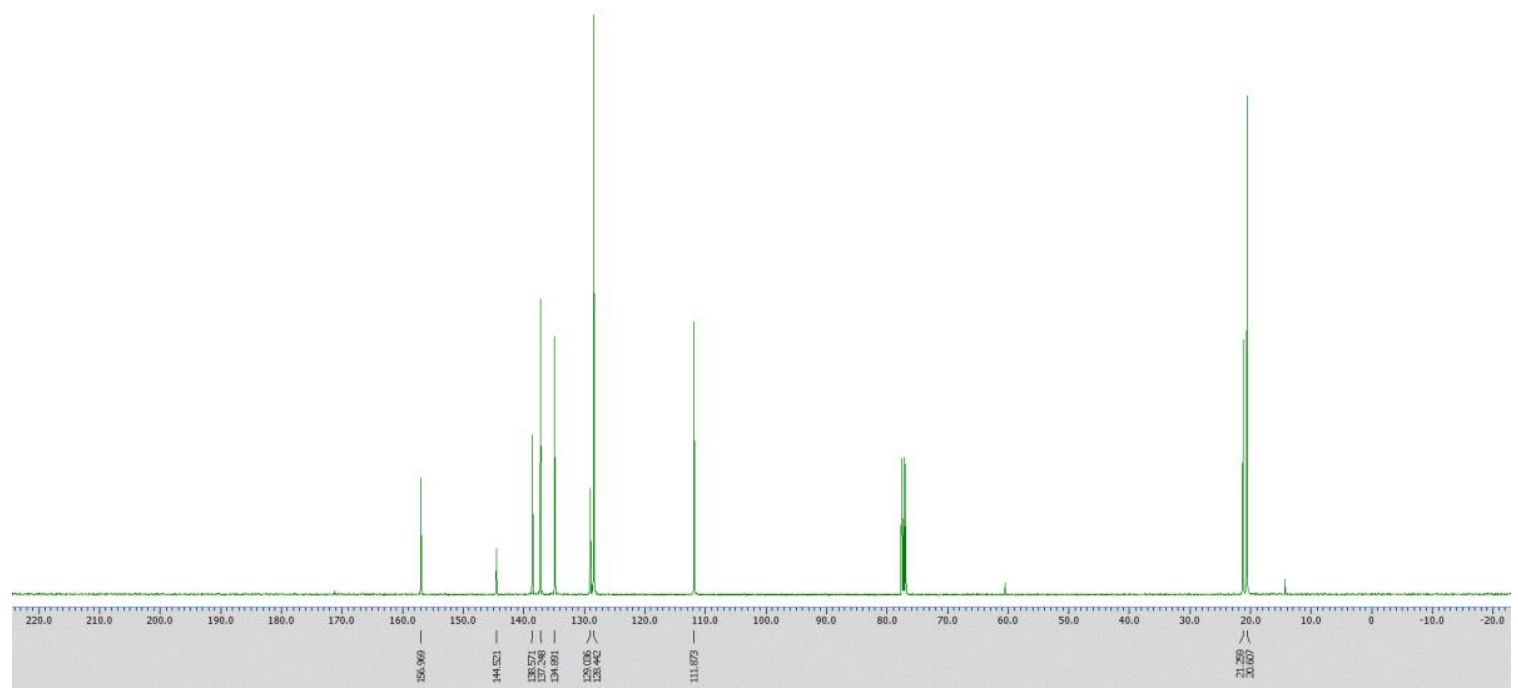


Figure $\mathrm{S} 2.16{ }^{13} \mathrm{C}$ NMR (101 $\left.\mathrm{MHz}, \mathrm{CDCl}_{3}\right)$ spectrum of bis(3-mesitylpyrazol-1yl)methanone

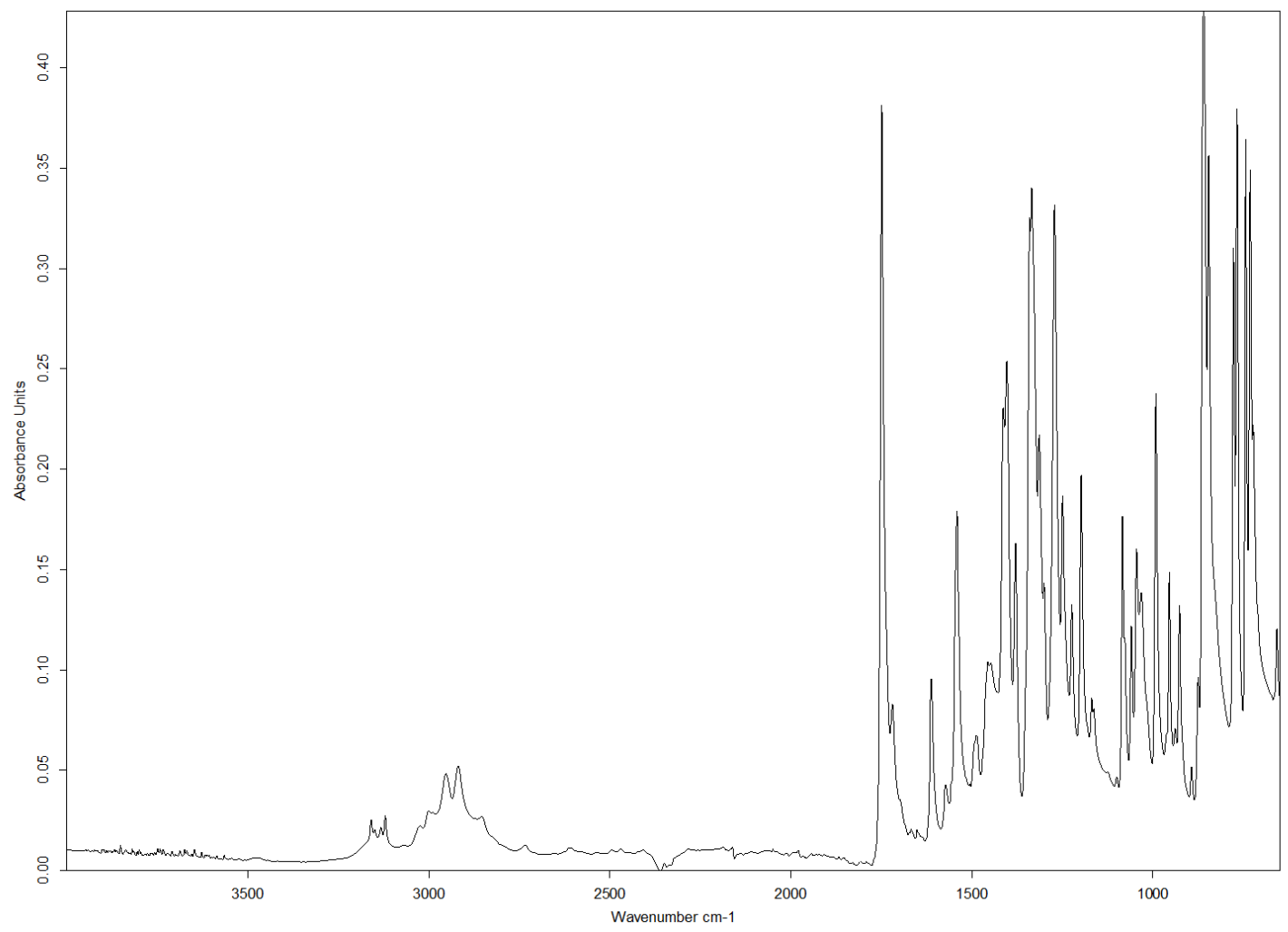

Figure S2.17 IR (Diamond ATR) spectrum of bis(3-mesitylpyrazol-1-yl)methanone

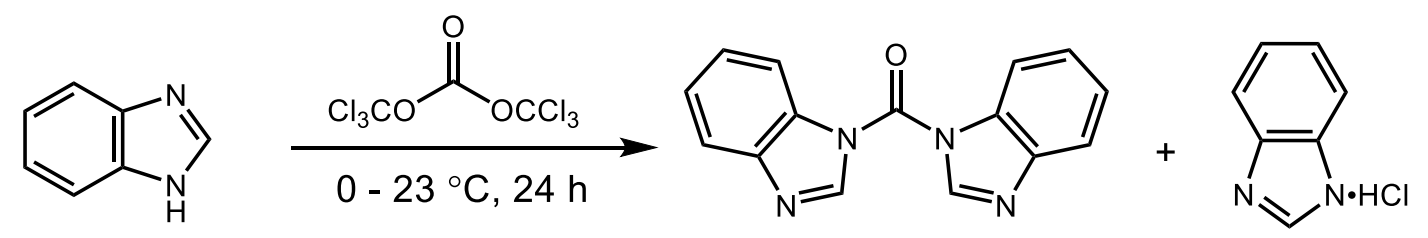

bis(benzimidazol-1-yl)methanone 


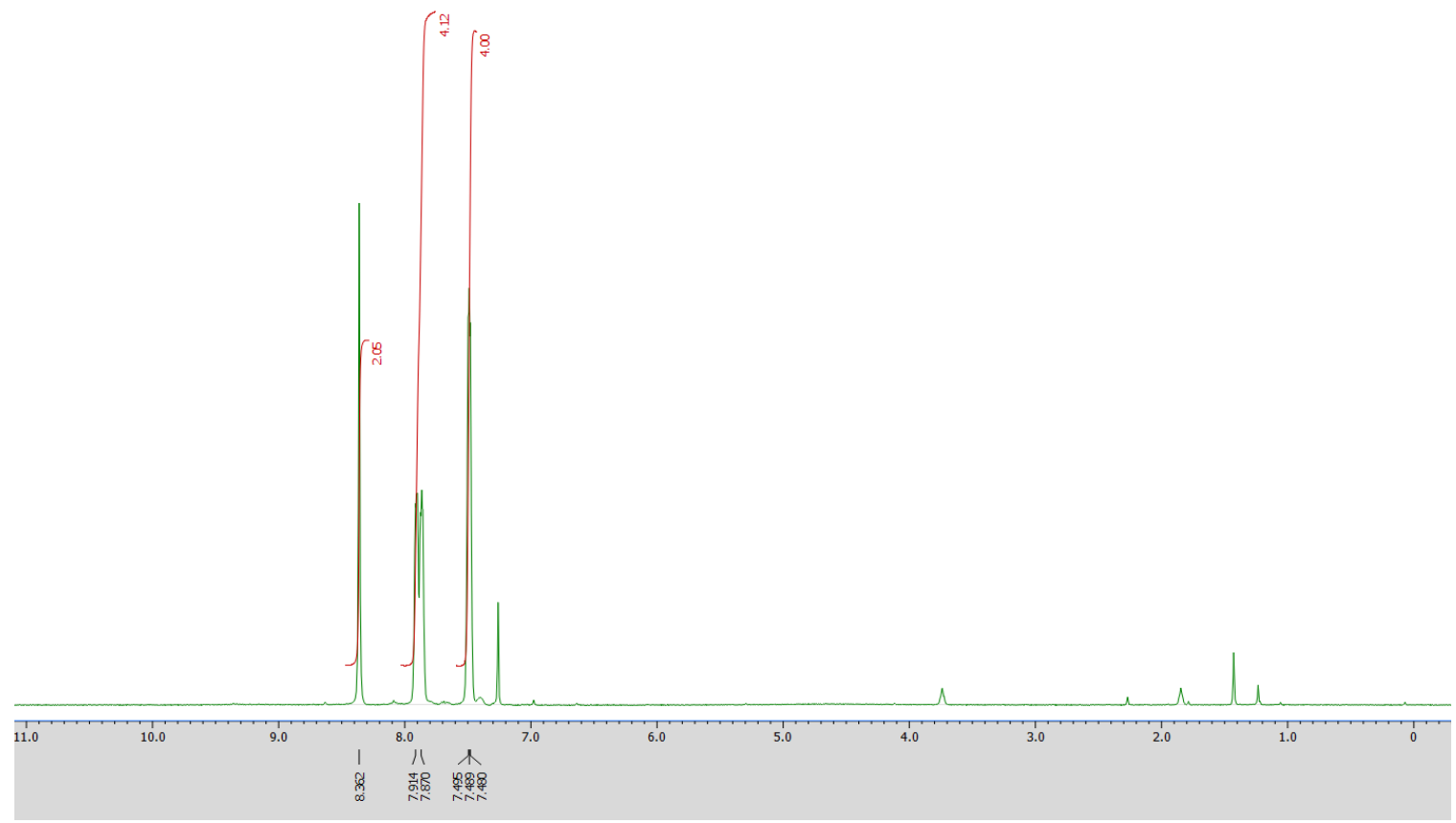

Figure $\mathrm{S} 2.18{ }^{1} \mathrm{H}-\mathrm{NMR}\left(400 \mathrm{MHz}, \mathrm{CDCl}_{3}\right.$ ) spectrum of bis(benzimidazol-1-yl)methanone

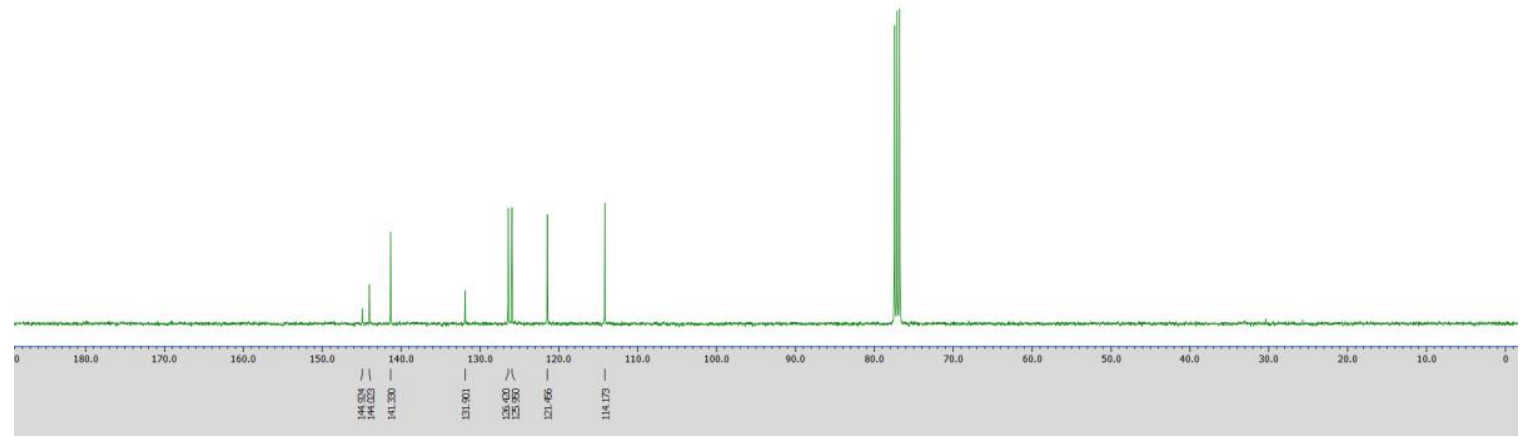

Figure S2.19 ${ }^{13} \mathrm{C}-\mathrm{NMR} \quad\left(100 \mathrm{MHz}, \mathrm{CDCl}_{3}\right)$ spectrum of bis(benzimidazol-1yl)methanone 
<smiles>Cc1nc2ccccc2[nH]1</smiles><smiles>Cc1nc2ccccc2n1C(=O)n1c(C)nc2ccccc21</smiles>

bis(2-methylbenzimidazol-1-yl)methanone.

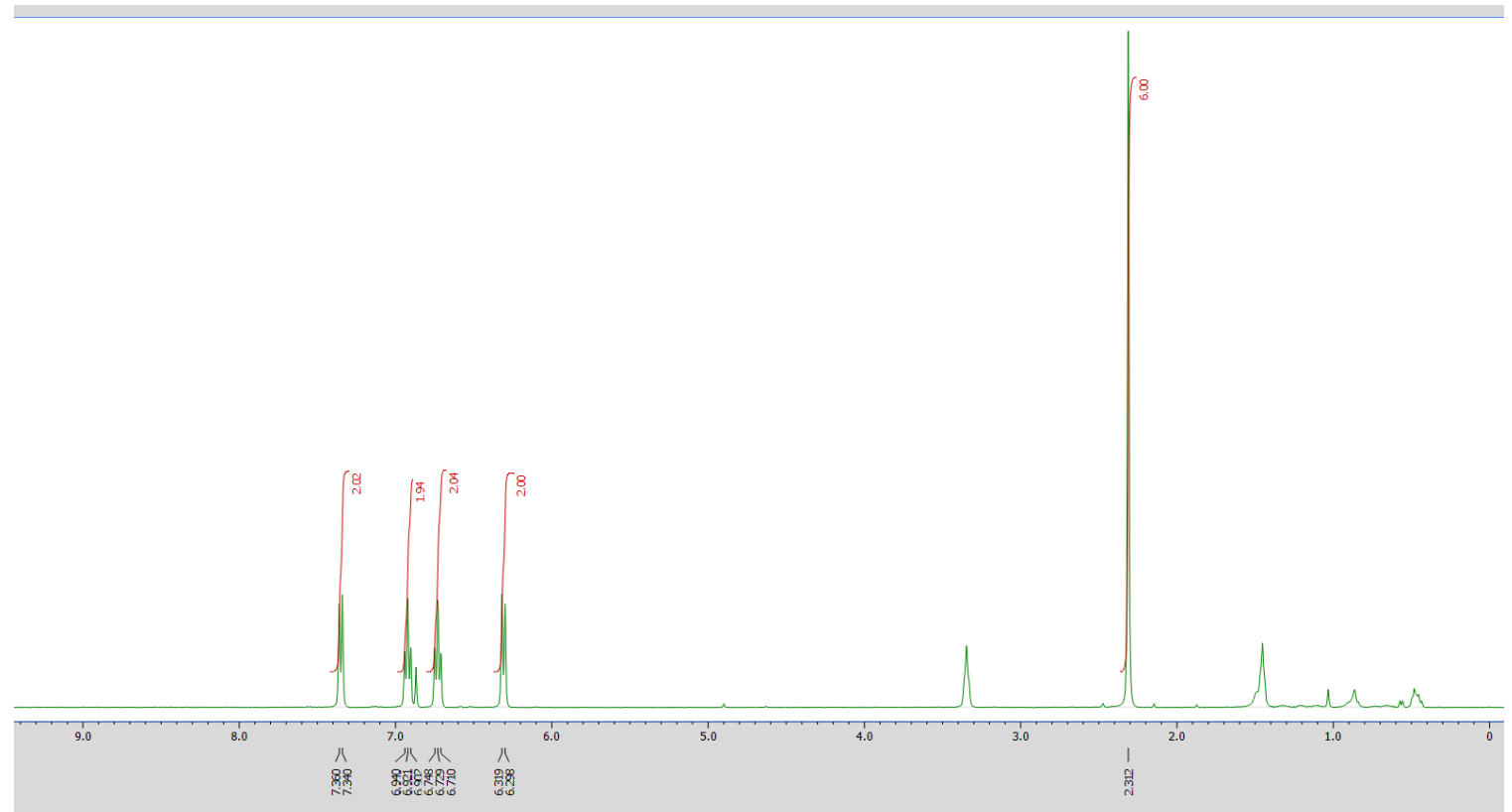

Figure $\mathrm{S} 2.20{ }^{1} \mathrm{H}-\mathrm{NMR}\left(400 \mathrm{MHz}, \mathrm{CDCl}_{3}\right)$ spectrum of bis(2-methylbenzimidazol-1yl)methanone

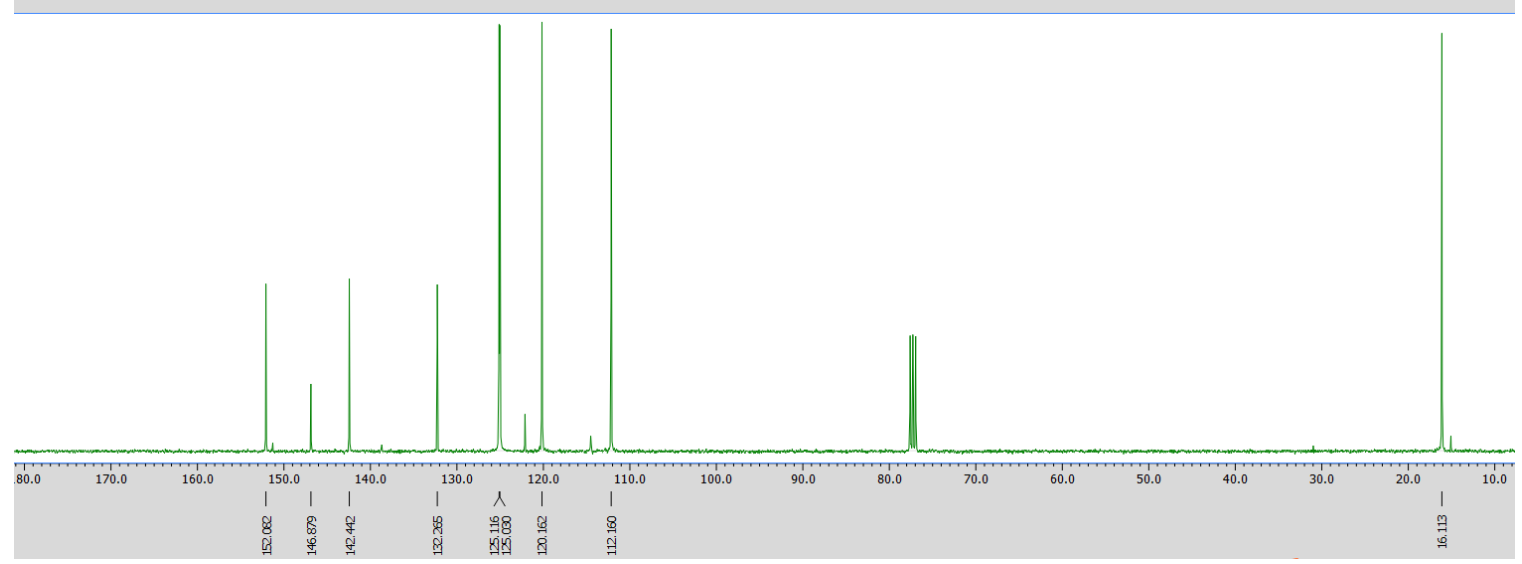


Figure S2.21 ${ }^{13} \mathrm{C}-\mathrm{NMR}\left(100 \mathrm{MHz}, \mathrm{CDCl}_{3}\right)$ spectrum of bis(2-methylbenzimidazol-1yl)methanone 
3. Spectral Data for Bis(pyrazolyl)alkanes
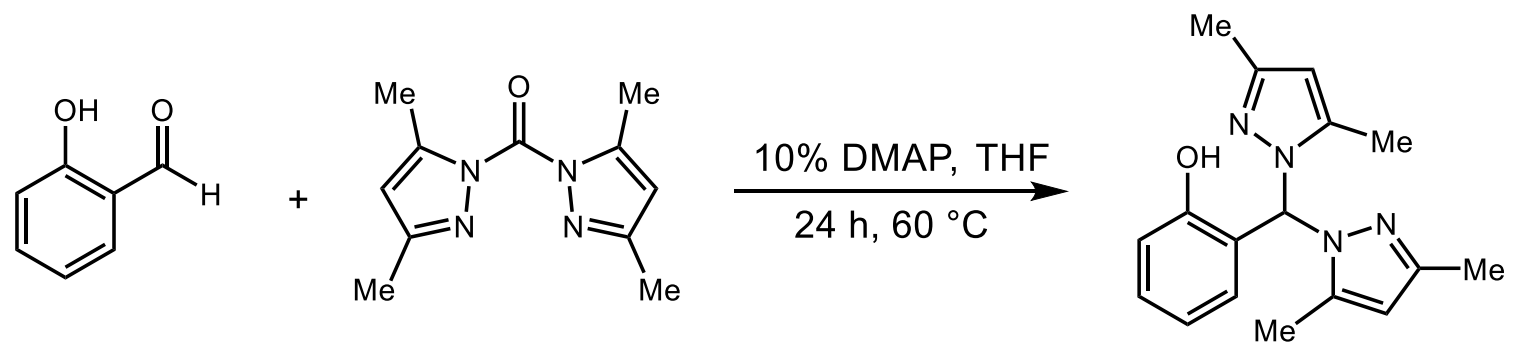

2-bis(3,5-dimethylpyrazol-1-yl)methylphenol (3)

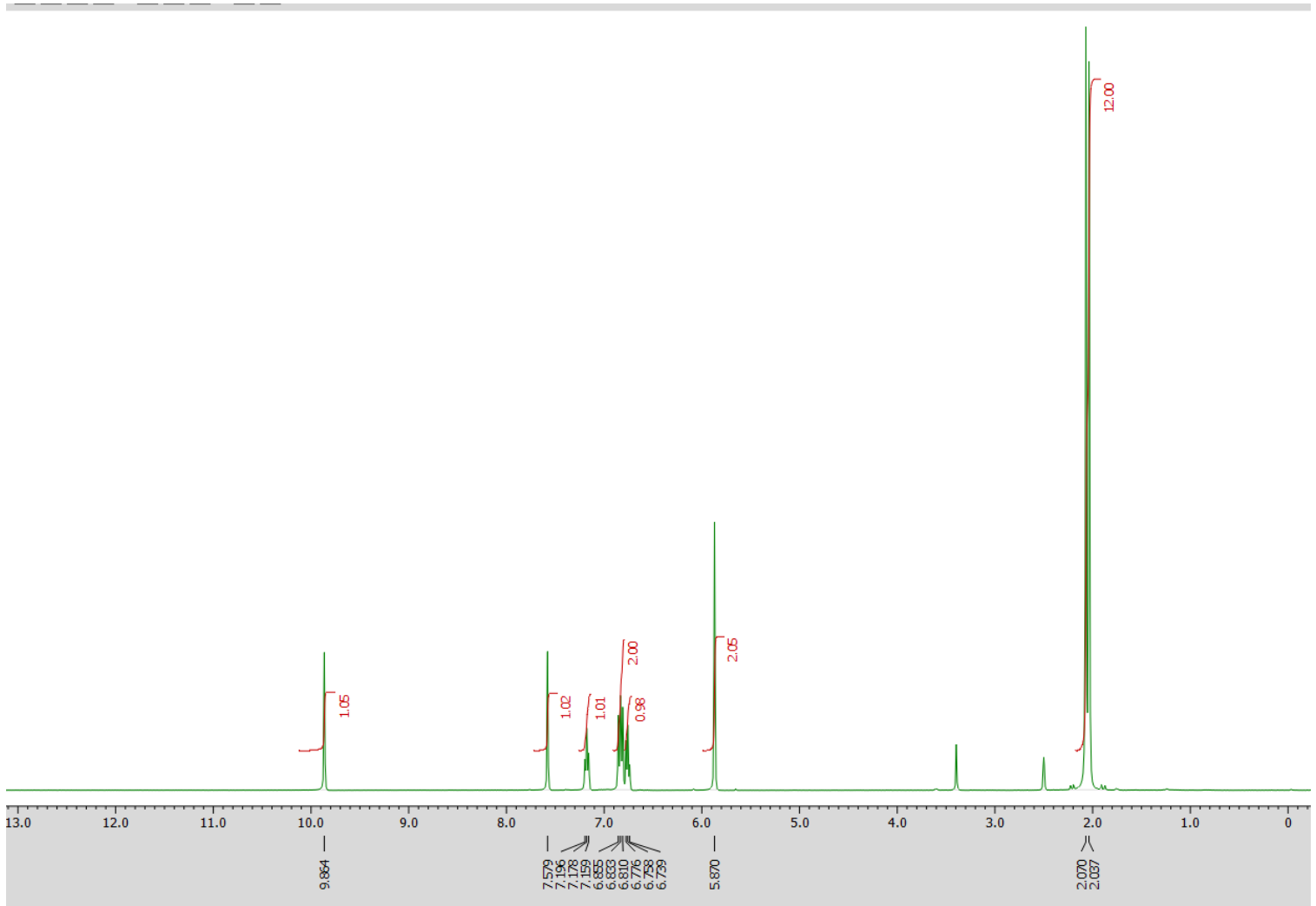

Figure S3.1 ${ }^{1} \mathrm{H}$ NMR (400 MHz, DMSO-d6) spectrum of 2-bis(3,5-dimethylpyrazol-1yl)methylphenol (3) 


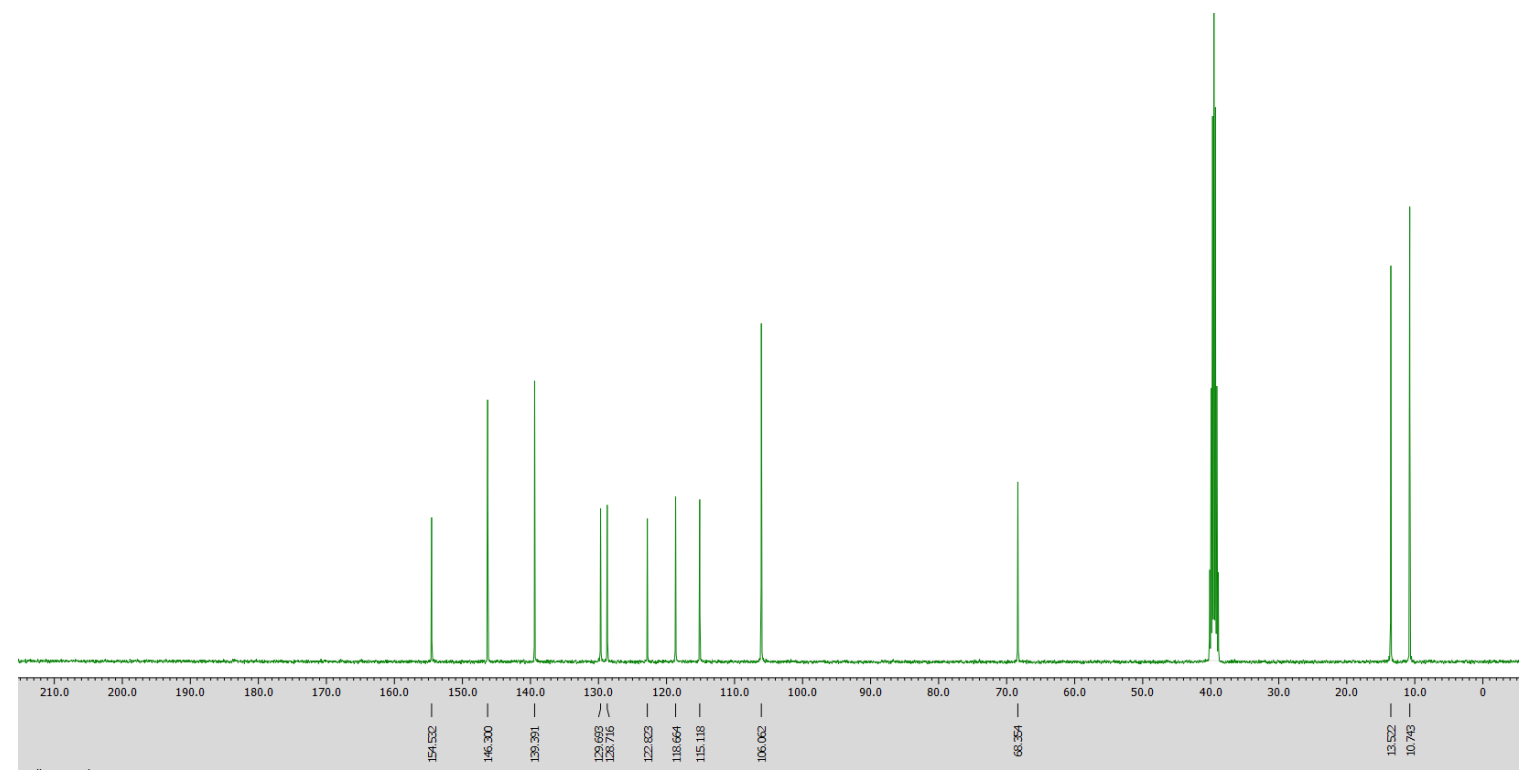

Figure S3.2 ${ }^{13} \mathrm{C}$ NMR (100 MHz, DMSO-d6) spectrum of 2-bis(3,5-dimethylpyrazol-1yl)methylphenol (3)

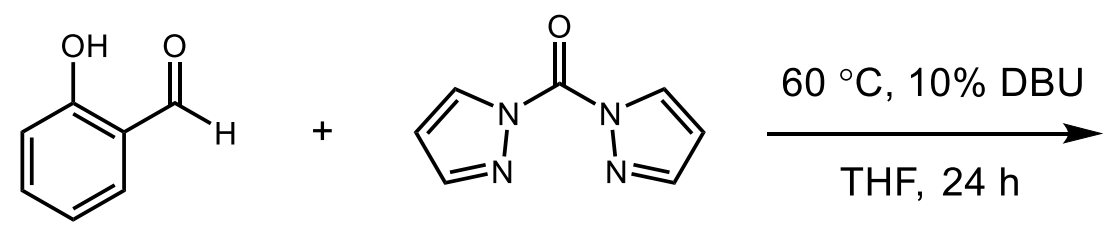<smiles>Oc1ccccc1C(n1cccn1)n1cccn1</smiles>

\section{2-bis(pyrazol-1-yl)methylphenol.}




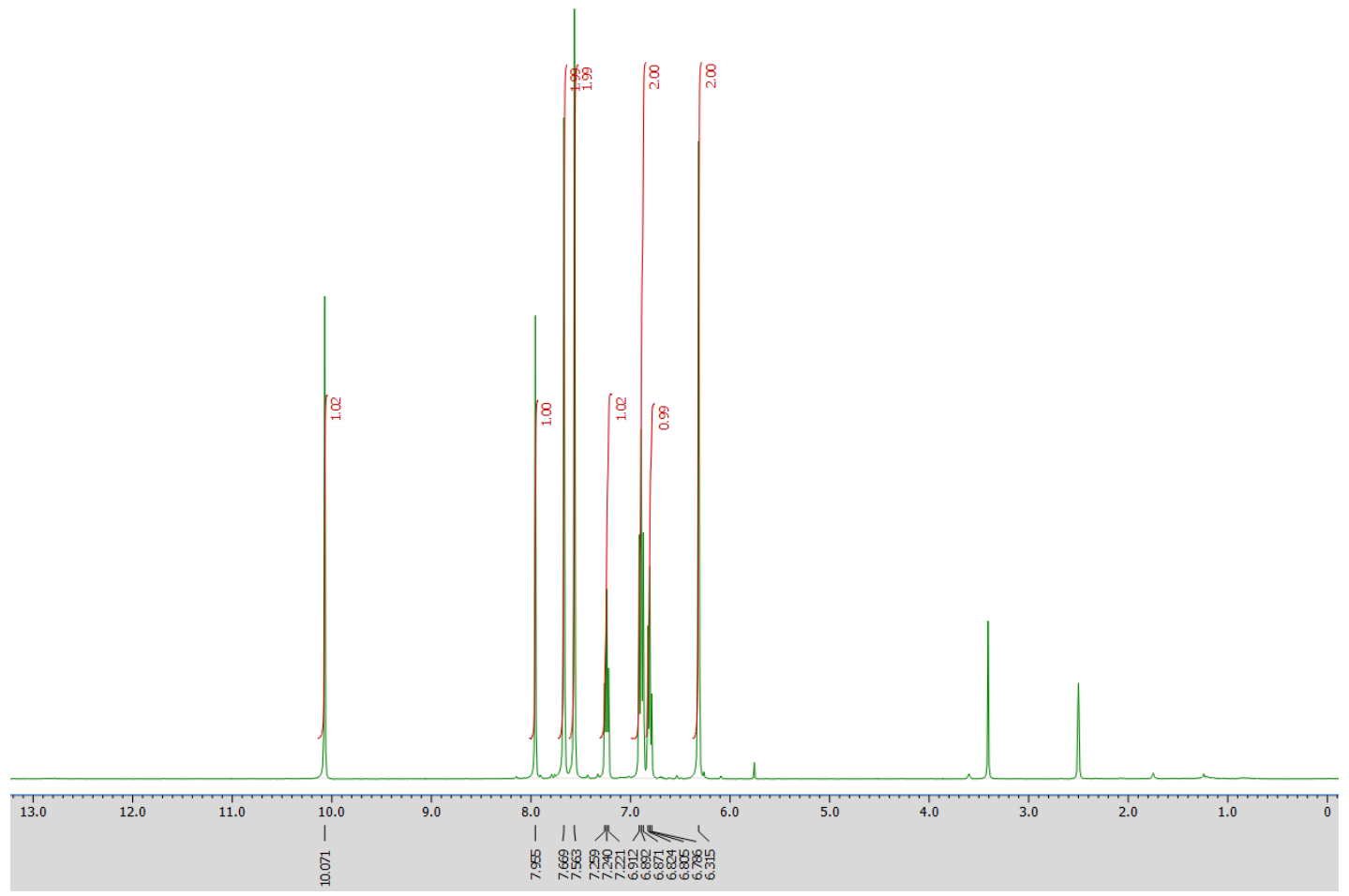

Figure S3.3 ${ }^{1} \mathrm{H} \quad \mathrm{NMR} \quad(400 \quad \mathrm{MHz}$, DMSO-d6) spectrum of 2-bis(pyrazol-1yl)methylphenol

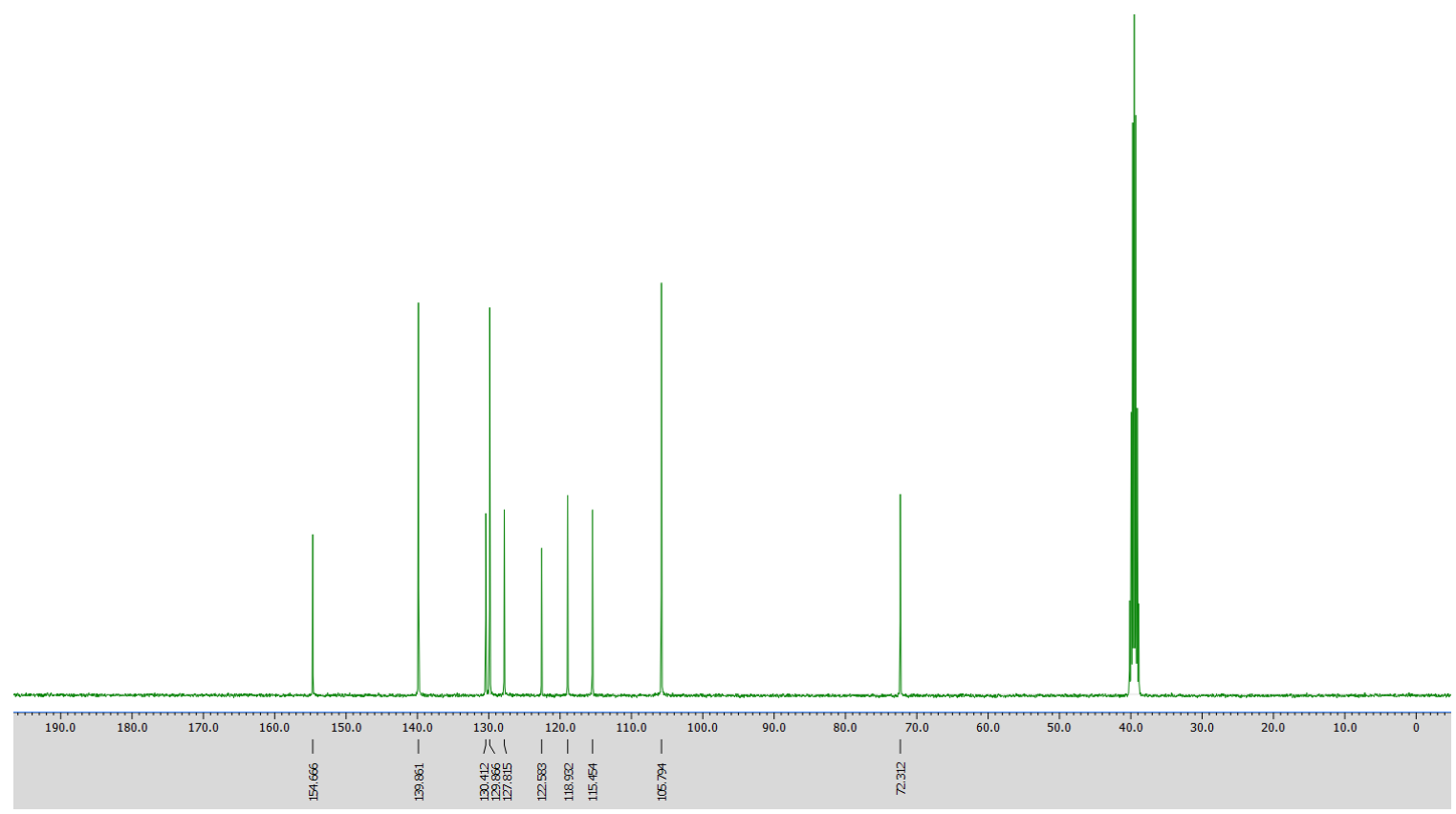


Figure $\quad \mathrm{S} 3.4{ }^{13} \mathrm{C} \quad \mathrm{NMR} \quad(100 \mathrm{MHz}, \quad$ DMSO-d6) spectrum of 2-bis(pyrazol-1yl)methylphenol<smiles>CCc1cc(CC)n(C(=O)n2nc(CC)cc2CC)n1</smiles><smiles>CCc1cc(CC)n(C(c2ccccc2O)n2nc(CC)cc2CC)n1</smiles>

2-bis(3,5-dimethylpyrazol-1-yl)methylphenol.

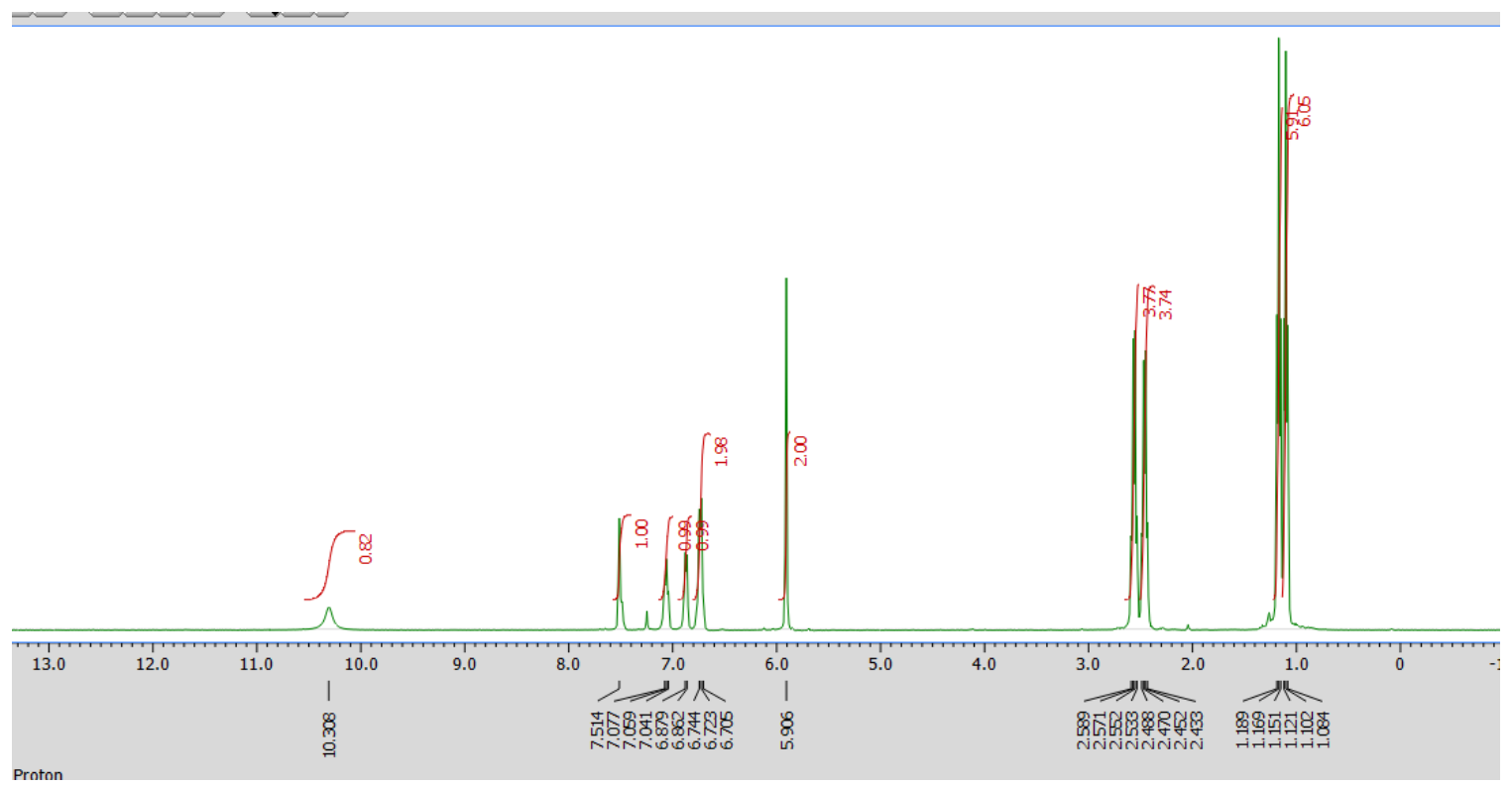

Figure $\mathrm{S} 3.5{ }^{1} \mathrm{H}$ NMR (400 MHz, $\mathrm{CDCl}_{3}$ ) spectrum of 2-bis(3,5-diethylpyrazol-1yl)methylphenol 


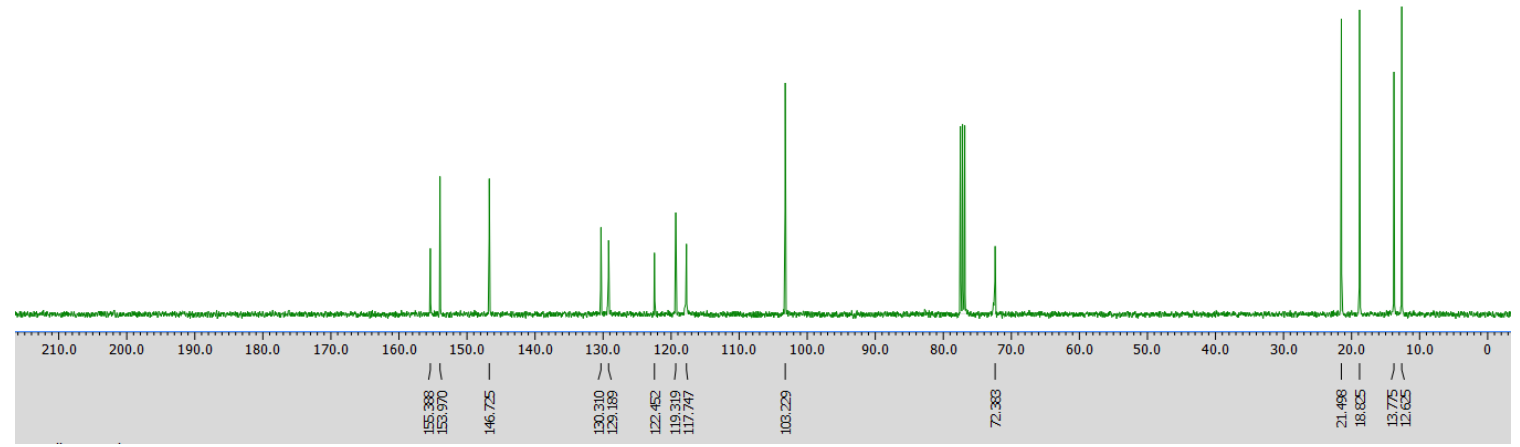

Figure $\mathrm{S} 3.6{ }^{13} \mathrm{C}$ NMR (101 $\left.\mathrm{MHz}, \mathrm{CDCl}_{3}\right)$ spectrum of 2-bis(3,5-diethylpyrazol-1yl)methylphenol

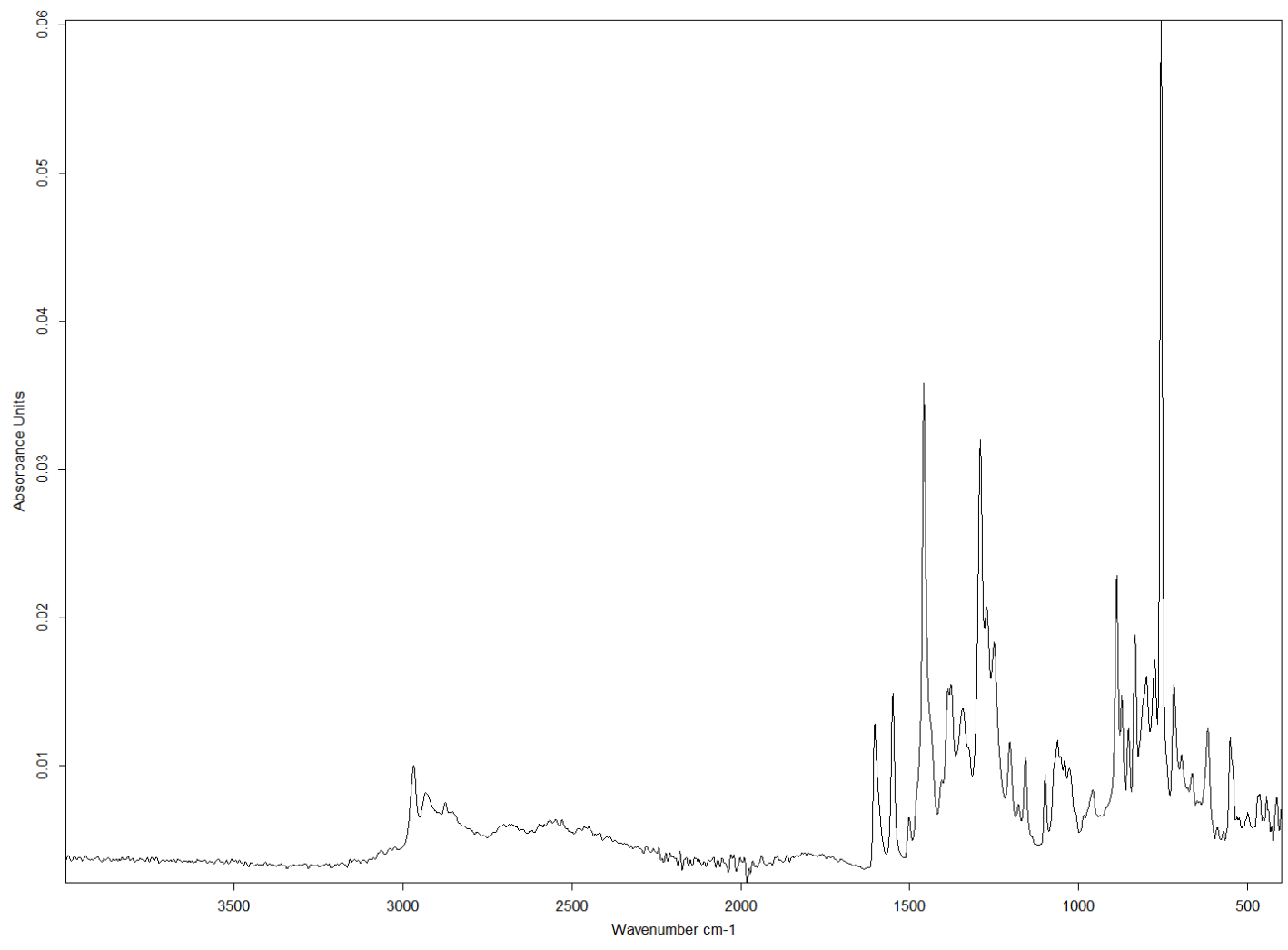

Figure S3.7 IR spectrum of 2-bis(3,5-diethylpyrazol-1-yl)methylphenol 


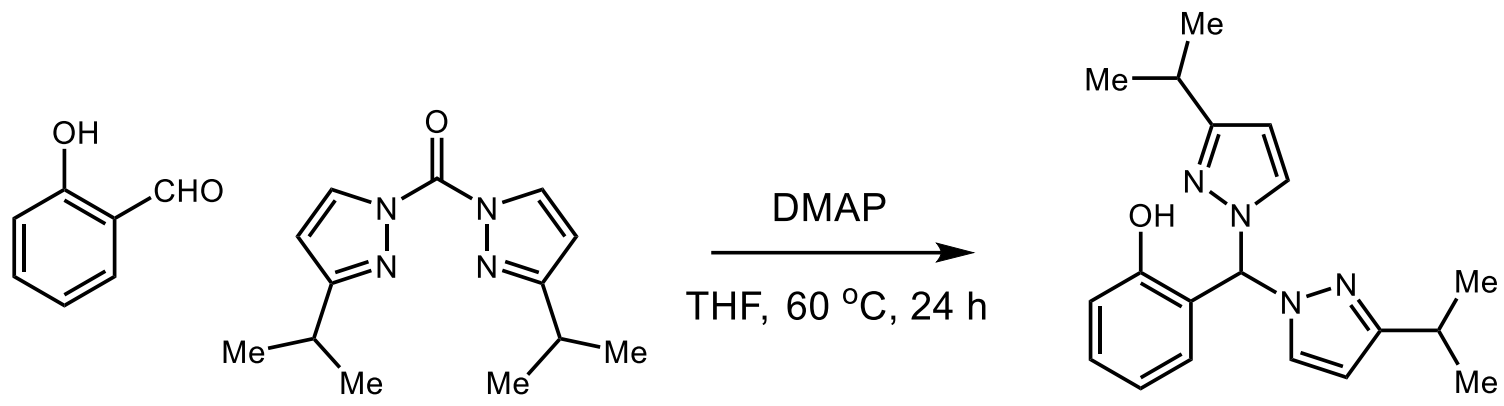

2-bis(3-isopropylpyrazol-1-yl)methylphenol.

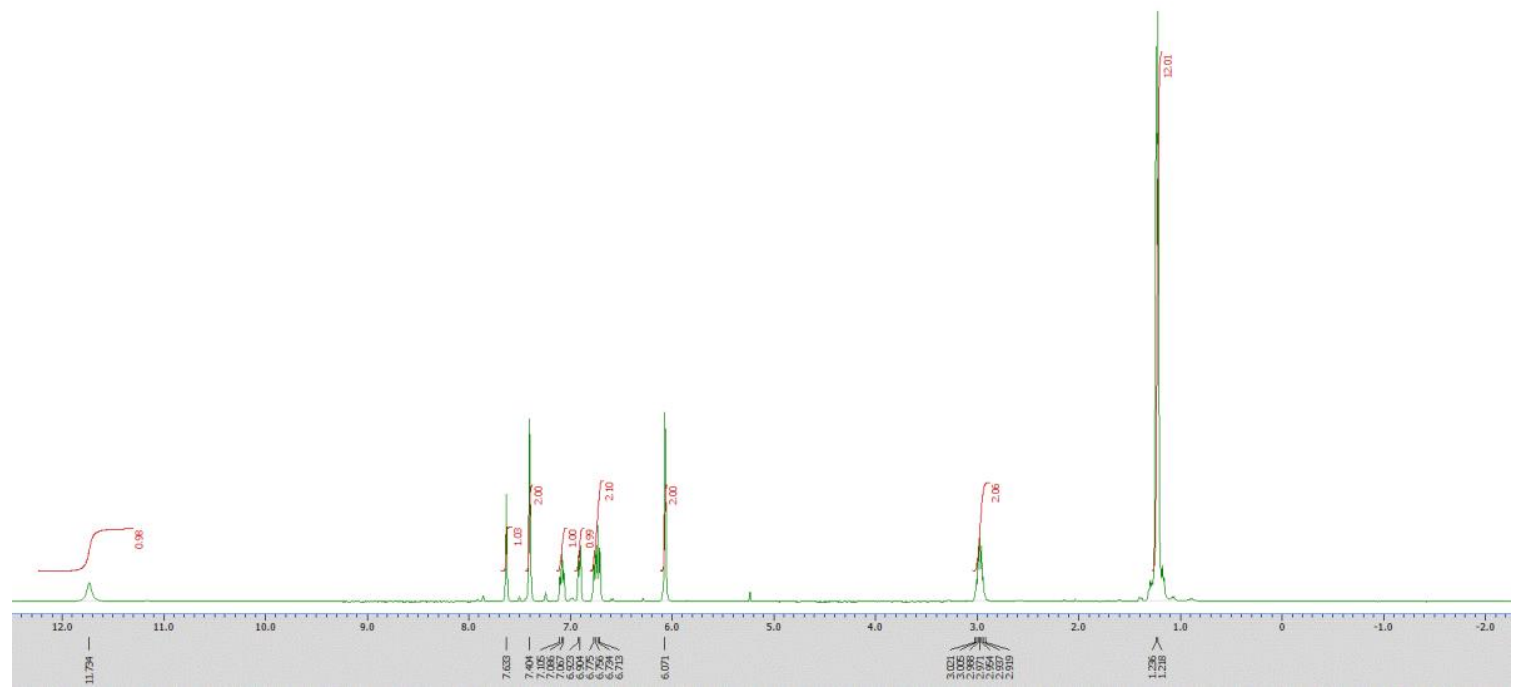

Figure $\mathrm{S} 3.8{ }^{1} \mathrm{H}$ NMR (400 $\left.\mathrm{MHz}, \mathrm{CDCl}_{3}\right)$ spectrum of 2-bis(3-isopropylpyrazol-1yl)methylphenol 


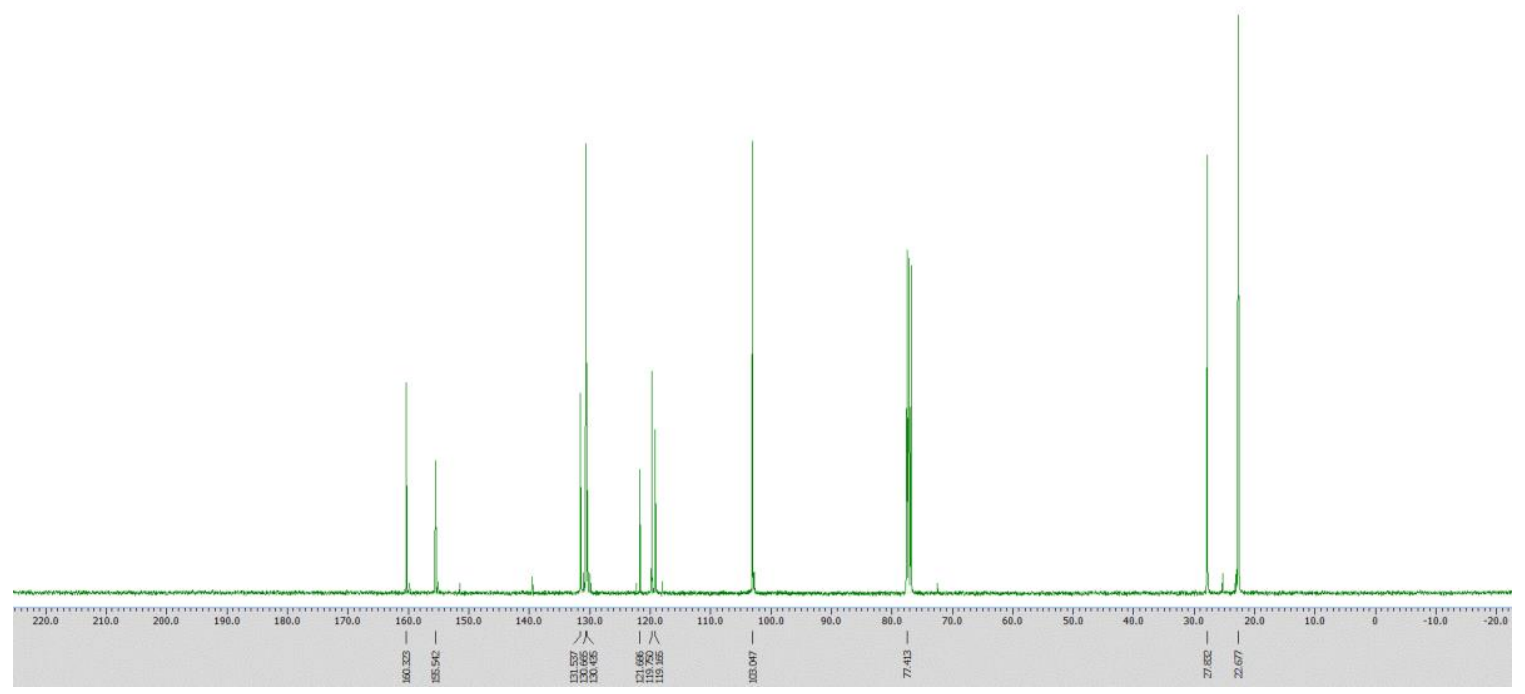

Figure $\mathrm{S} 3.9{ }^{13} \mathrm{C} \mathrm{NMR}\left(101 \mathrm{MHz}, \mathrm{CDCl}_{3}\right)$ spectrum of 2-bis(3-isopropylpyrazol-1-

\section{yl)methylphenol}<smiles>O=Cc1ccccc1O</smiles><smiles>O=C(n1ccc(Br)n1)n1ccc(Br)n1</smiles>

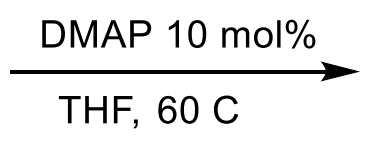<smiles>CC(C)(C)c1ccn(C(c2ccccc2O)n2ccc(C(C)(Br)Br)n2)n1</smiles>

\section{2-bis(3-tert-butylpyrazol-1-yl)methylphenol.}




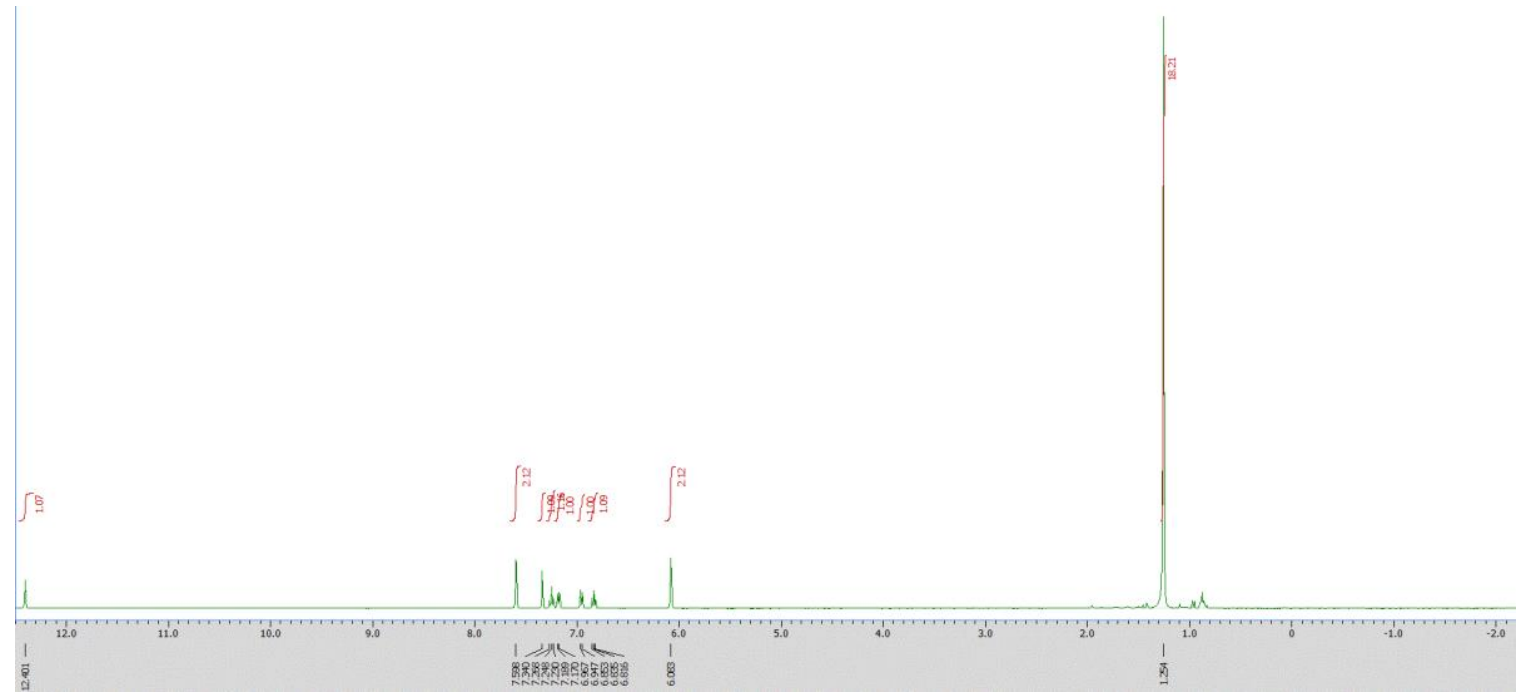

Figure $\mathrm{S} 3.10{ }^{1} \mathrm{H}$ NMR $\left(400 \mathrm{MHz}, \mathrm{CDCl}_{3}\right)$ spectrum of 2-bis(3-tert-butylpyrazol-1yl)methylphenol

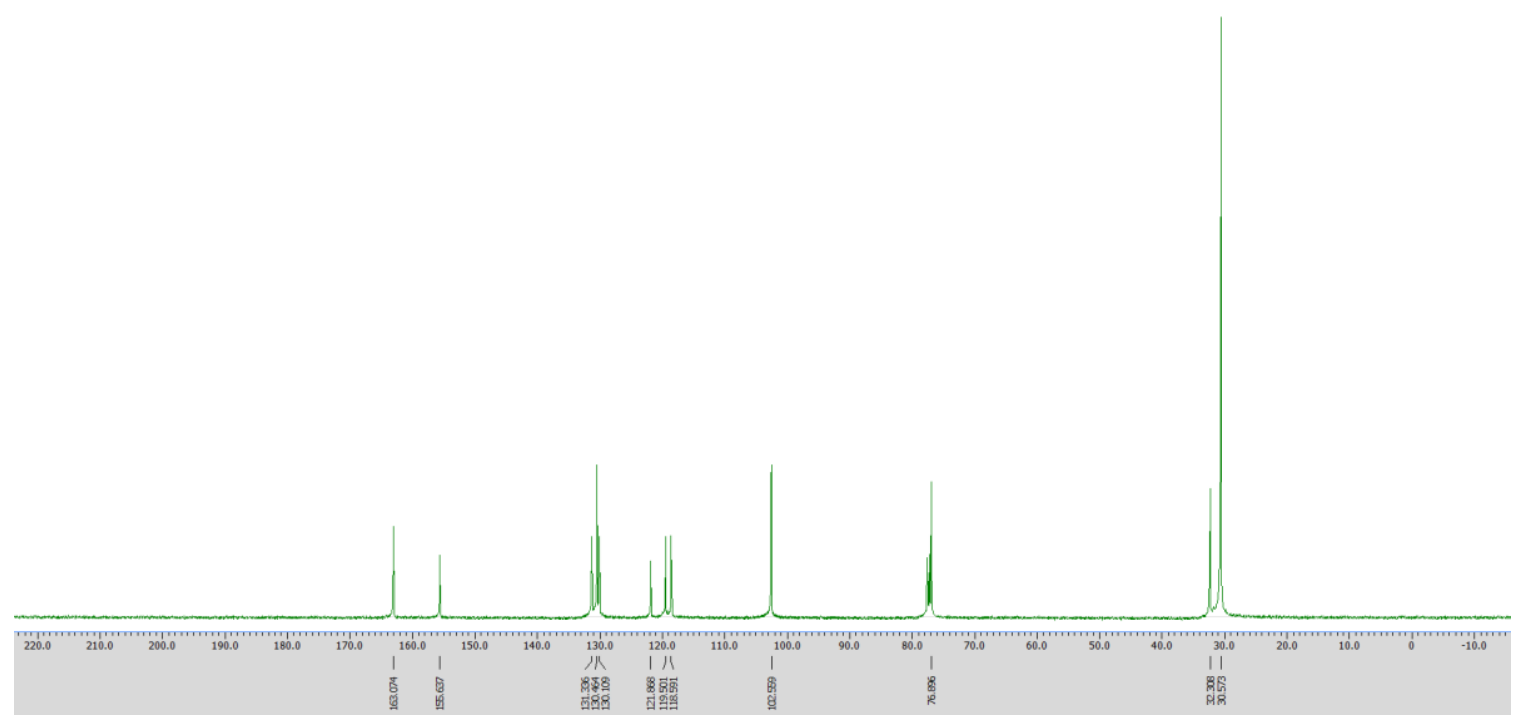

Figure $\mathrm{S} 3.11{ }^{13} \mathrm{C}$ NMR $\left(101 \mathrm{MHz}, \mathrm{CDCl}_{3}\right)$ spectrum of 2-bis(3-tert-butylpyrazol-1yl)methylphenol 
<smiles>O=Cc1ccccc1O</smiles><smiles>O=C(n1ccc(-c2ccccc2)n1)n1ccc(-c2ccccc2)n1</smiles>

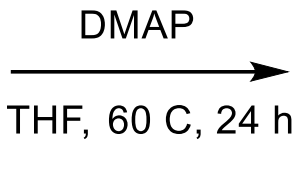<smiles>Oc1ccccc1C(n1ccc(-c2ccccc2)n1)n1ccc(-c2ccccc2)n1</smiles>

2-bis(3-phenylpyrazol-1-yl)methylphenol.

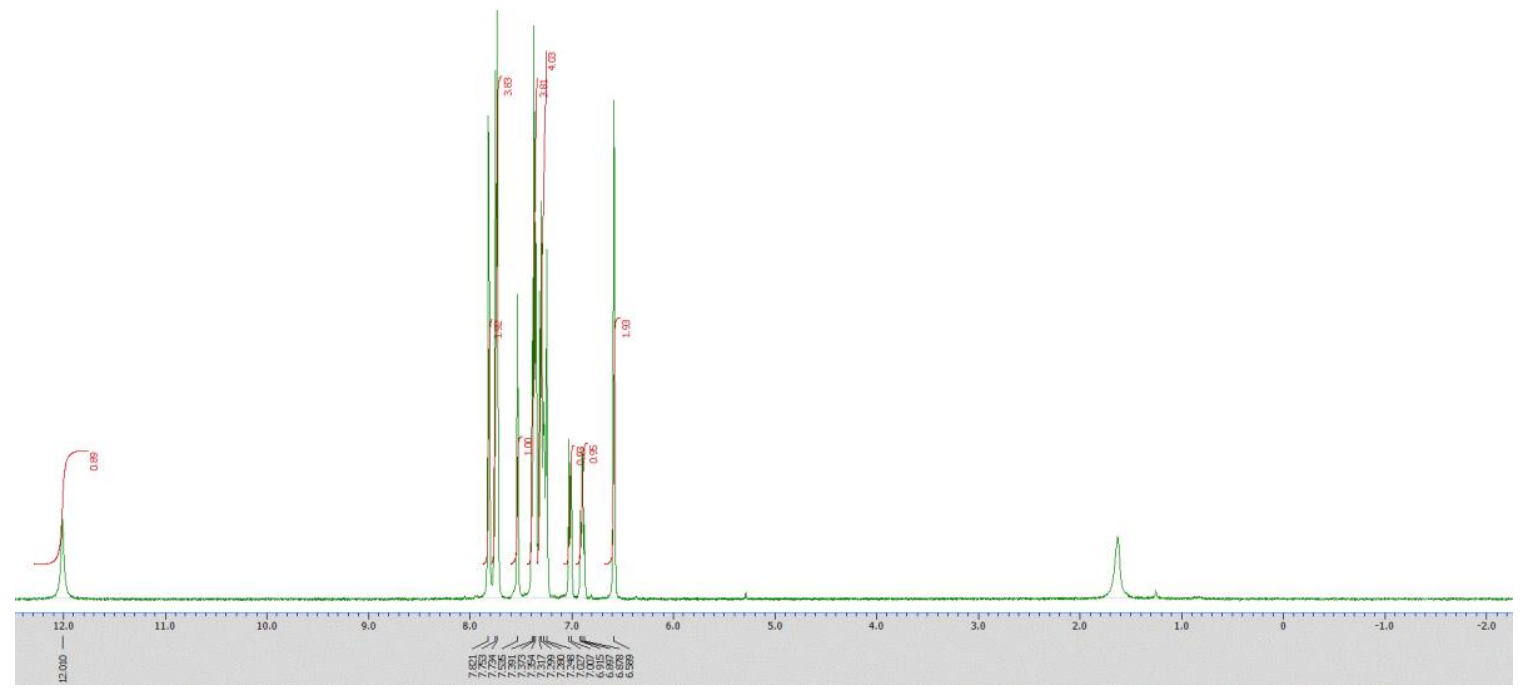

Figure $\mathrm{S} 3.12{ }^{1} \mathrm{H}$ NMR $\left(400 \mathrm{MHz}, \mathrm{CDCl}_{3}\right)$ spectrum of 2-bis(3-phenylpyrazol-1yl)methylphenol.

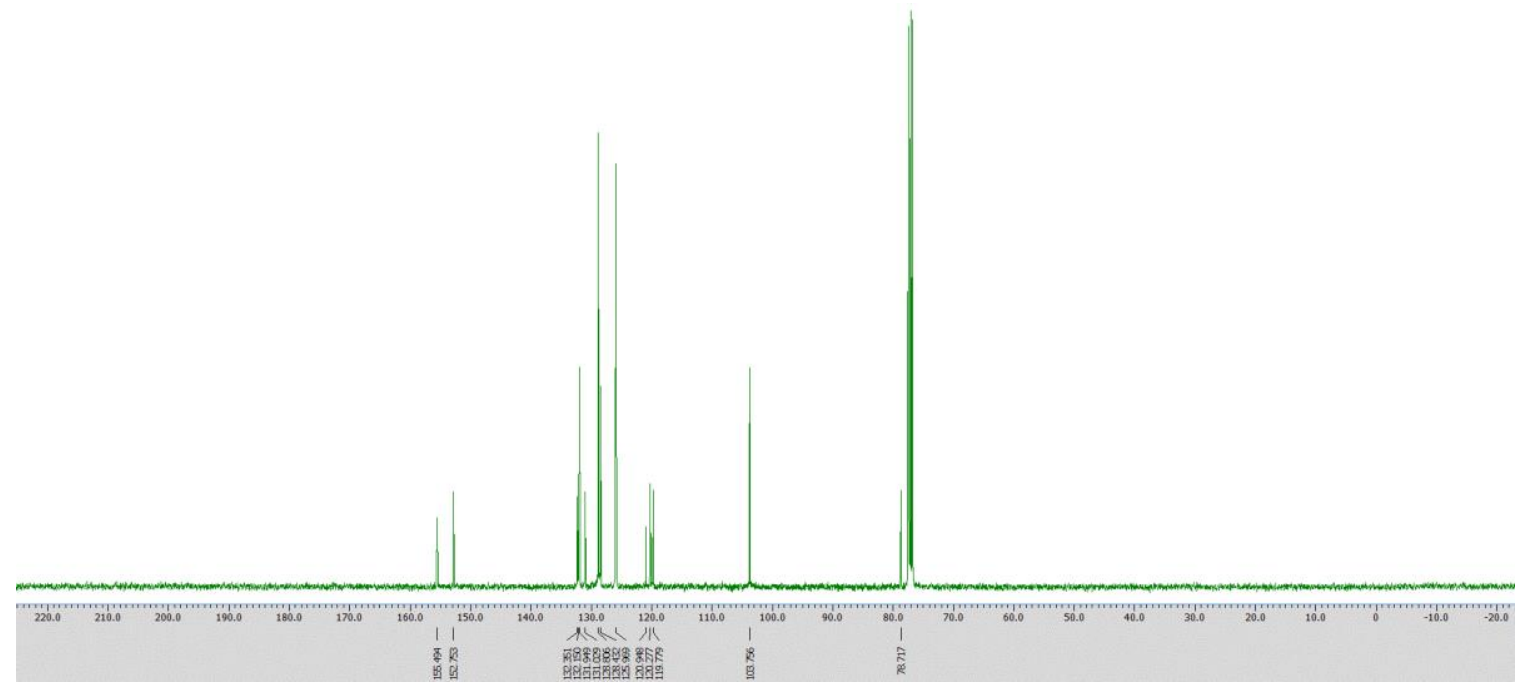


Figure $\mathrm{S} 3.13{ }^{13} \mathrm{C}$ NMR $\left(101 \mathrm{MHz}, \mathrm{CDCl}_{3}\right)$ spectrum of 2-bis(3-phenylpyrazol-1yl)methylphenol<smiles>O=Cc1ccccc1O</smiles><smiles>Cc1ccn(C(=O)n2ccc(C)n2)n1</smiles>
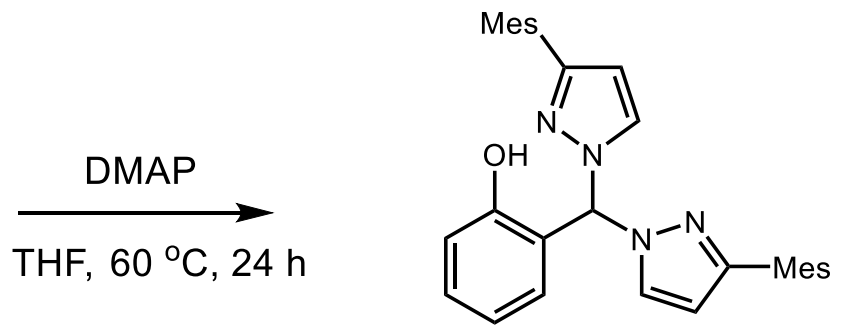

2-bis(3-mesitylpyrazol-1-yl)methyl-phenol.

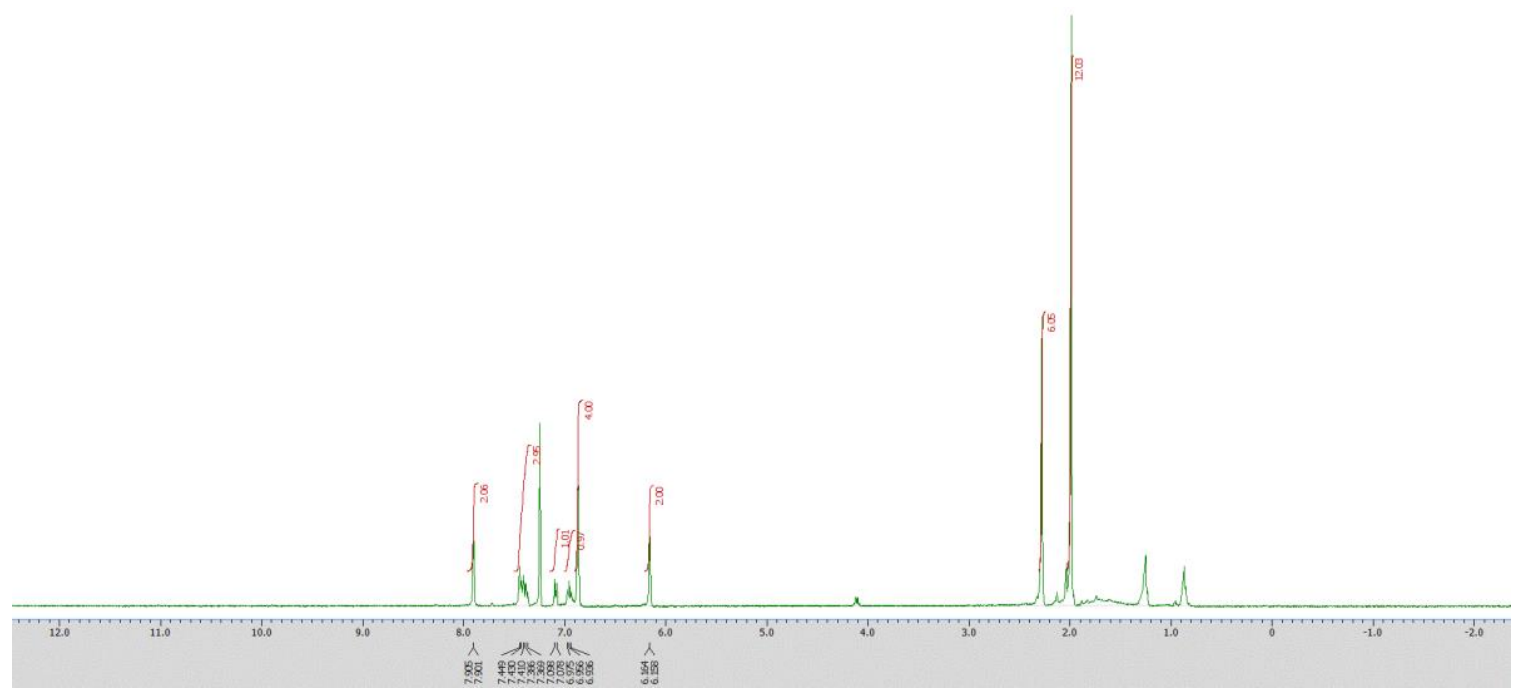

Figure $\mathrm{S} 3.14{ }^{1} \mathrm{H}$ NMR $\left(400 \mathrm{MHz}, \mathrm{CDCl}_{3}\right)$ spectrum of 2-bis(3-mesitylpyrazol-1yl)methyl-phenol. 


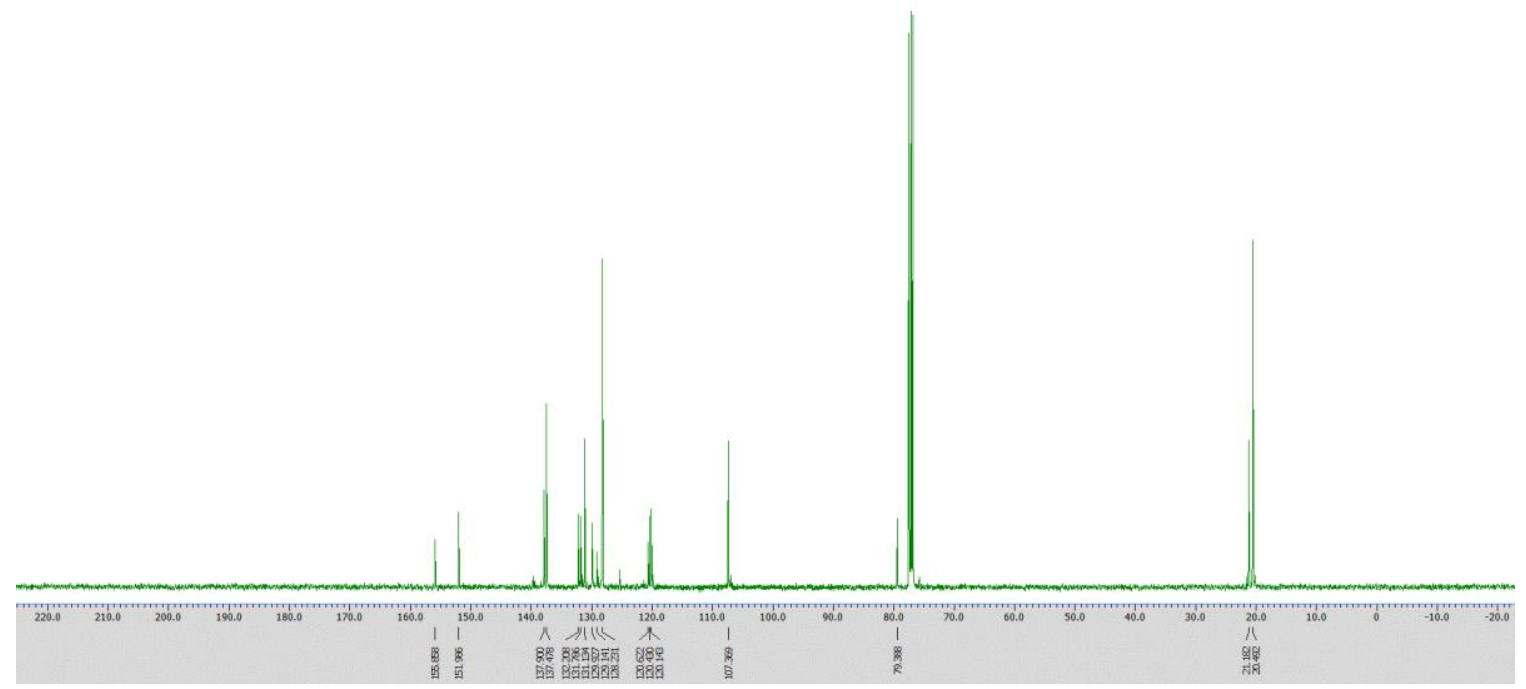

Figure $\mathrm{S} 3.15{ }^{13} \mathrm{C} \mathrm{NMR}\left(101 \mathrm{MHz}, \mathrm{CDCl}_{3}\right)$ spectrum of 2-bis(3-mesitylpyrazol-1-

\section{yl)methyl-phenol}

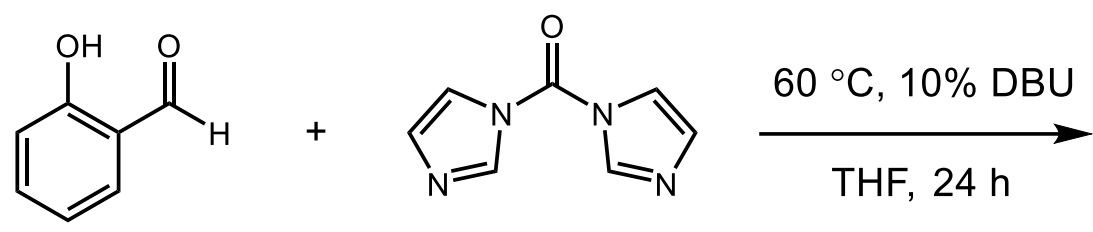<smiles>Oc1ccccc1C(n1ccnc1)n1ccnc1</smiles>

\section{2-bis(imidazol-1-yl)methylphenol.}

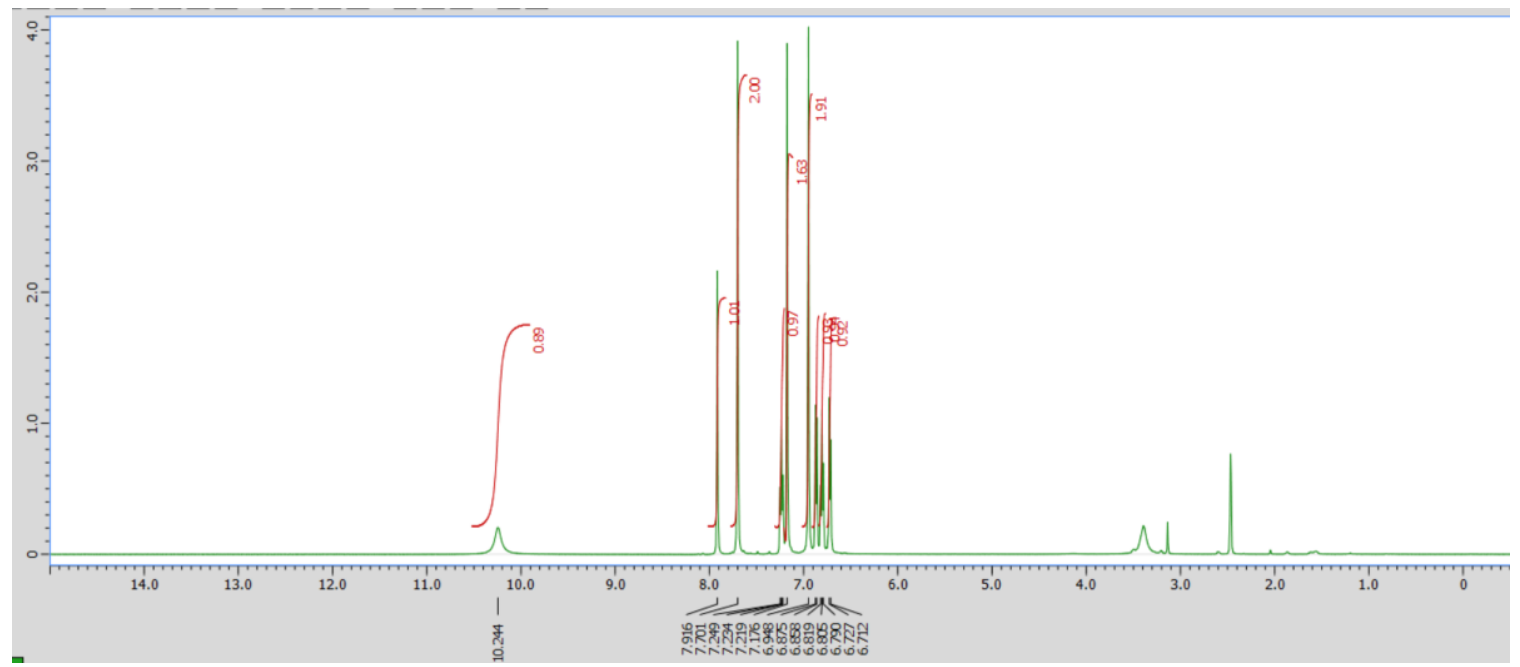


Figure S3.16 ${ }^{1} \mathrm{H}$ NMR (400 $\mathrm{MHz}$, DMSO-d6) spectrum of 2-bis(imidazol-1yl)methylphenol

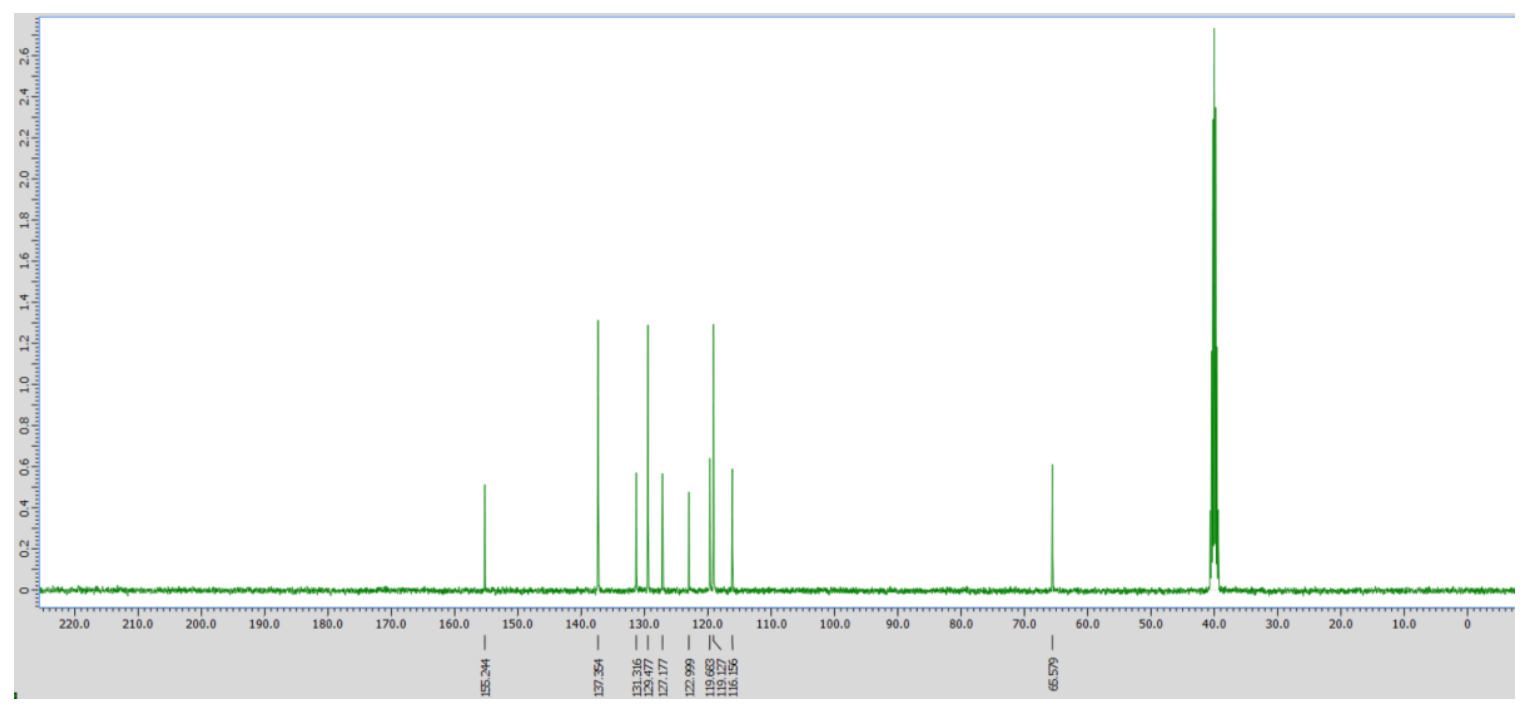

Figure $\mathrm{S} 3.17{ }^{13} \mathrm{C} \quad \mathrm{NMR}(101 \mathrm{MHz}$, DMSO-d6) spectrum of 2-bis(imidazol-1yl)methylphenol<smiles>O=Cc1ccccc1O</smiles><smiles>O=C(n1cncn1)n1cncn1</smiles>

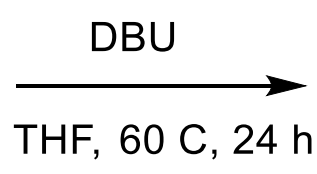<smiles>Oc1ccccc1C(n1cncn1)n1cncn1</smiles>

2-bis(1,2,4-triazol-1-yl)methyl-phenol. 


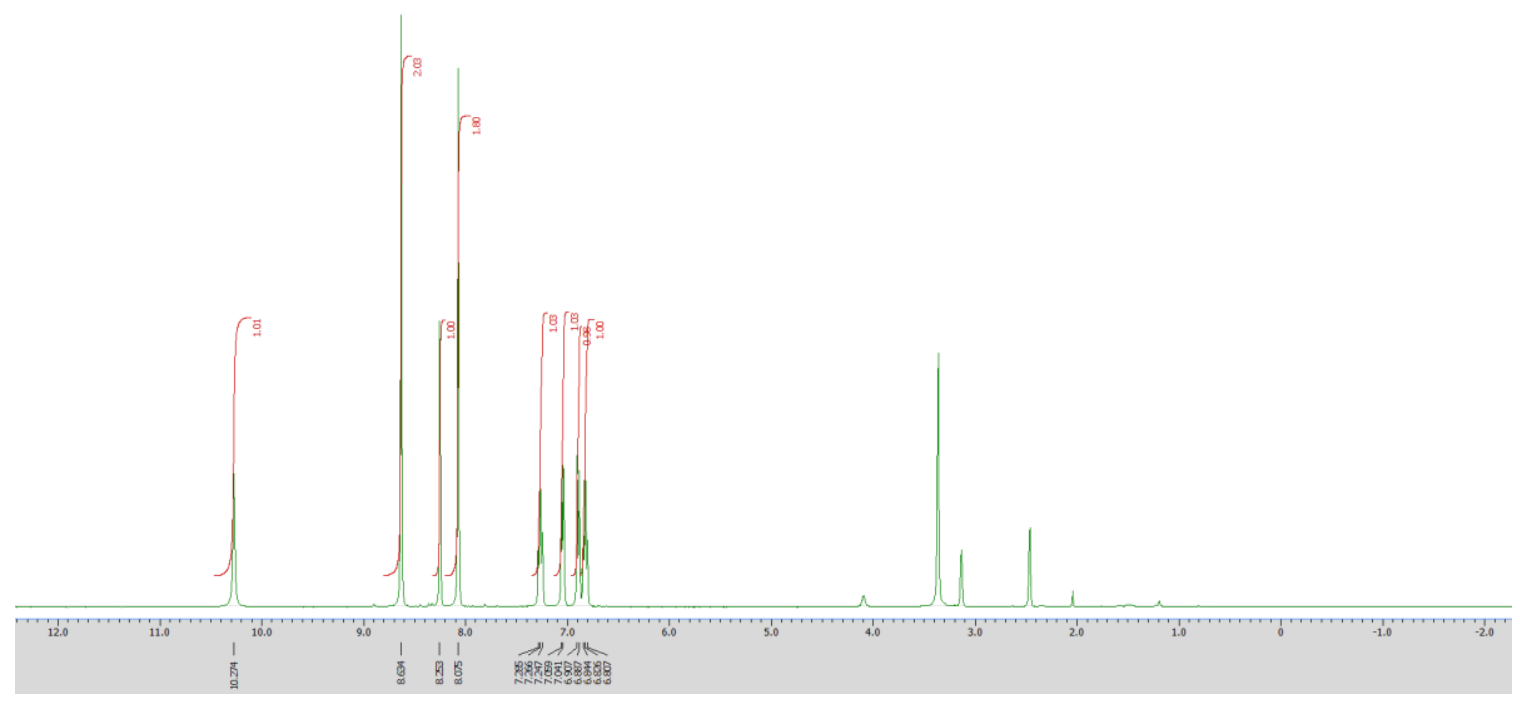

Figure S3.18 ${ }^{1} \mathrm{H}$ NMR (400 MHz, DMSO-d6) spectrum of 2-bis(1,2,4-triazol-1yl)methylphenol

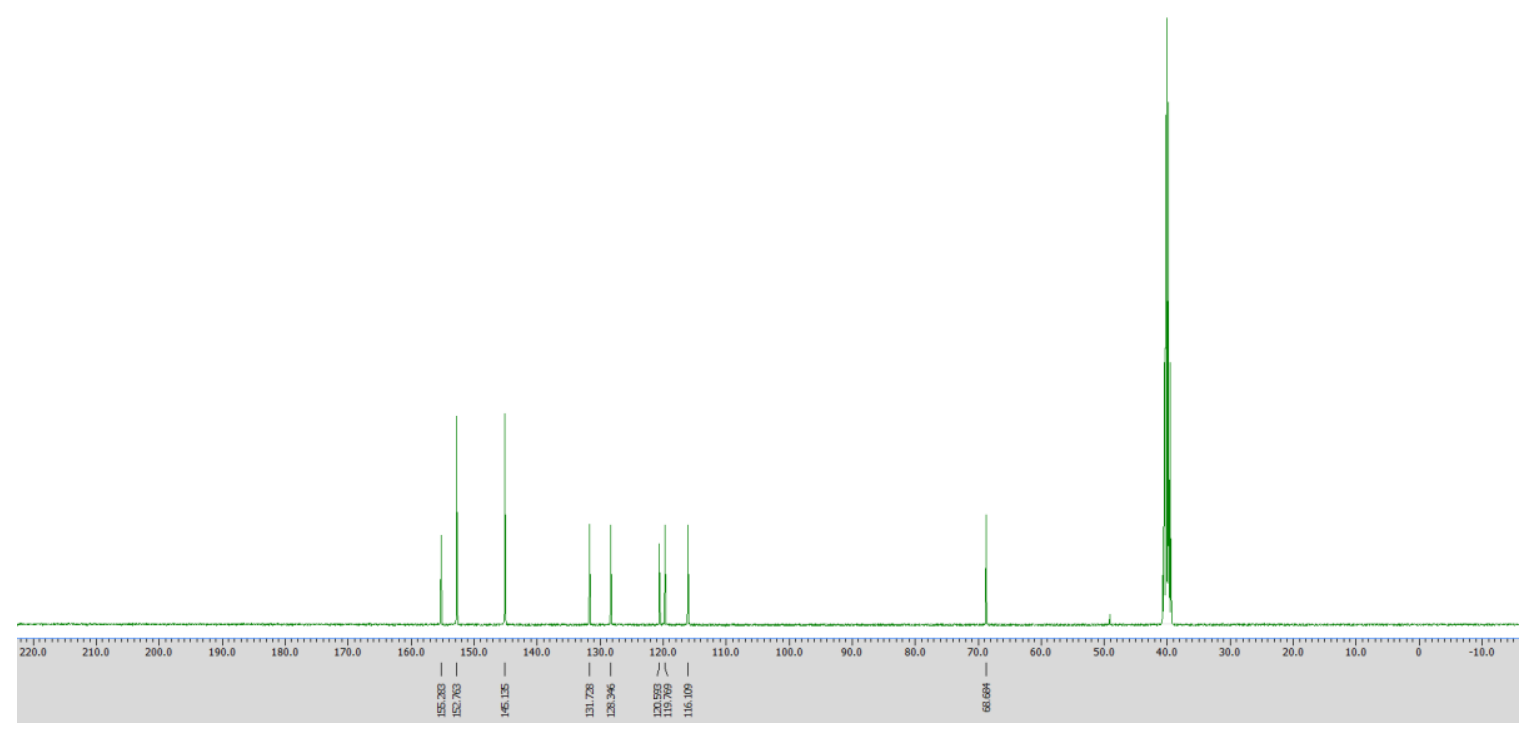

Figure S3.19 ${ }^{13} \mathrm{C}$ NMR (101 MHz, DMSO-d6) spectrum of 2-bis(1,2,4-triazol-1yl)methylphenol 


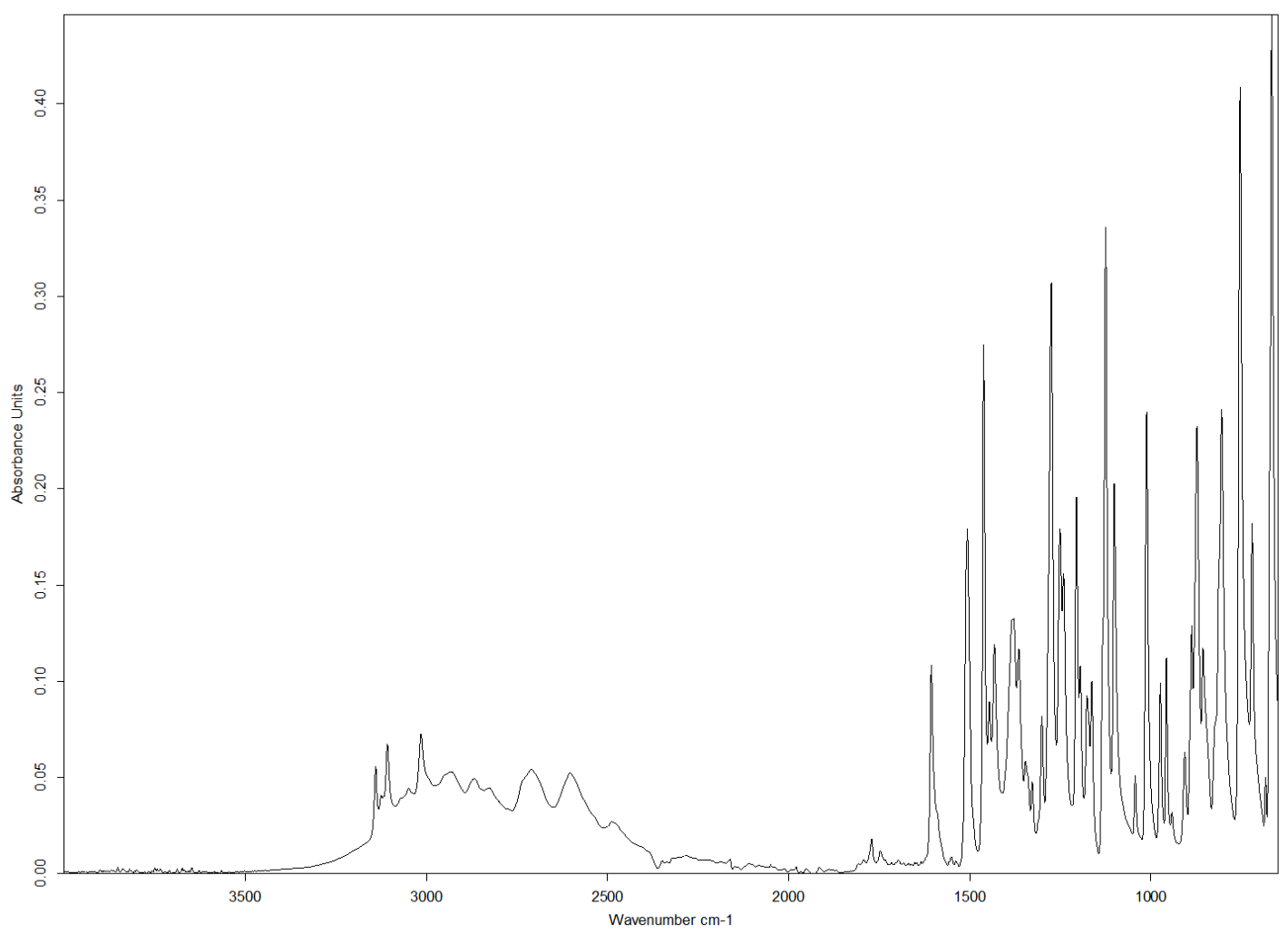

Figure S3.20 IR (Diamond ATR) spectrum of 2-bis(1,2,4-triazol-1-yl)methylphenol

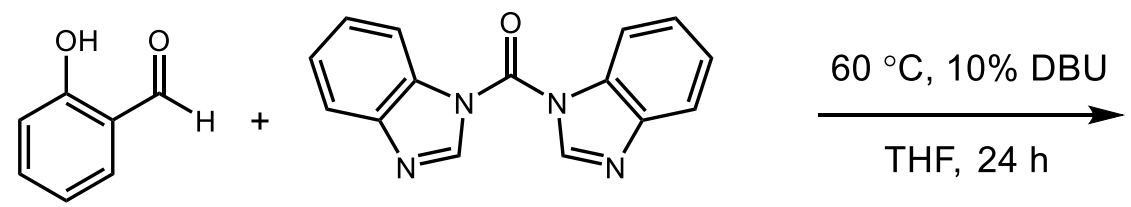<smiles>Oc1ccccc1C(n1cnc2ccccc21)n1cnc2ccccc21</smiles>

\section{2-bis(benzimidazol-1-yl)methylphenol.}




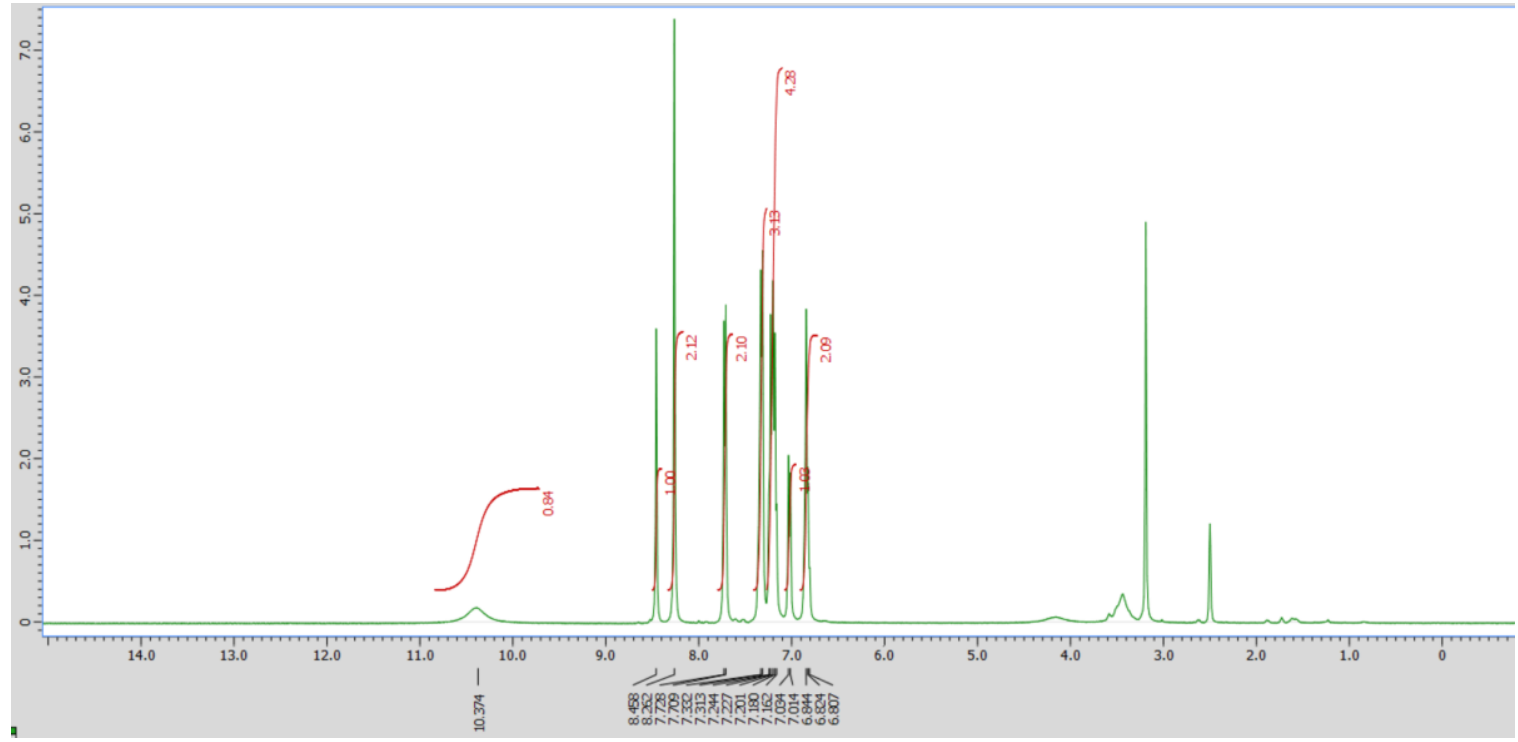

Figure S3.21 ${ }^{1} \mathrm{H}$ NMR (400 MHz, DMSO-d6) spectrum of 2-bis(benzimidazol-1yl)methylphenol

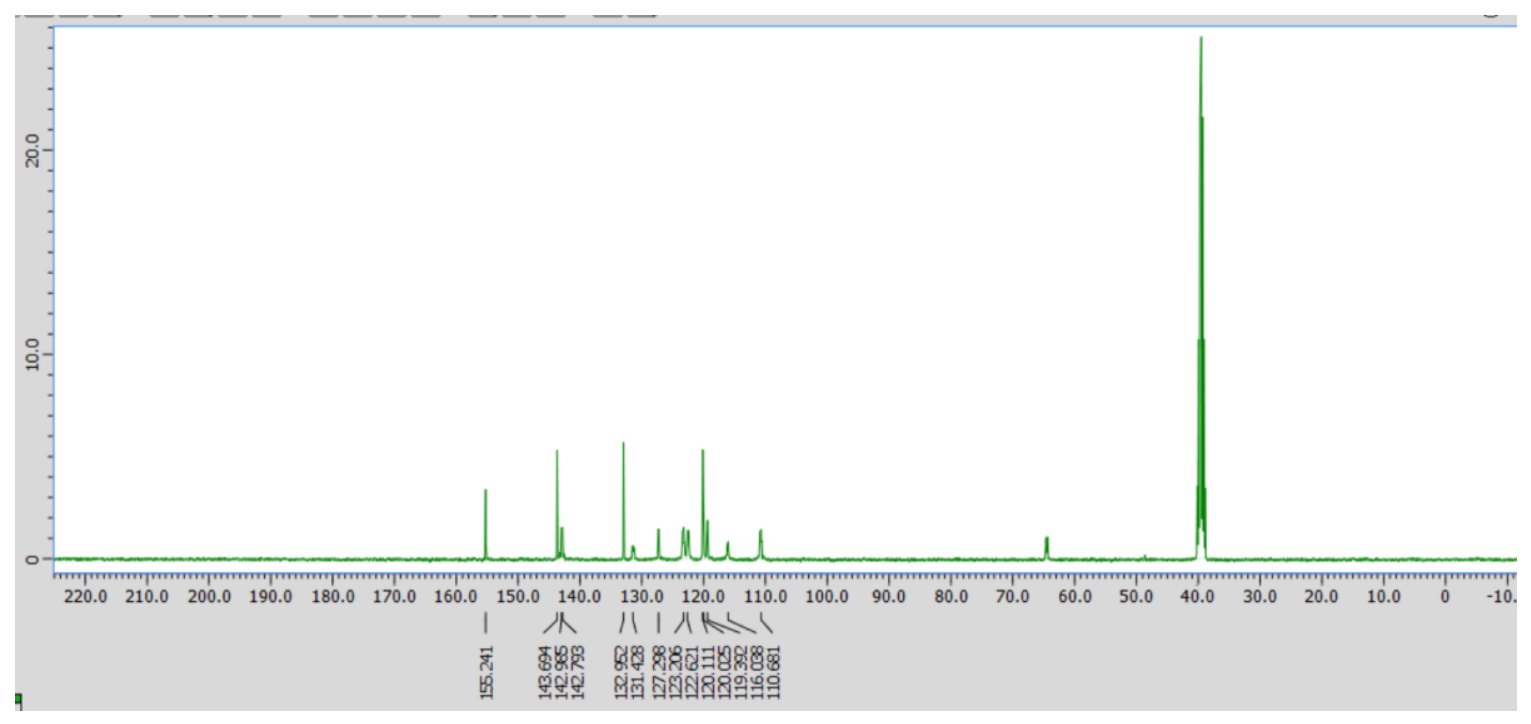

Figure S3.22 ${ }^{13} \mathrm{C}$ NMR (101 MHz, DMSO-d6) spectrum of 2-bis(benzimidazol-1yl)methylphenol 
<smiles>O=Cc1ccccc1O</smiles><smiles>O=C(n1cnc2ccccc21)n1cnc2ccccc21</smiles>

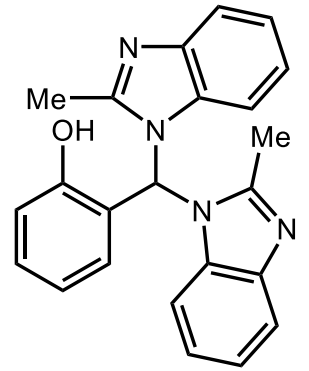

2-bis(2-methyl-benzimidazol-1-yl)methylphenol.

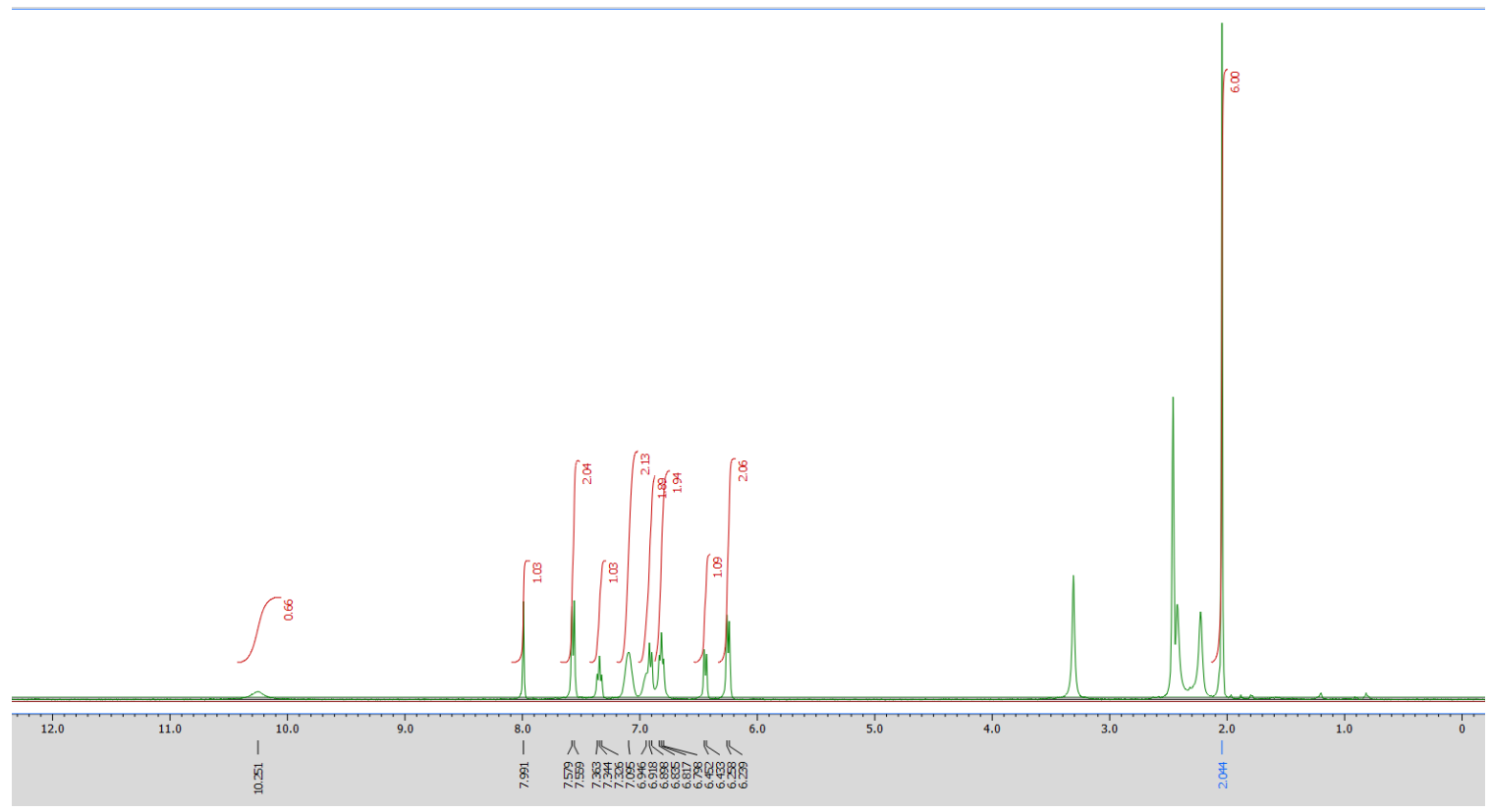

Figure S3.23 ${ }^{1} \mathrm{H}$ NMR (400 MHz, DMSO-d6) spectrum of 2-bis(2-methylbenzimidazol1-yl)methylphenol 


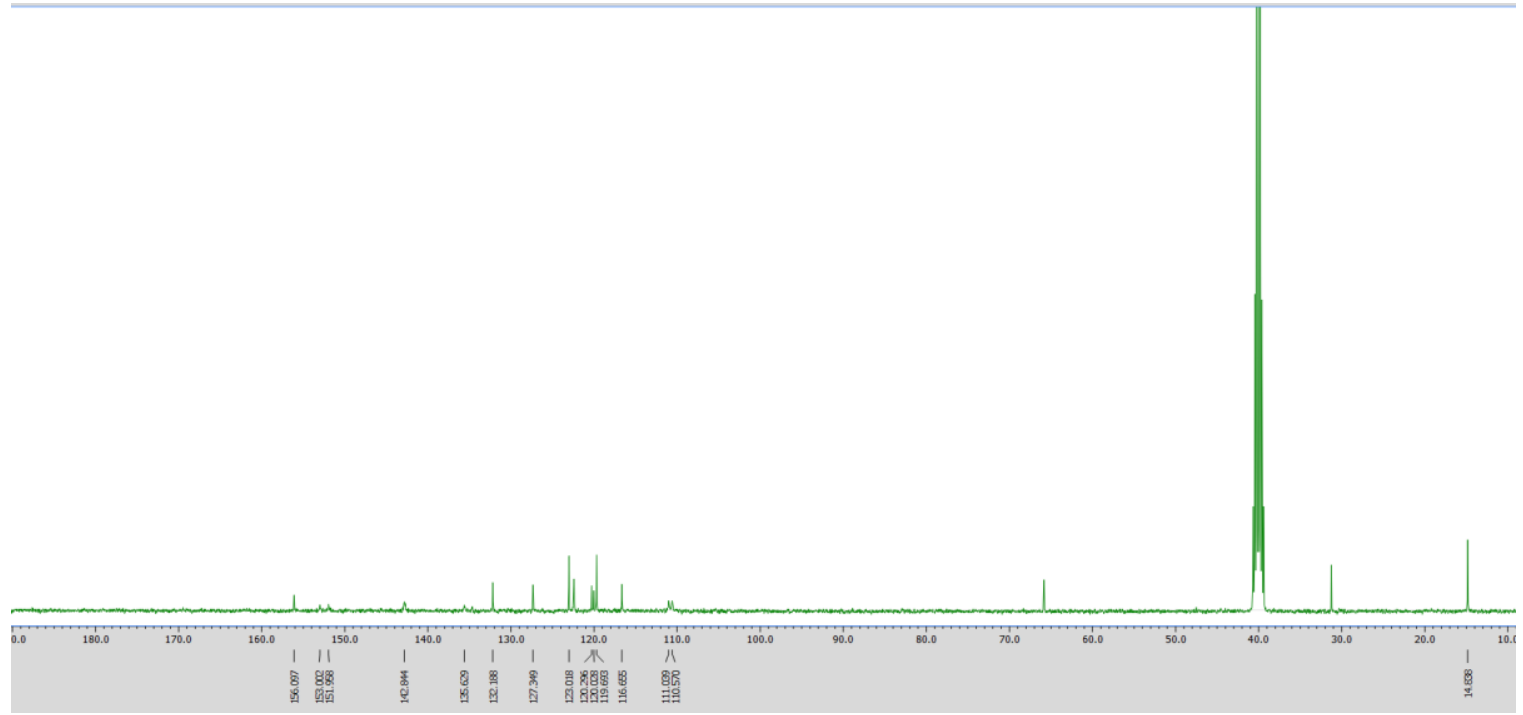

Figure S3.24 ${ }^{13} \mathrm{C}$ NMR (100 MHz, DMSO-d6) spectrum of 2-bis(2-methylbenzimidazol1-yl)methylphenol

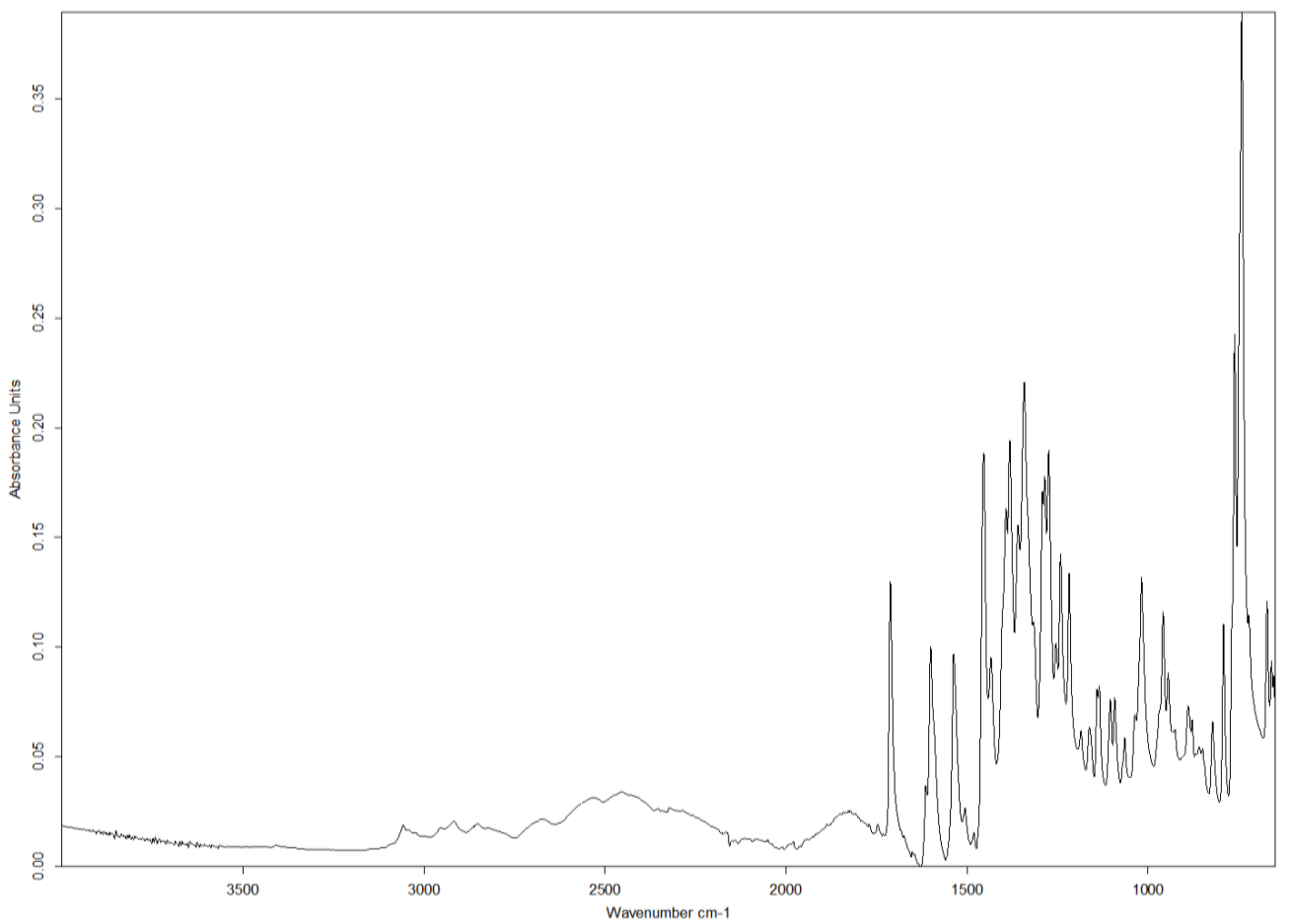

Figure S3.25 IR (Diamond ATR) spectrum of 2-bis(2-methylbenzimidazol-1yl)methylphenol 
<smiles>O=Cc1ccccc1</smiles><smiles>Cc1cc(C)n(C(=O)n2nc(C)cc2C)n1</smiles><smiles>Cc1cc(C)n(C(c2ccccc2)n2nc(C)cc2C)n1</smiles>

\section{bis(3,5-dimethylpyrazol-1-yl)methylbenzene.}

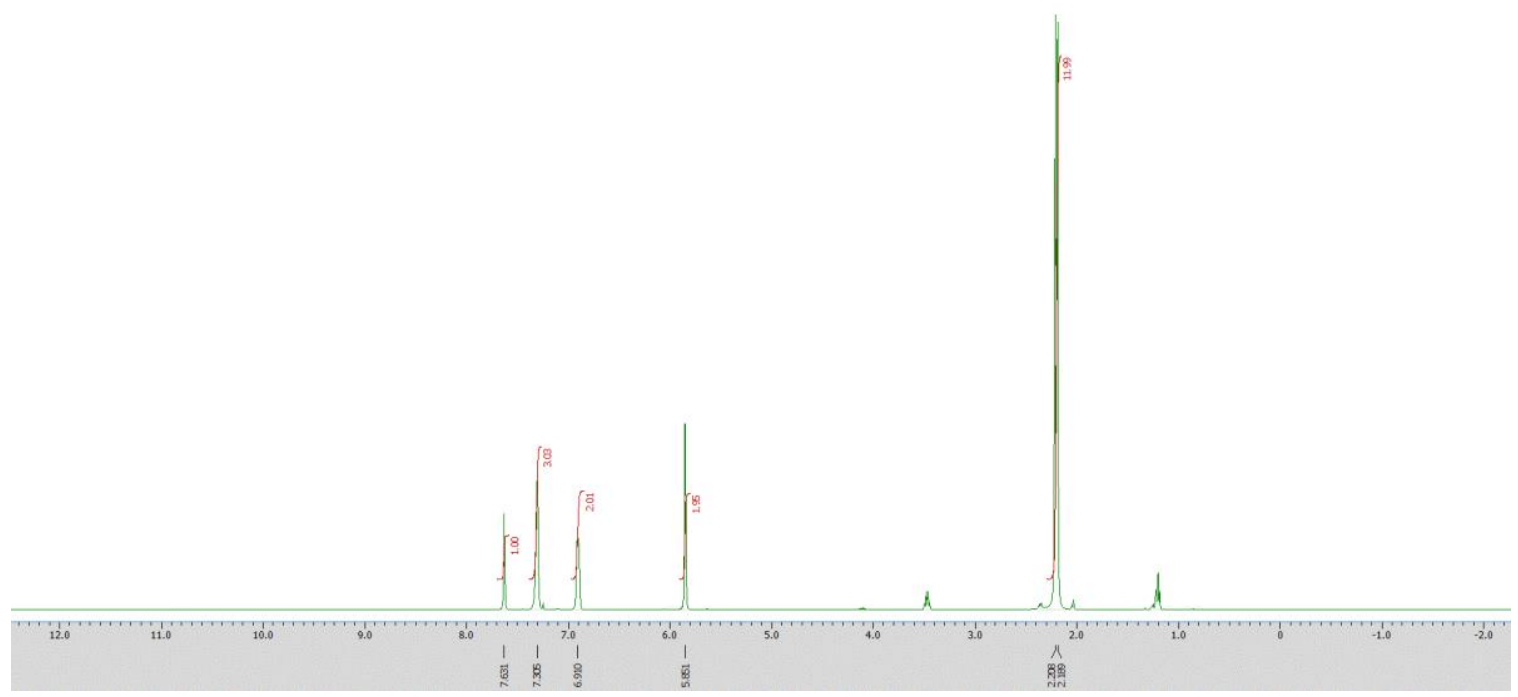

Figure S3.26 ${ }^{1} \mathrm{H}$ NMR (400 $\left.\mathrm{MHz}, \mathrm{CDCl}_{3}\right)$ spectrum of bis(3,5-dimethylpyrazol-1yl)methylbenzene

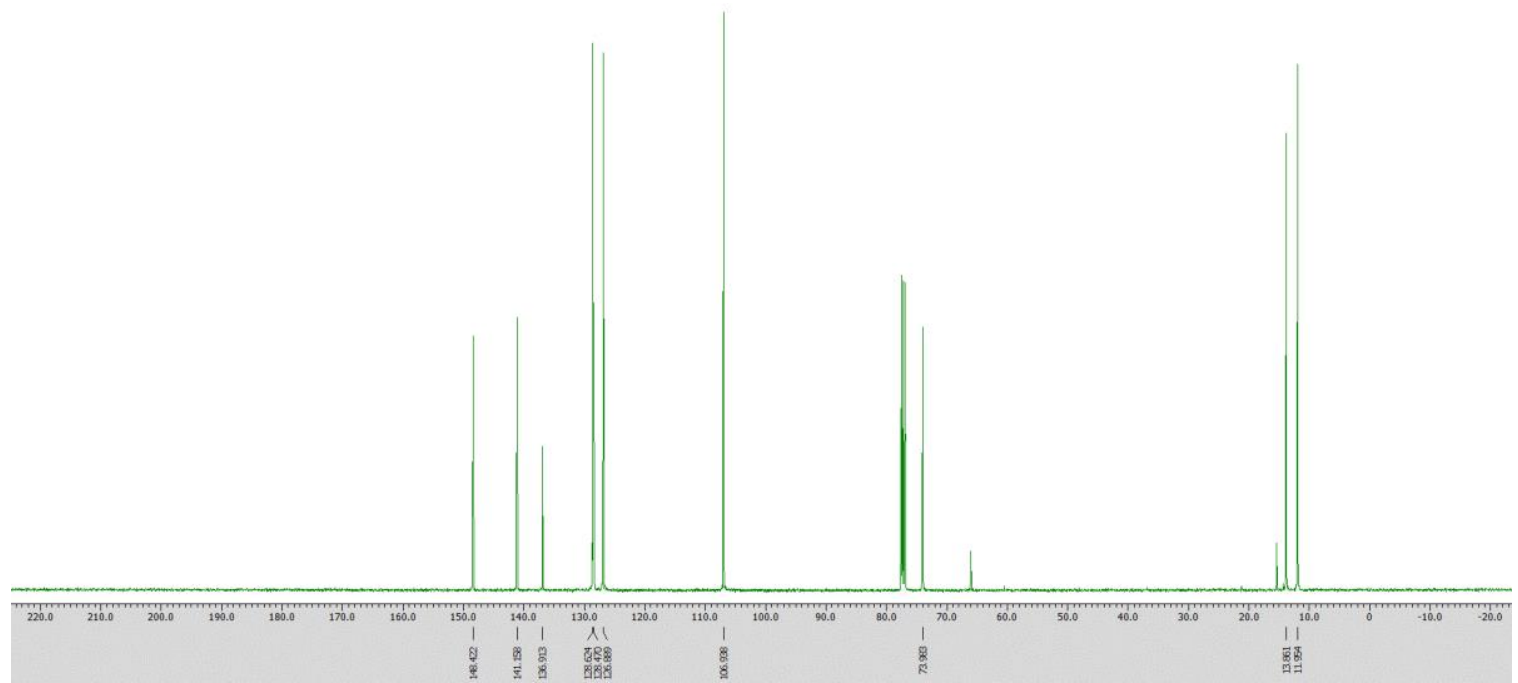


Figure S3.27 ${ }^{13} \mathrm{C}$ NMR $\left(101 \mathrm{MHz}, \mathrm{CDCl}_{3}\right)$ spectrum of bis(3,5-dimethylpyrazol-1yl)methylbenzene<smiles>COc1ccc(C=O)cc1</smiles><smiles>Cc1cc(C)n(C(=O)n2nc(C)cc2C)n1</smiles><smiles>COc1ccc(C(n2nc(C)cc2C)n2nc(C)cc2C)cc1</smiles>

1-bis(3,5-dimethylpyrazol-1-yl)methyl-4-methoxybenzene.

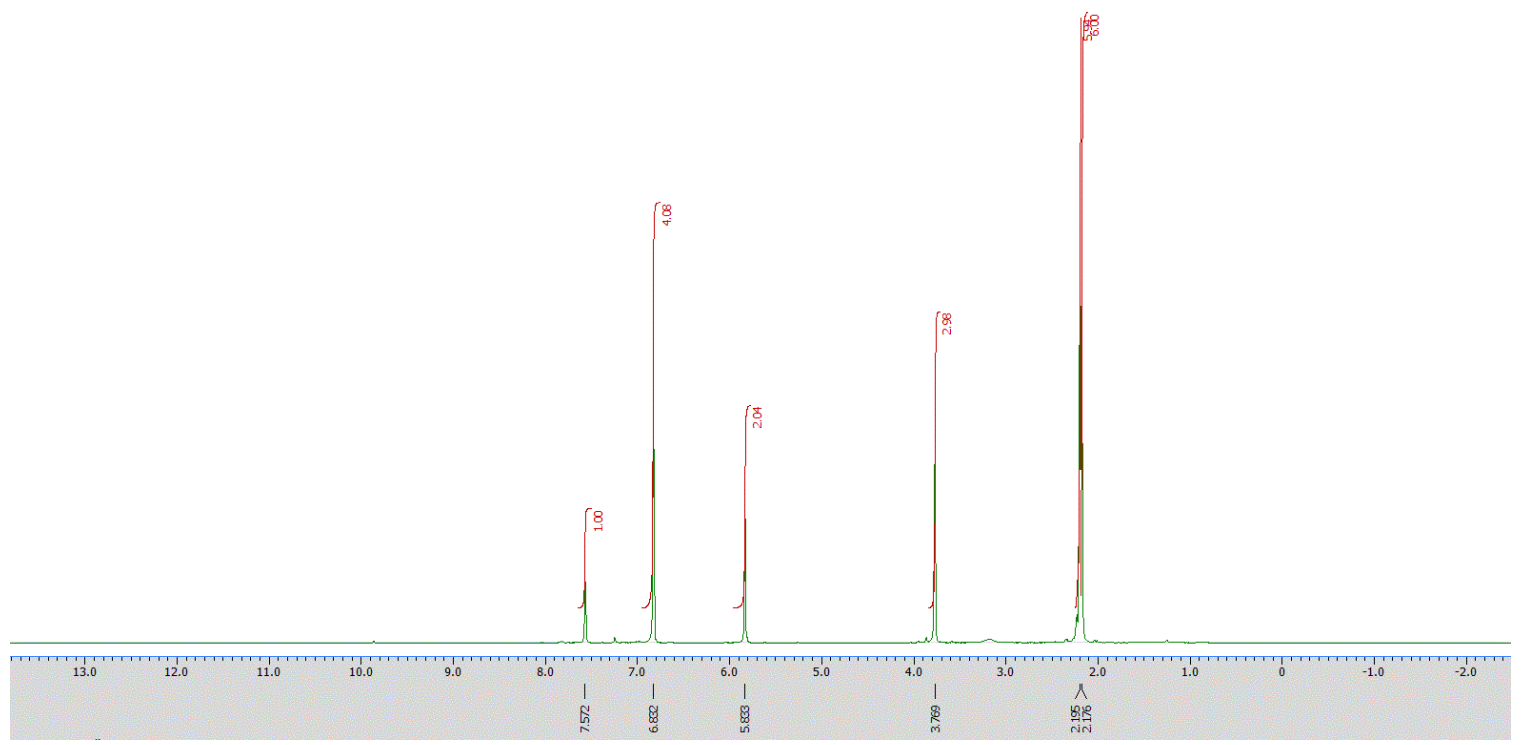

Figure S3.28 ${ }^{1} \mathrm{H}$ NMR (400 MHz, $\left.\mathrm{CDCl}_{3}\right)$ spectrum of 1-bis(3,5-dimethylpyrazol-1yl)methyl-4-methoxybenzene 


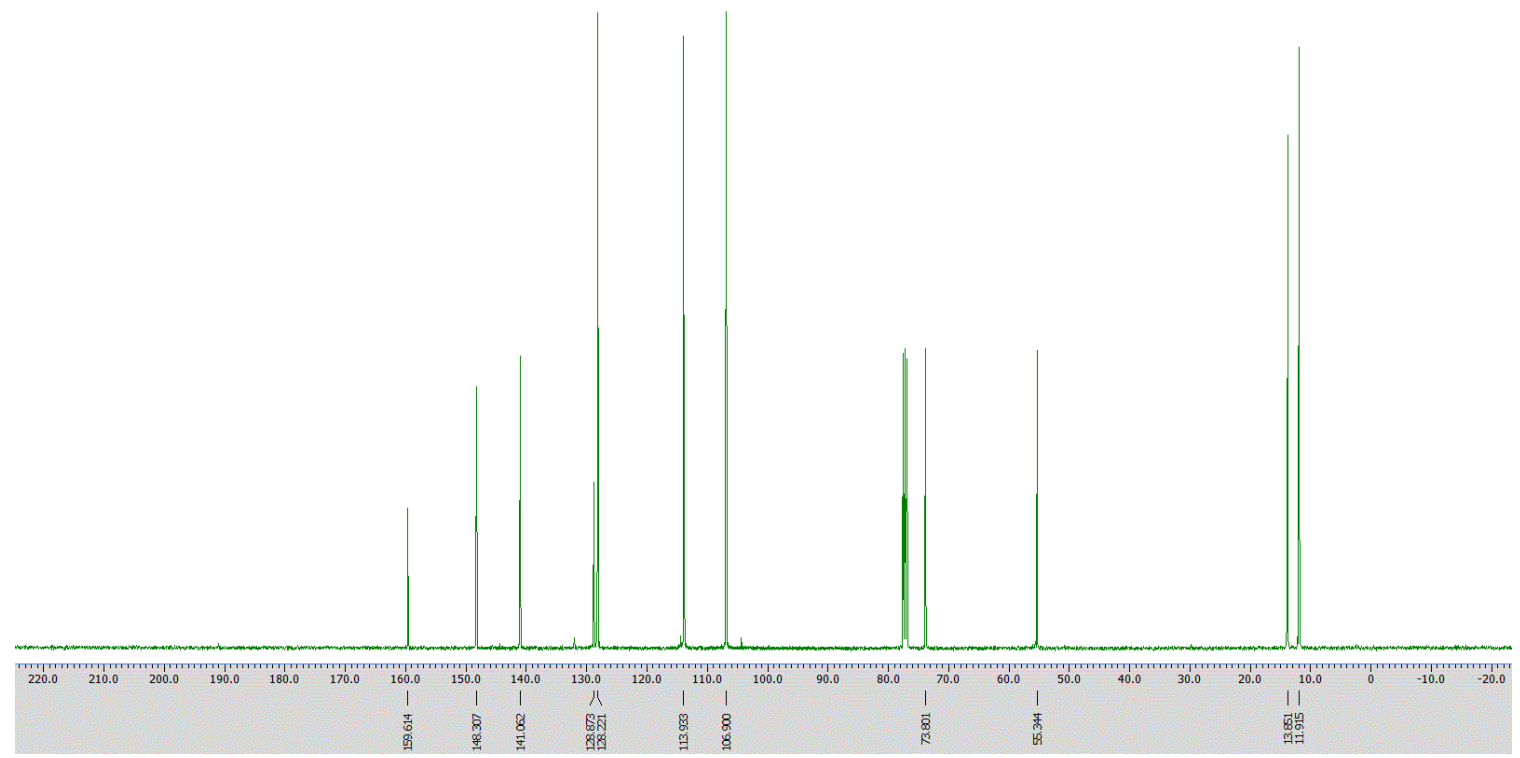

Figure S3.29 ${ }^{13} \mathrm{C}$ NMR (101 $\left.\mathrm{MHz}, \mathrm{CDCl}_{3}\right)$ spectrum of 1-bis(3,5-dimethylpyrazol-1yl)methyl-4-methoxybenzene

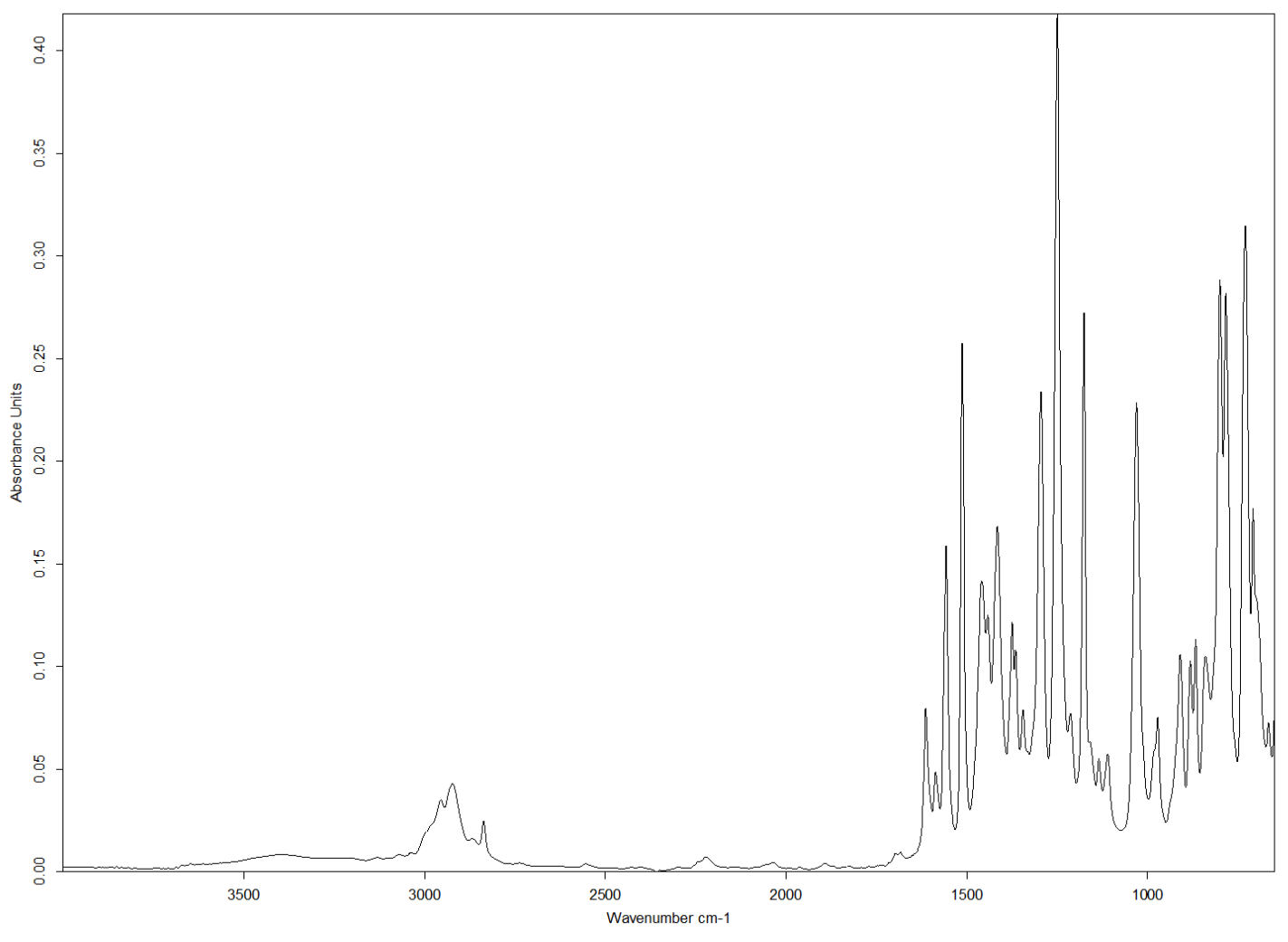


Figure S3.30 IR (Diamond ATR) spectrum of 1-bis(3,5-dimethylpyrazol-1-yl)methyl-4methoxybenzene<smiles>Cc1ccc(C=O)cc1</smiles><smiles>Cc1cc(C)n(C(=O)n2nc([N+](=O)[O-])cc2C)n1</smiles>

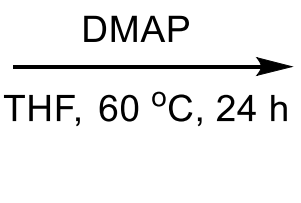<smiles>Cc1ccc(C(n2nc(C)cc2C)n2nc(C)cc2C)cc1</smiles>

1-bis(3,5-dimethylpyrazol-1-yl)methyl-4-methylbenzene.

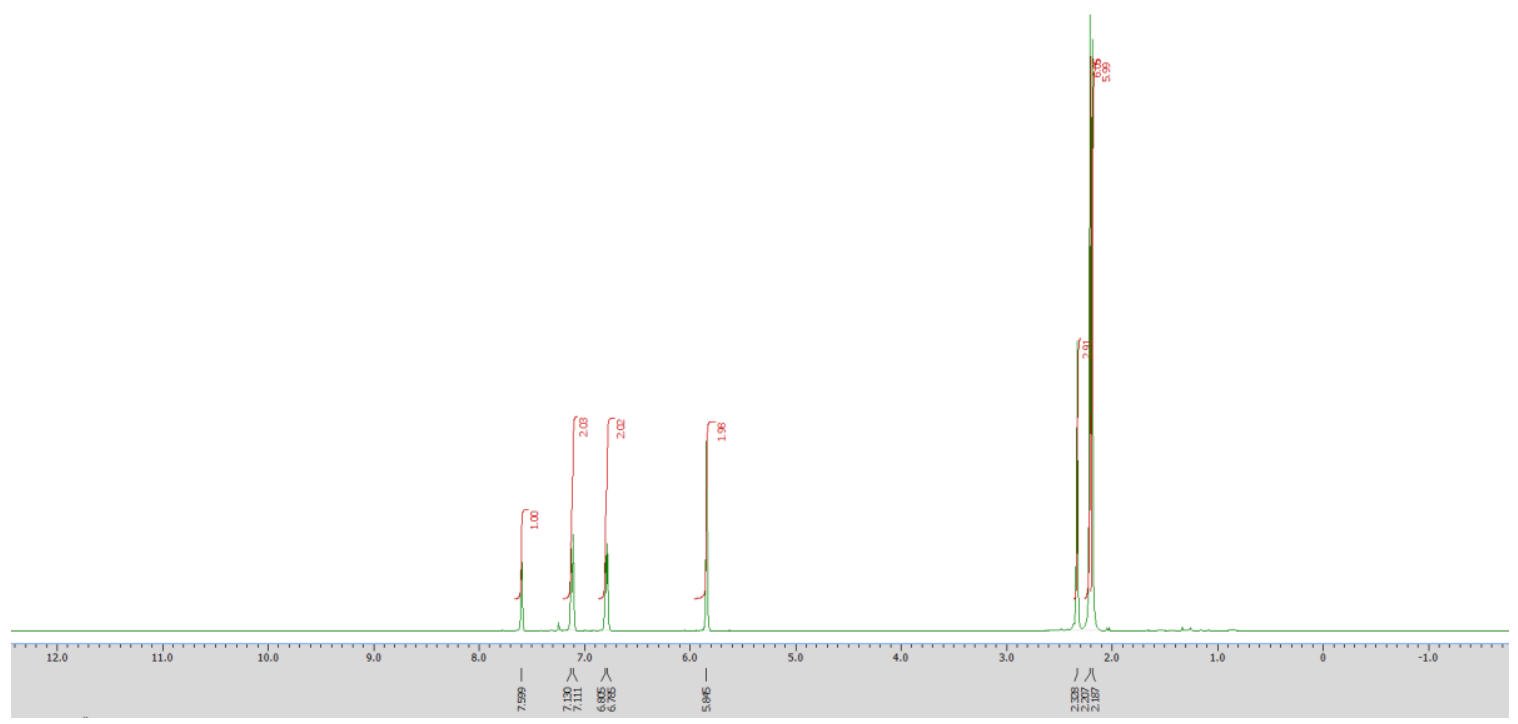


Figure $\mathrm{S} 3.31{ }^{1} \mathrm{H}$ NMR $\left(400 \mathrm{MHz}, \mathrm{CDCl}_{3}\right)$ spectrum of 1-bis(3,5-dimethylpyrazol-1yl)methyl-4-methylbenzene

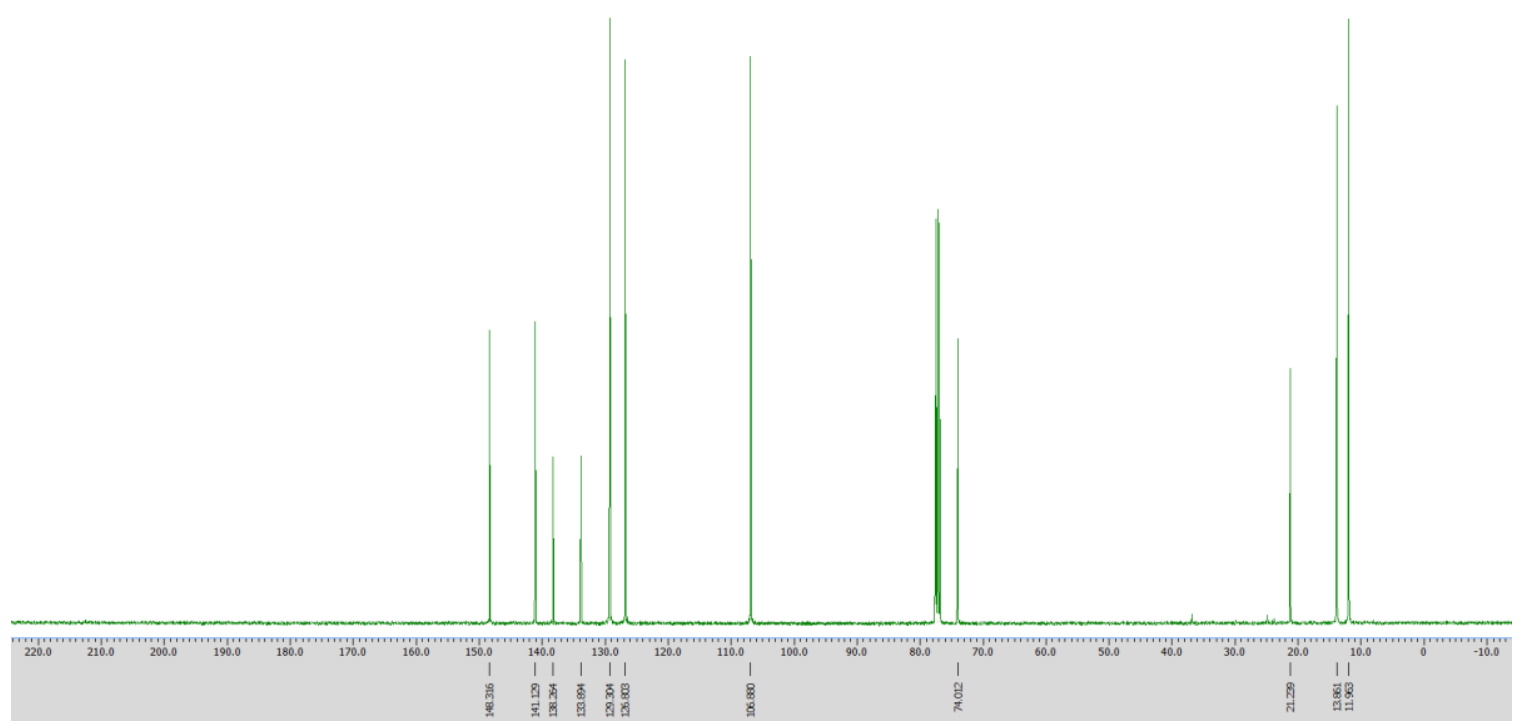

Figure $\mathrm{S} 3.32{ }^{13} \mathrm{C}$ NMR (101 MHz, $\mathrm{CDCl}_{3}$ ) spectrum of 1-bis(3,5-dimethylpyrazol-1yl)methyl-4-methylbenzene 


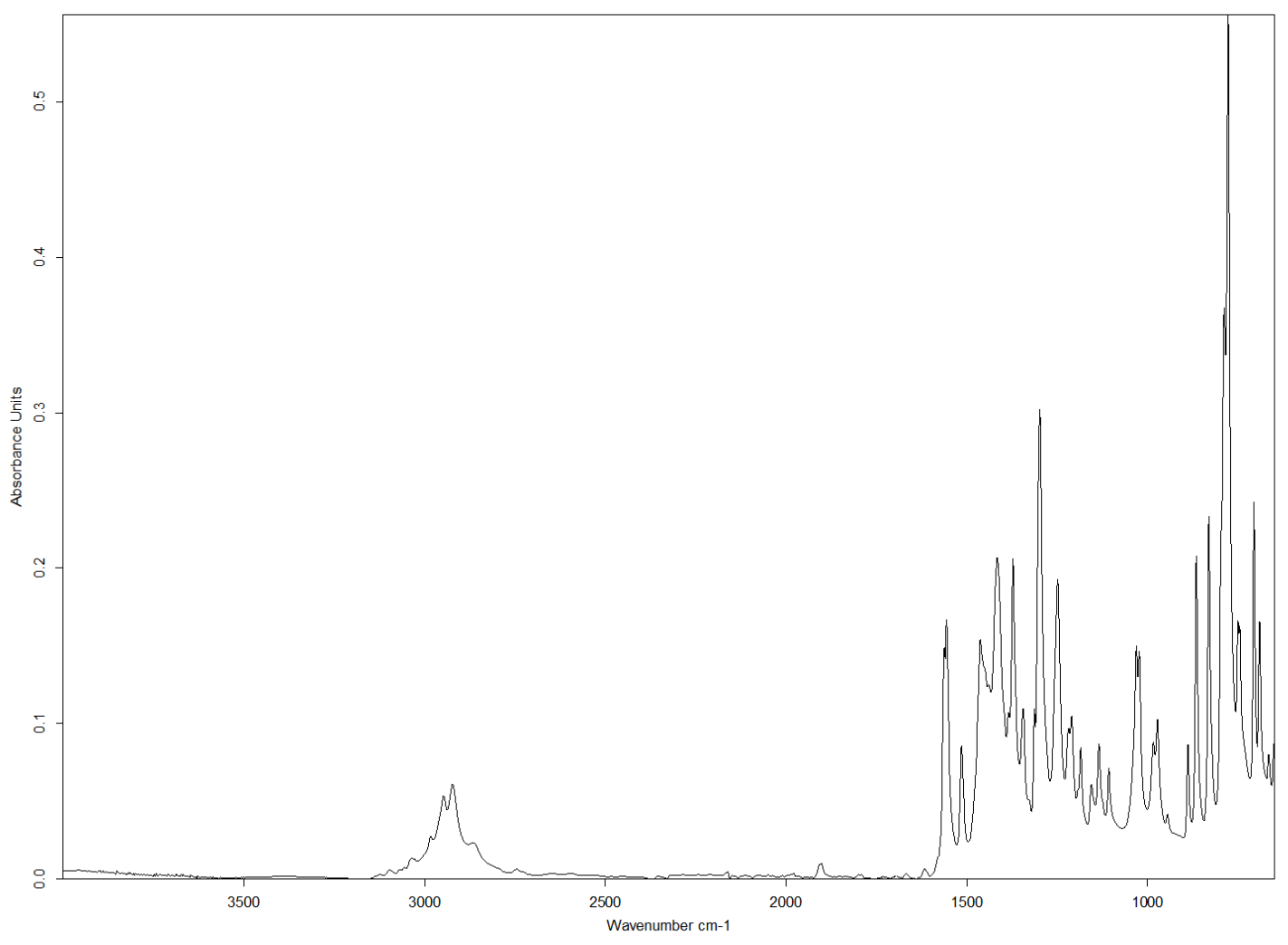

Figure S3.33 IR (Diamond ATR) spectrum of 1-bis(3,5-dimethylpyrazol-1-yl)methyl-4methylbenzene

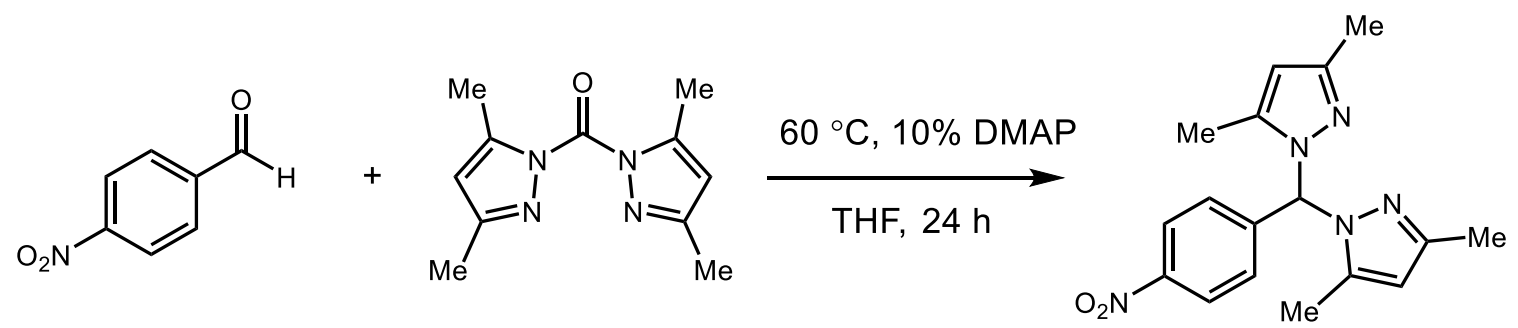

4-bis(3,5-dimethylpyrazol-1-yl)methyl-nitrobenzene. 


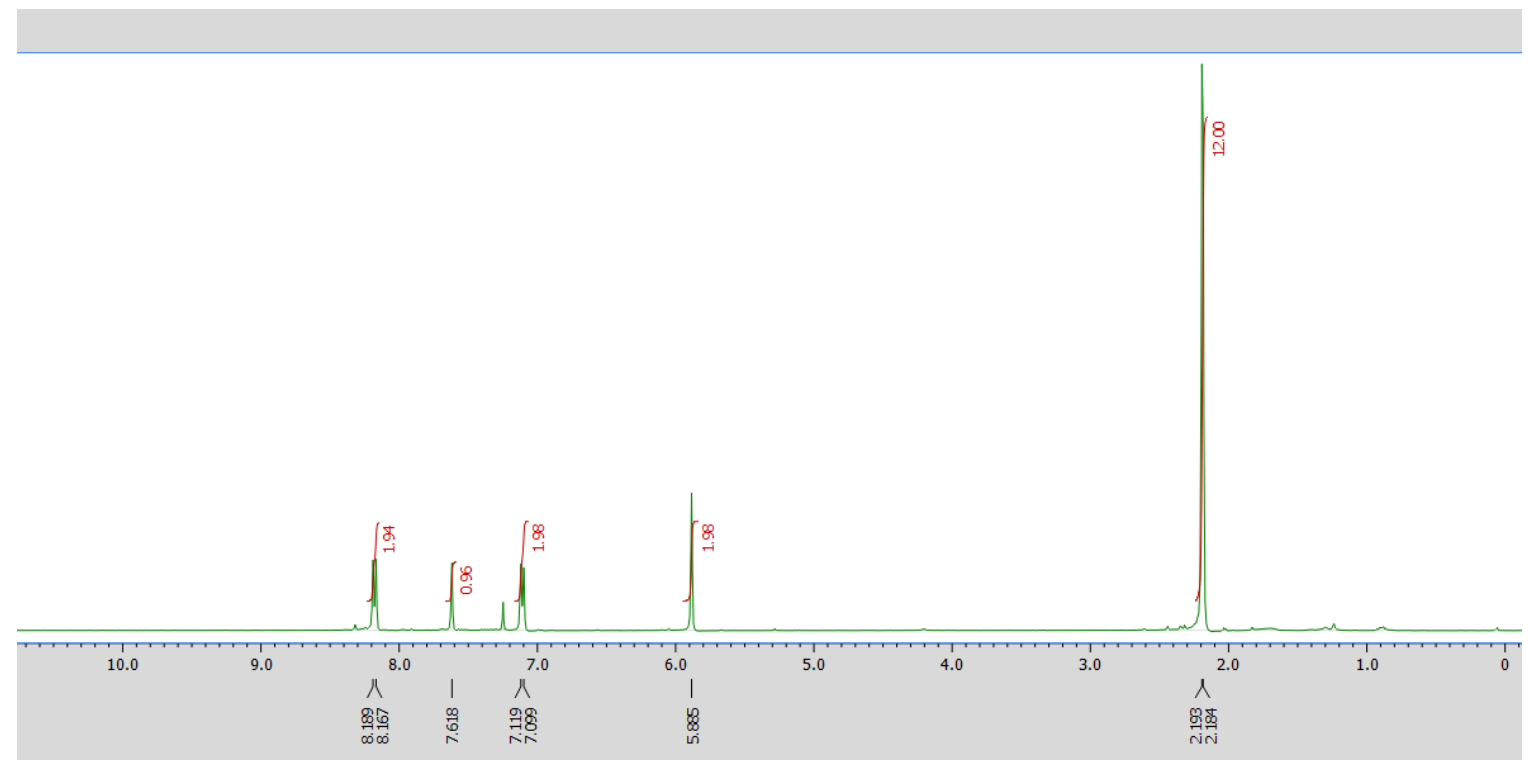

Figure $\mathrm{S} 3.34{ }^{1} \mathrm{H}$ NMR (400 MHz, $\mathrm{CDCl}_{3}$ ) spectrum of 4-bis(3,5-dimethylpyrazol-1yl)methyl-nitrobenzene.

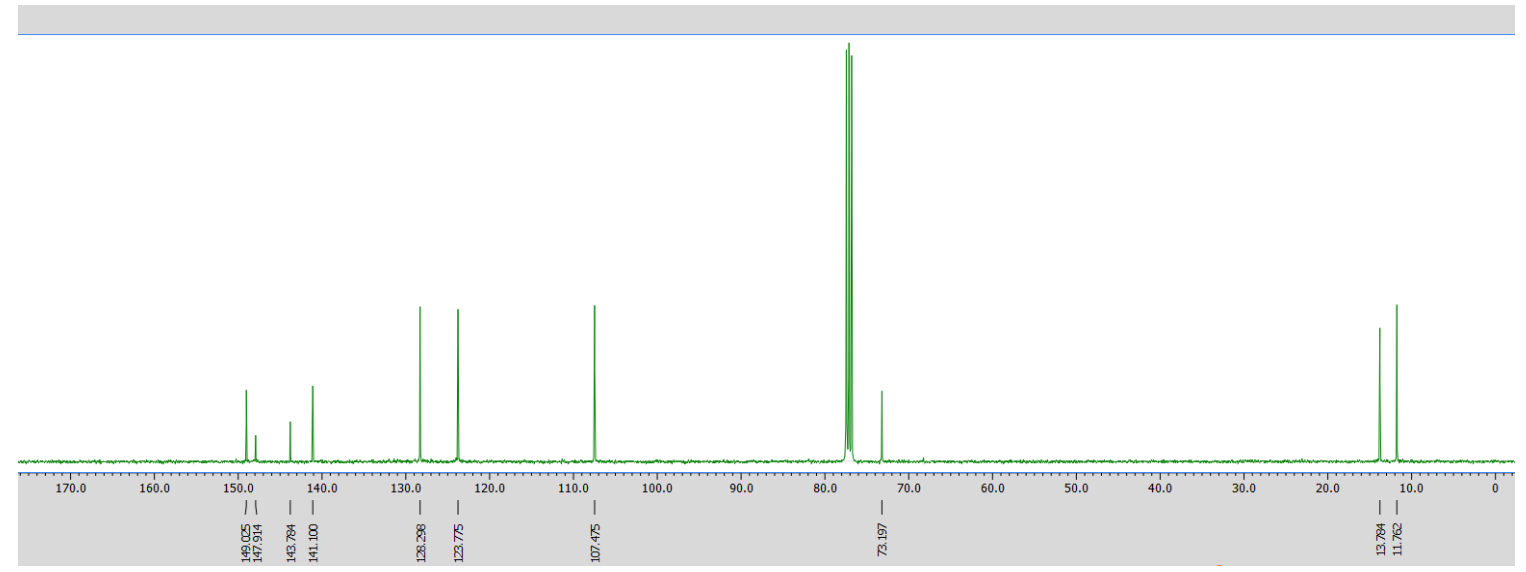

Figure $\mathrm{S} 3.35{ }^{13} \mathrm{C}$ NMR (100 MHz, $\left.\mathrm{CDCl}_{3}\right)$ spectrum of 4-bis(3,5-dimethylpyrazol-1yl)methyl-nitrobenzene. 


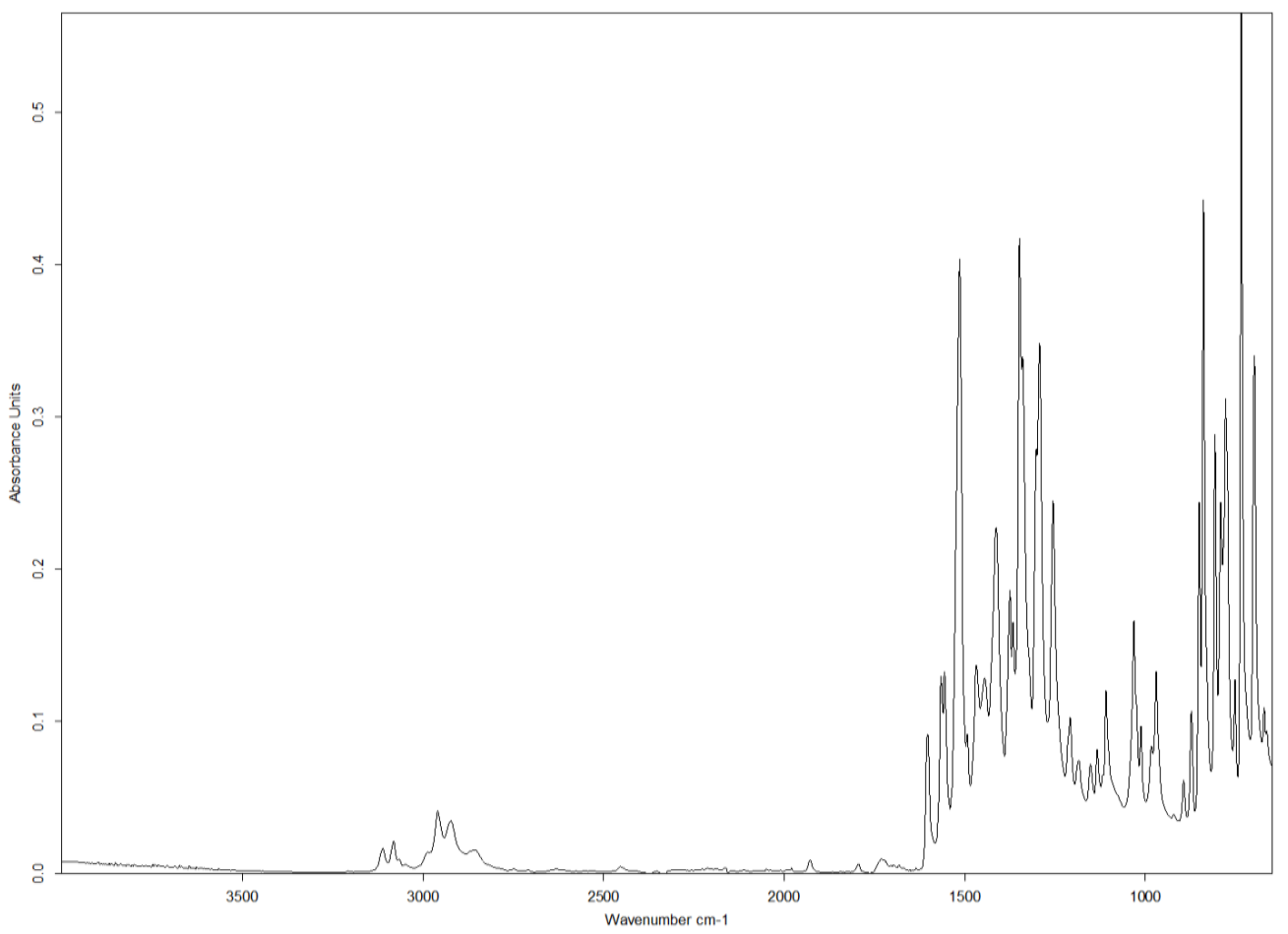

Figure S3.36 IR (Diamond ATR) spectrum of 4-bis(3,5-dimethylpyrazol-1-yl)methylnitrobenzene.<smiles>Cc1cc(C)n(C(=O)n2nc(C)cc2C)n1</smiles><smiles>Cc1cc(C)n(C(c2ccc(F)cc2)n2nc(C)cc2C)n1</smiles>

\section{4-bis(3,5-dimethylpyrazol-1-yl)methyl-fluorobenzene.}




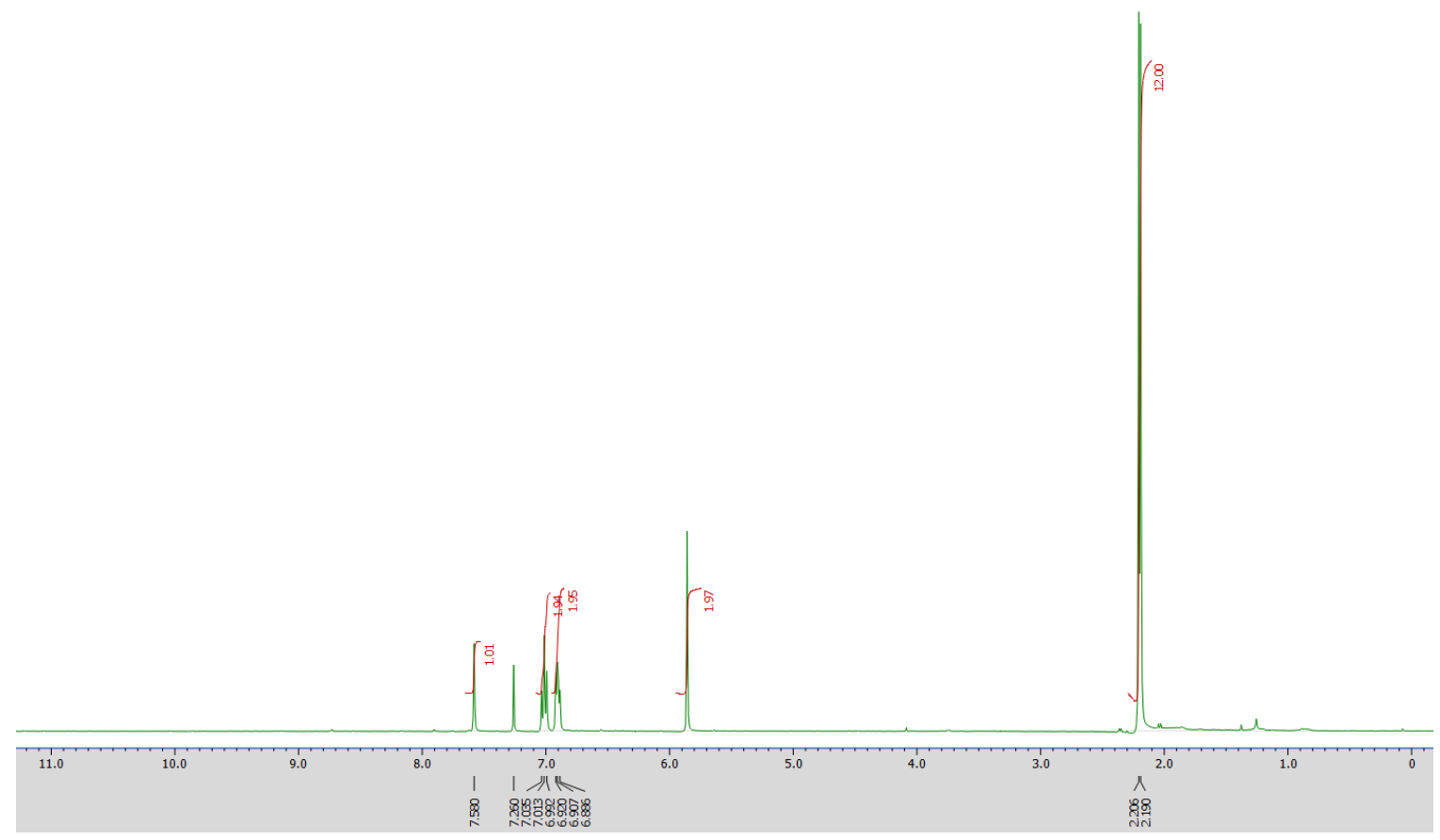

Figure $\mathrm{S} 3.37{ }^{1} \mathrm{H}$ NMR (400 MHz, $\left.\mathrm{CDCl}_{3}\right)$ spectrum of 4-bis(3,5-dimethylpyrazol-1yl)methyl-fluorobenzene

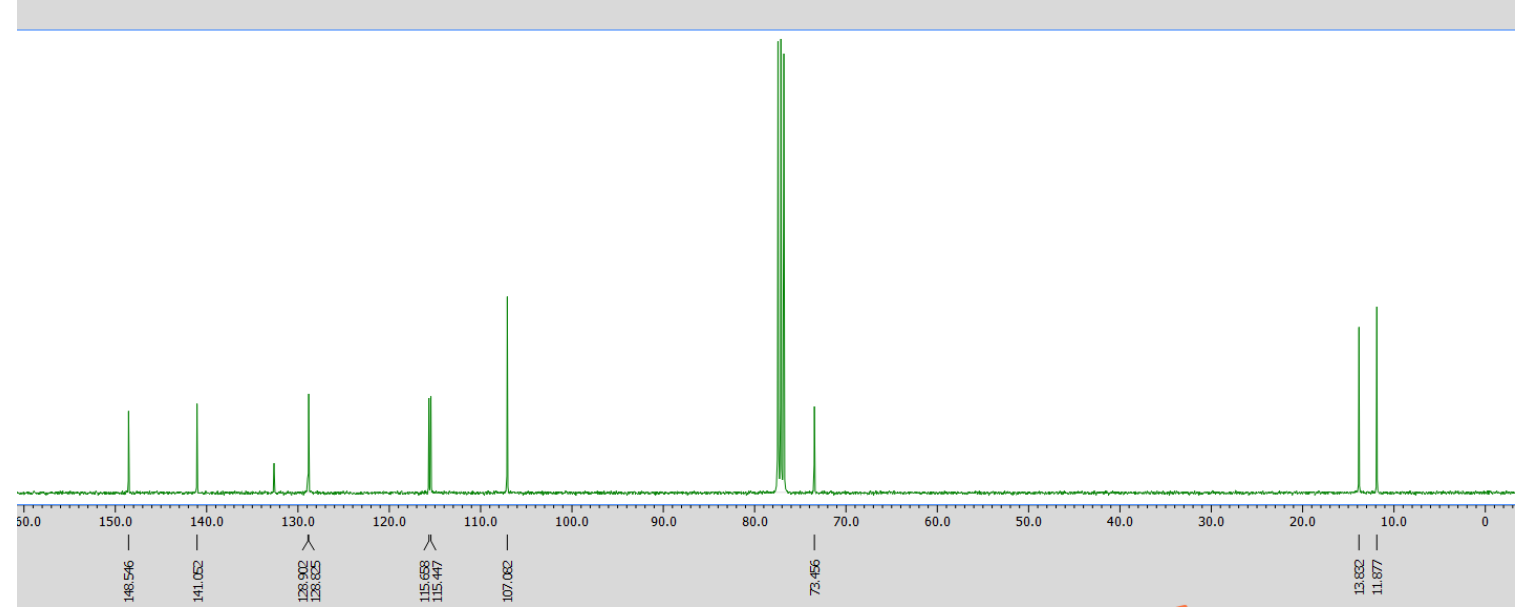

Figure $\mathrm{S} 3.38{ }^{13} \mathrm{C}$ NMR $\left(100 \mathrm{MHz}, \mathrm{CDCl}_{3}\right)$ spectrum of 4-bis(3,5-dimethylpyrazol-1yl)methyl-fluorobenzene 


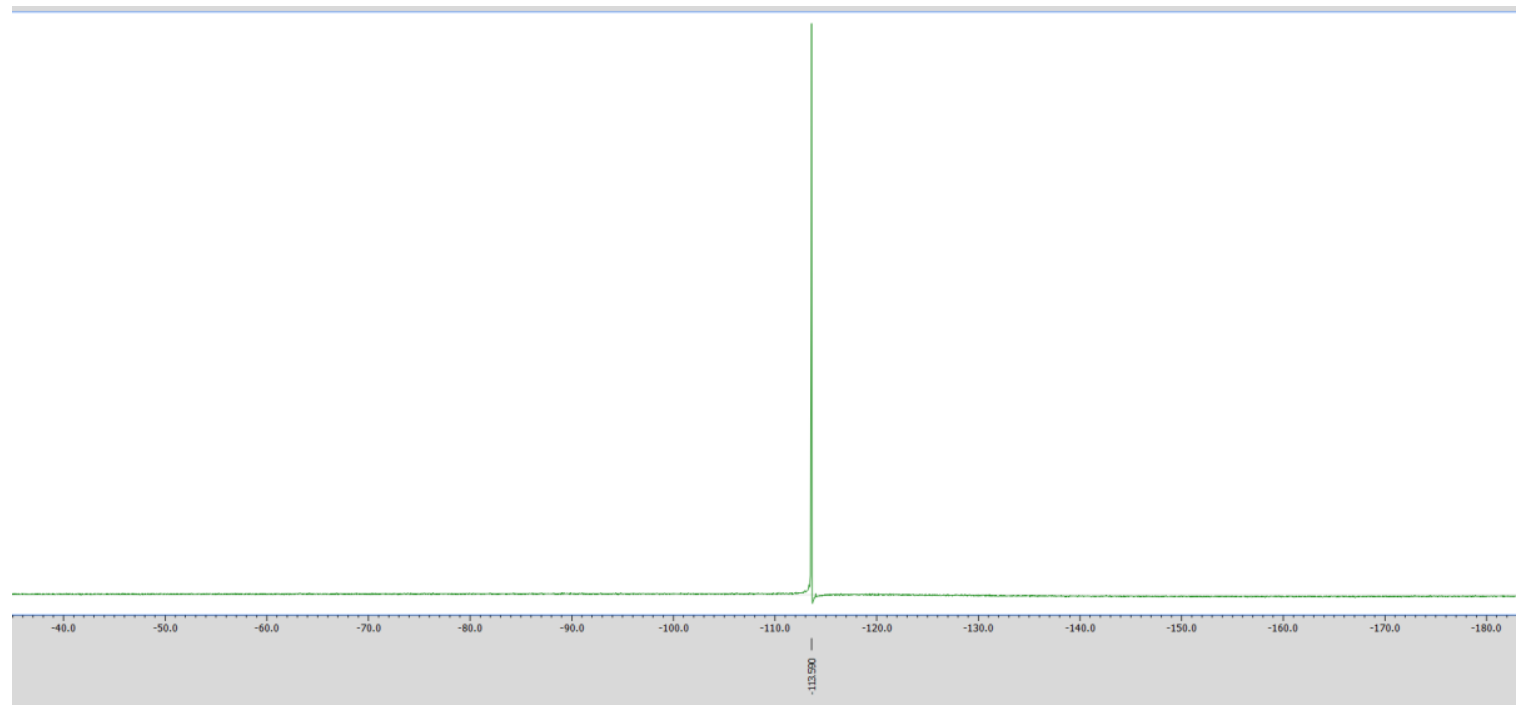

Figure $\mathrm{S} 3.39{ }^{19} \mathrm{~F}$ NMR $\left(376 \mathrm{MHz}, \mathrm{CDCl}_{3}\right)$ spectrum of 4-bis(3,5-dimethylpyrazol-1yl)methyl-fluorobenzene

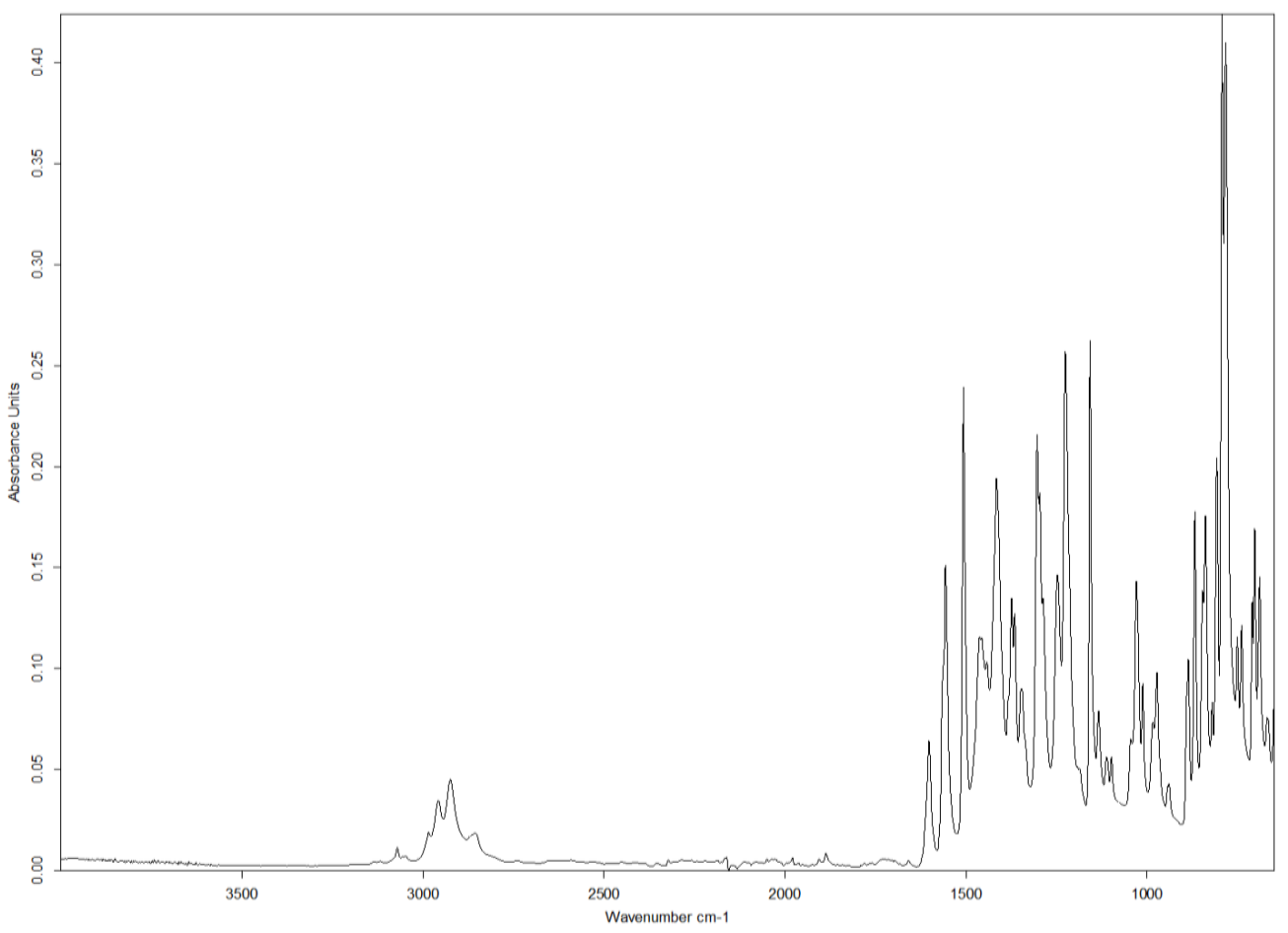

Figure S3.40 IR (Diamond ATR) spectrum of 4-bis(3,5-dimethylpyrazol-1-yl)methylfluorobenzene 


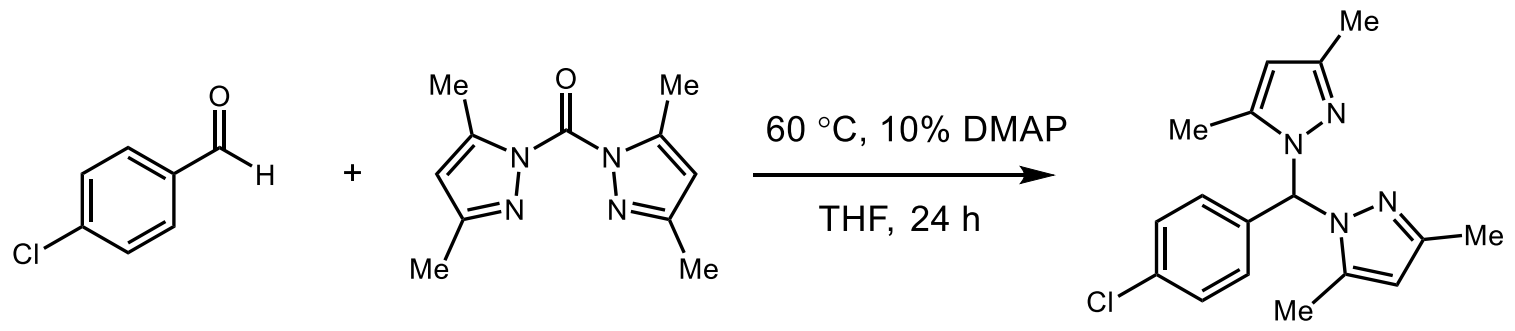

4-bis(3,5-dimethylpyrazol-1-yl)methyl-chlorobenzene.

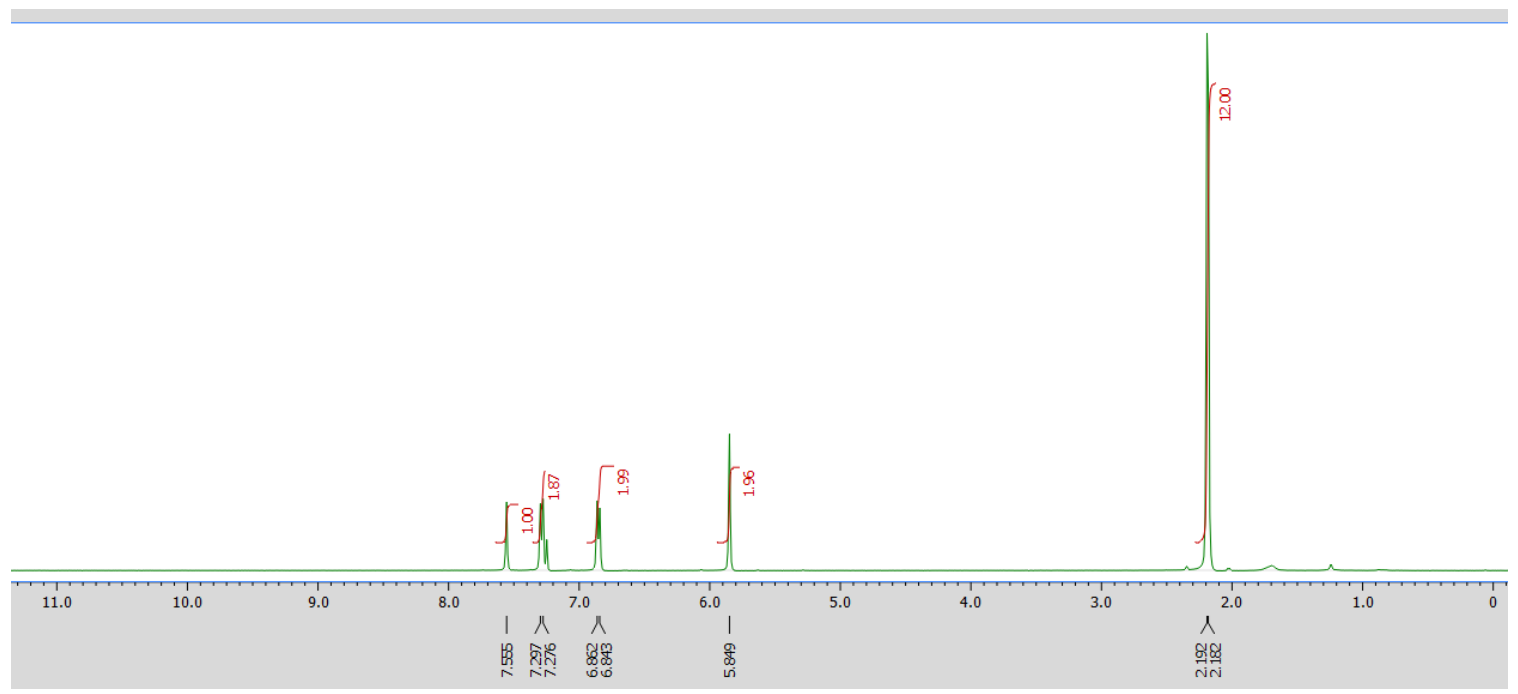

Figure $\mathrm{S} 3.41{ }^{1} \mathrm{H}$ NMR (400 $\left.\mathrm{MHz}, \mathrm{CDCl}_{3}\right)$ spectrum of 4-bis(3,5-dimethylpyrazol-1yl)methyl-chlorobenzene

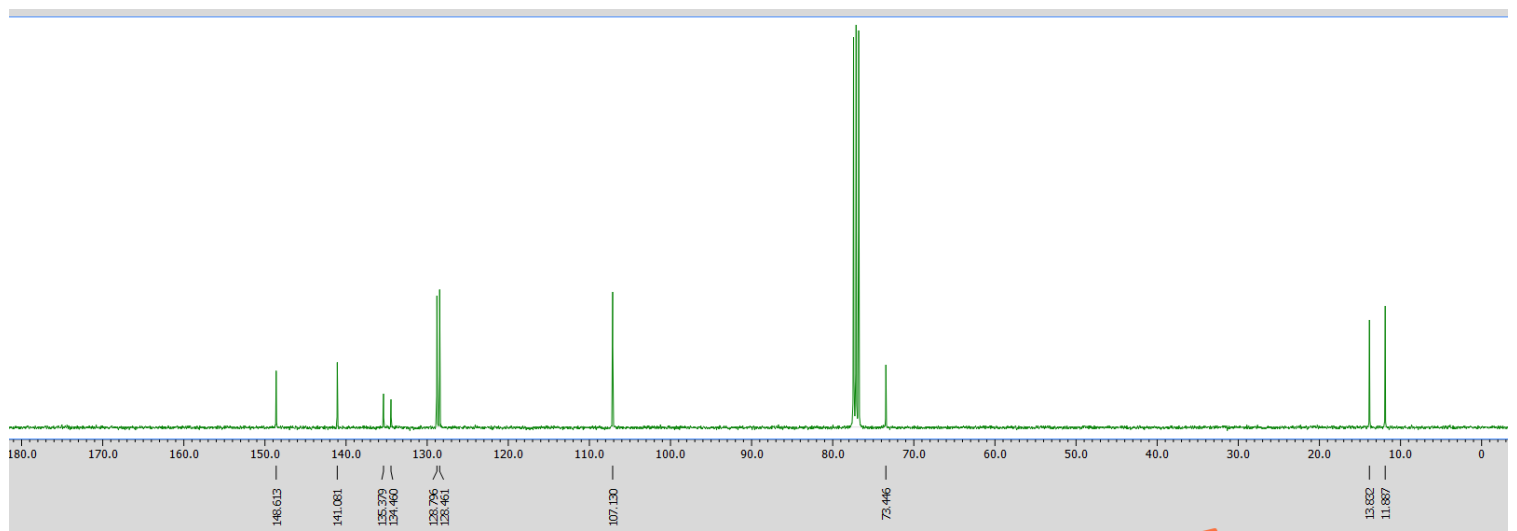

Figure $\mathrm{S} 3.42{ }^{13} \mathrm{C}$ NMR (400 $\left.\mathrm{MHz}, \mathrm{CDCl}_{3}\right)$ spectrum of 4-bis(3,5-dimethylpyrazol-1yl)methyl-chlorobenzene 


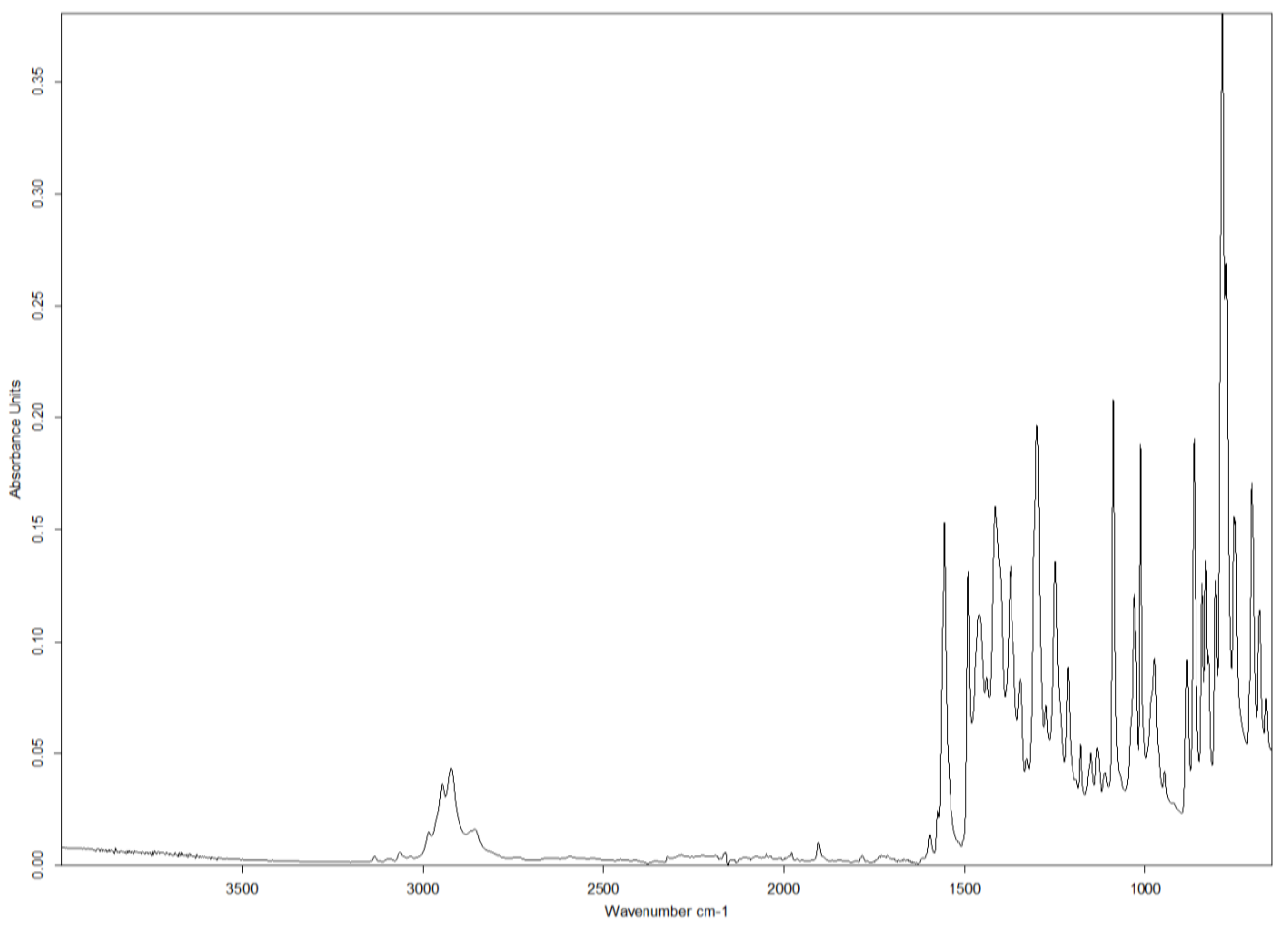

Figure S3.43 IR (Diamond ATR) spectrum of 4-bis(3,5-dimethylpyrazol-1-yl)methylchlorobenzene<smiles>O=Cc1ccccc1P</smiles><smiles>O=C(n1cccn1)n1cccn1</smiles>

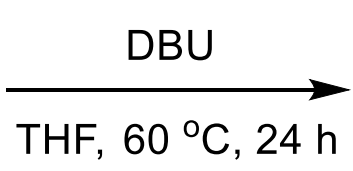<smiles>Pc1ccccc1C(n1cccn1)n1cccn1</smiles>

2-bis(pyrazol-1-yl)methyl-1-(diphenylphosphino)benzene. 


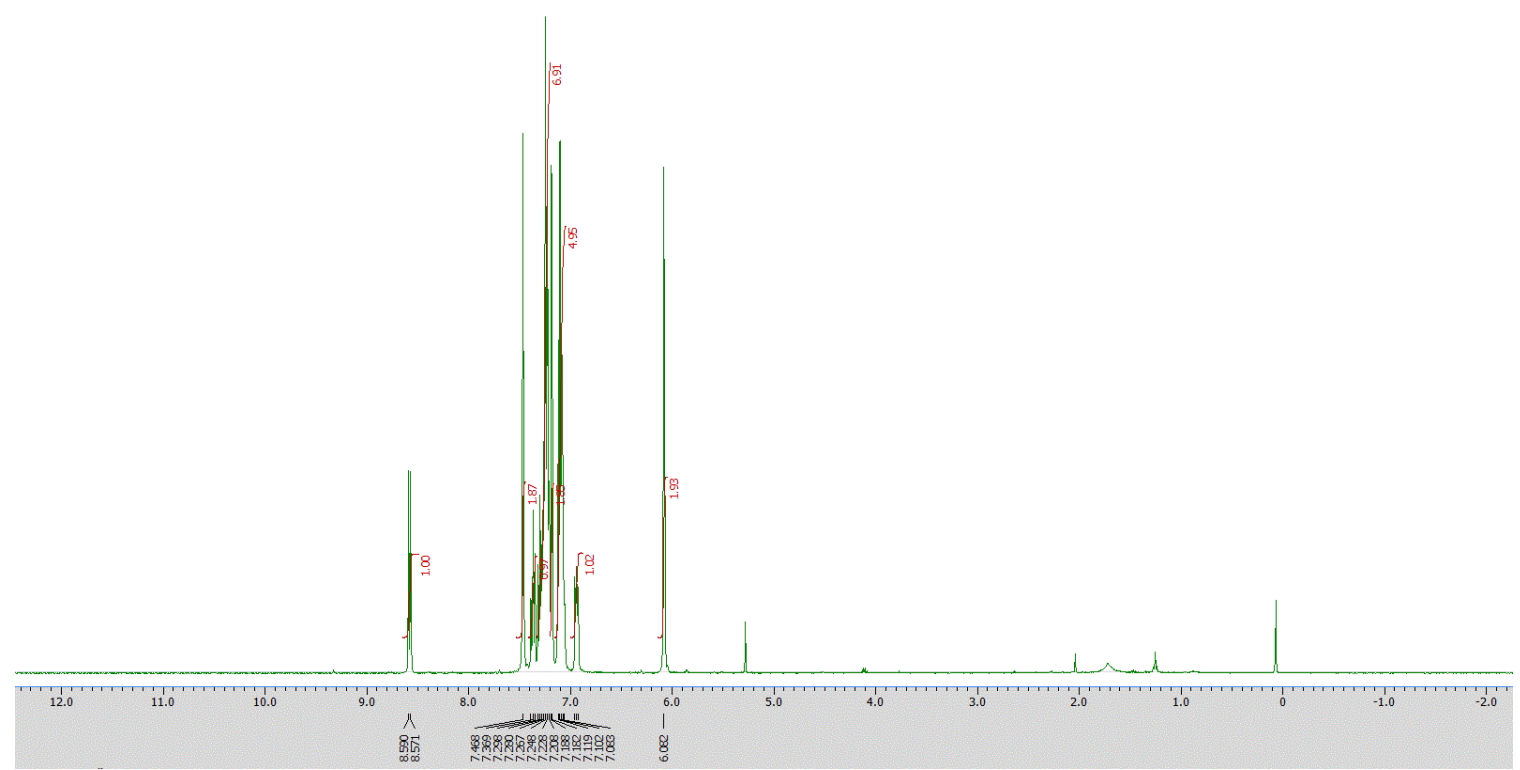

Figure $\mathrm{S} 3.44{ }^{1} \mathrm{H}$ NMR (400 $\left.\mathrm{MHz}, \mathrm{CDCl}_{3}\right)$ spectrum of 2-bis(pyrazol-1-yl)methyl-1(diphenylphosphino)benzene

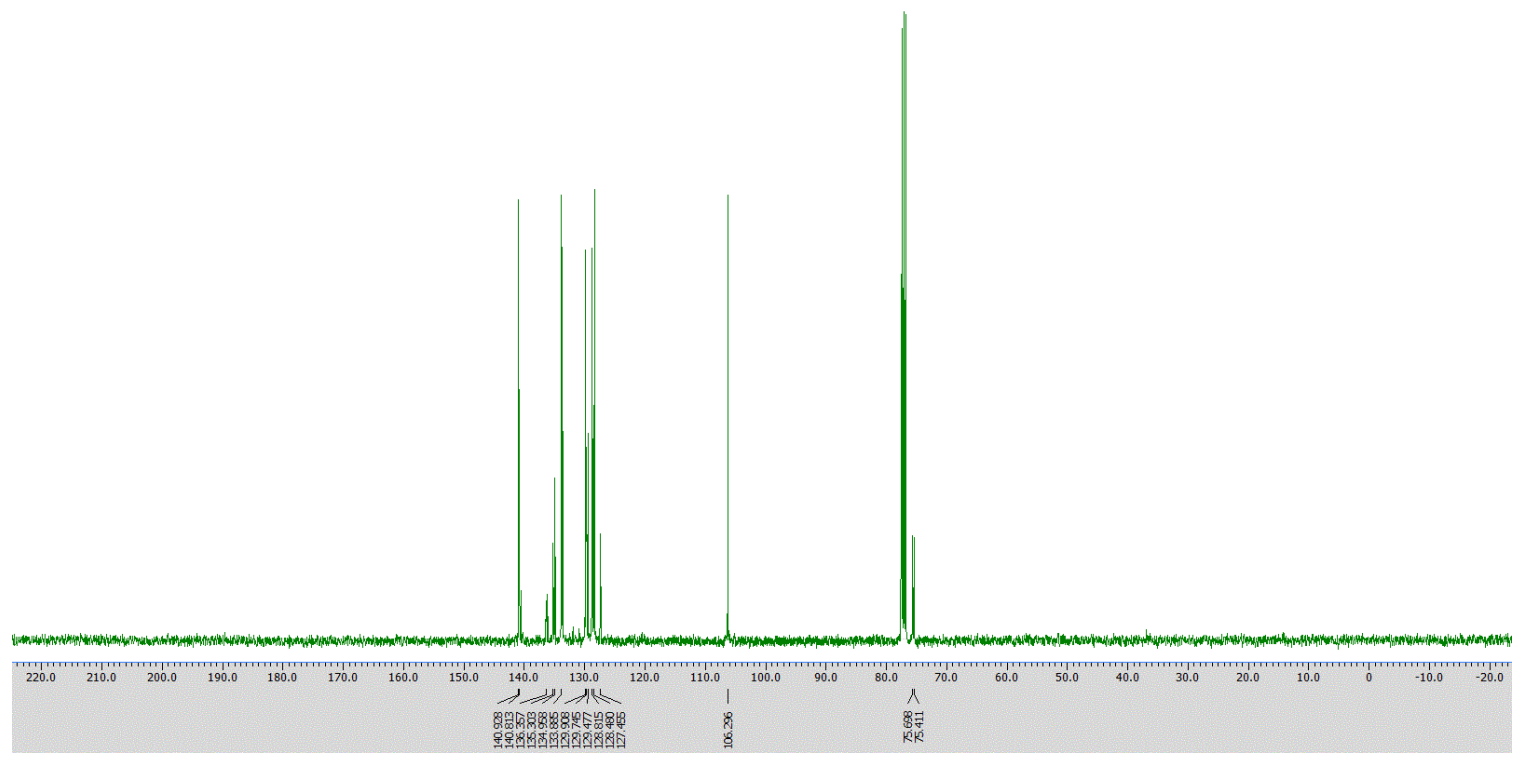

Figure $\mathrm{S} 3.45{ }^{13} \mathrm{C}$ NMR (101 $\left.\mathrm{MHz}, \mathrm{CDCl}_{3}\right)$ spectrum of 2-bis(pyrazol-1-yl)methyl-1(diphenylphosphino)benzene 


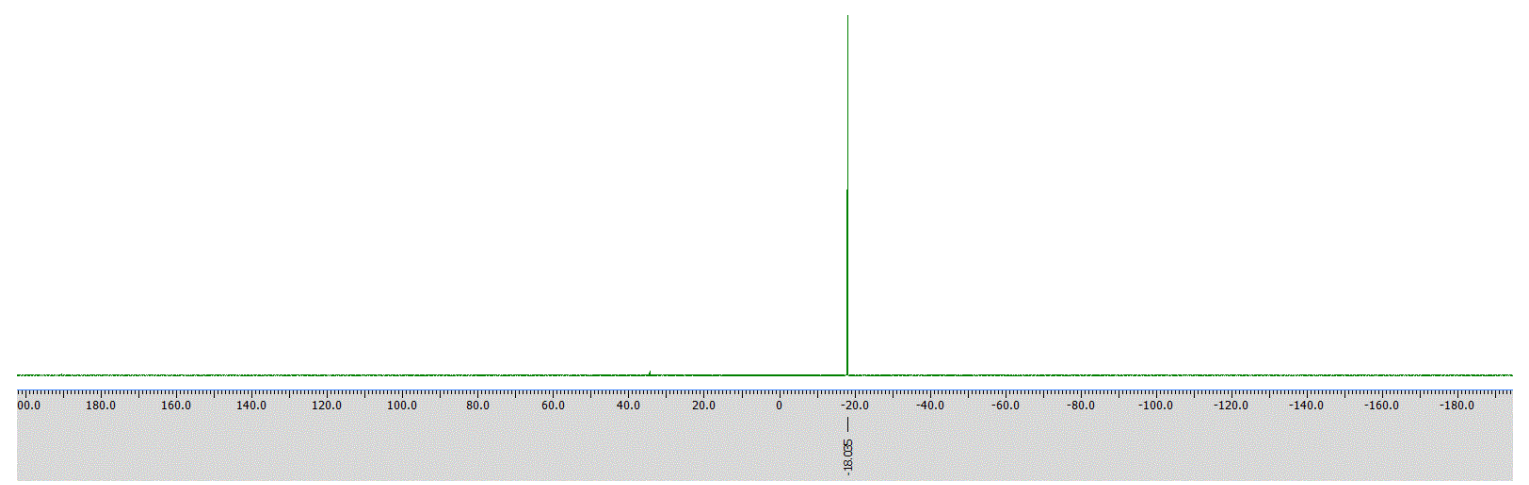

Figure S3.46 ${ }^{31} \mathrm{P}$ NMR (162 MHz, $\left.\mathrm{CDCl}_{3}\right)$ spectrum of 2-bis(pyrazol-1-yl)methyl-1(diphenylphosphino)benzene

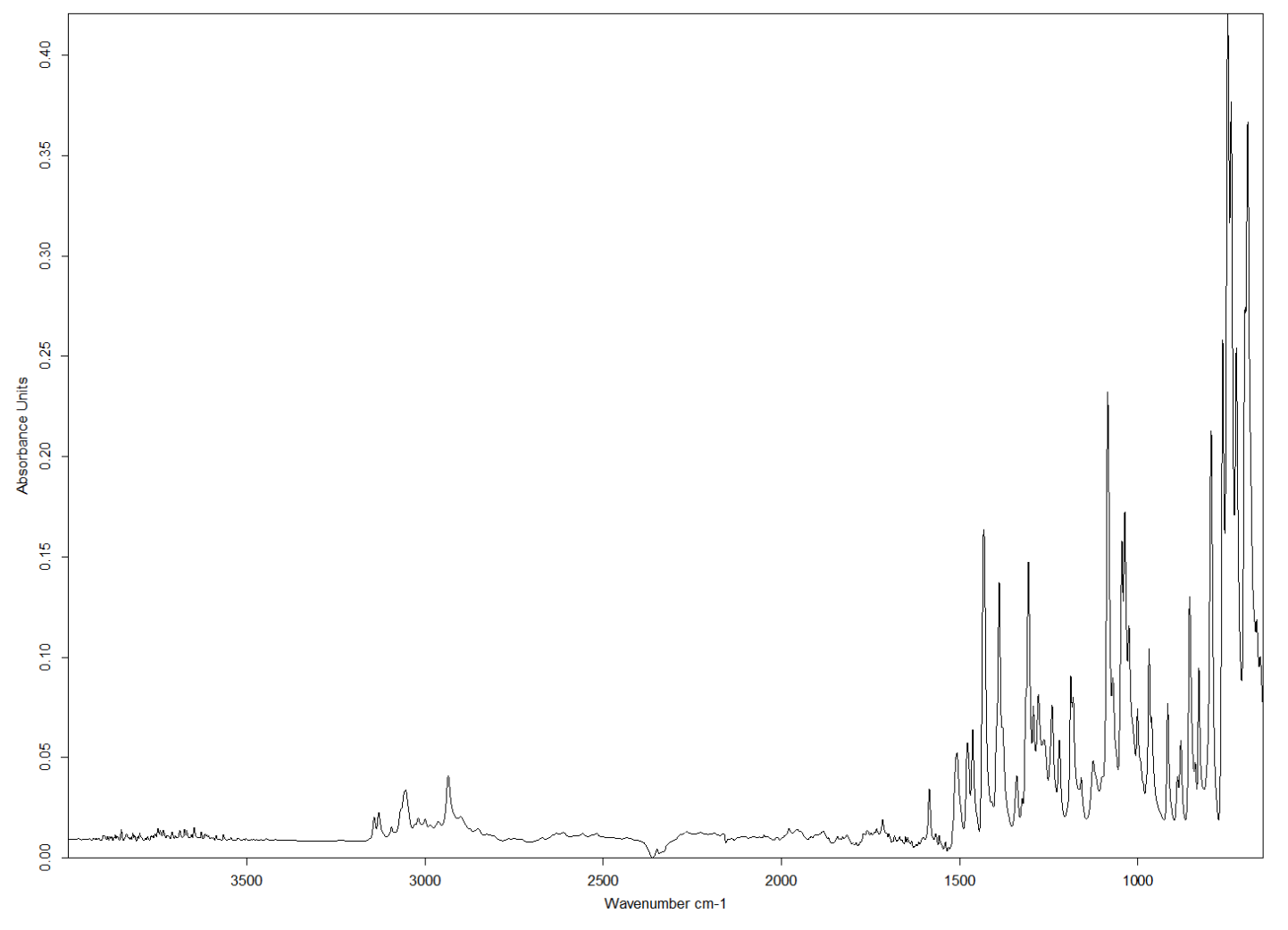


Figure S3.47 IR (Diamond ATR) spectrum of 2-bis(pyrazol-1-yl)methyl-1(diphenylphosphino)benzene<smiles>O=Cc1nccn1Cc1ccccc1</smiles><smiles>Cc1cc(C)n(C(=O)n2nc(C)cc2C)n1</smiles>

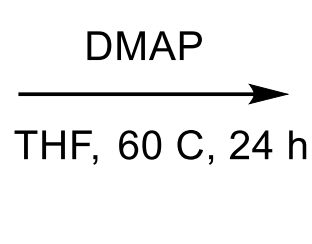<smiles>Cc1cc(C)n(C(c2nccn2Cc2ccccc2)n2nc(C)cc2C)n1</smiles>

$N$-benzyl-2-bis(3,5-dimethylpyrazol-1-yl)methylimidazole.

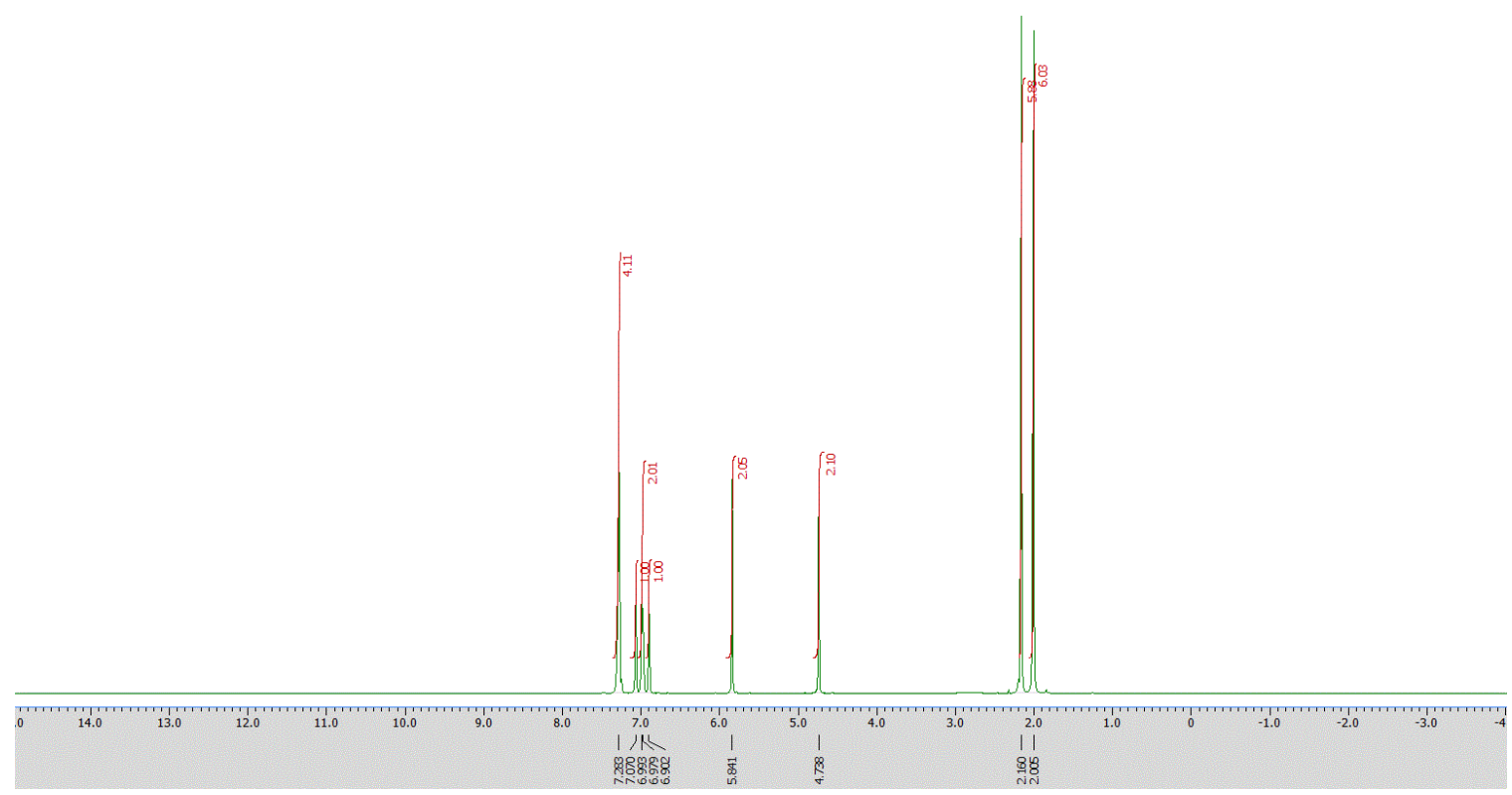

Figure $\mathrm{S} 3.48{ }^{1} \mathrm{H} \quad \mathrm{NMR} \quad\left(400 \mathrm{MHz}, \mathrm{CDCl}_{3}\right)$ spectrum of $\boldsymbol{N}$-benzyl-2-bis(3,5dimethylpyrazol-1-yl)methylimidazole 


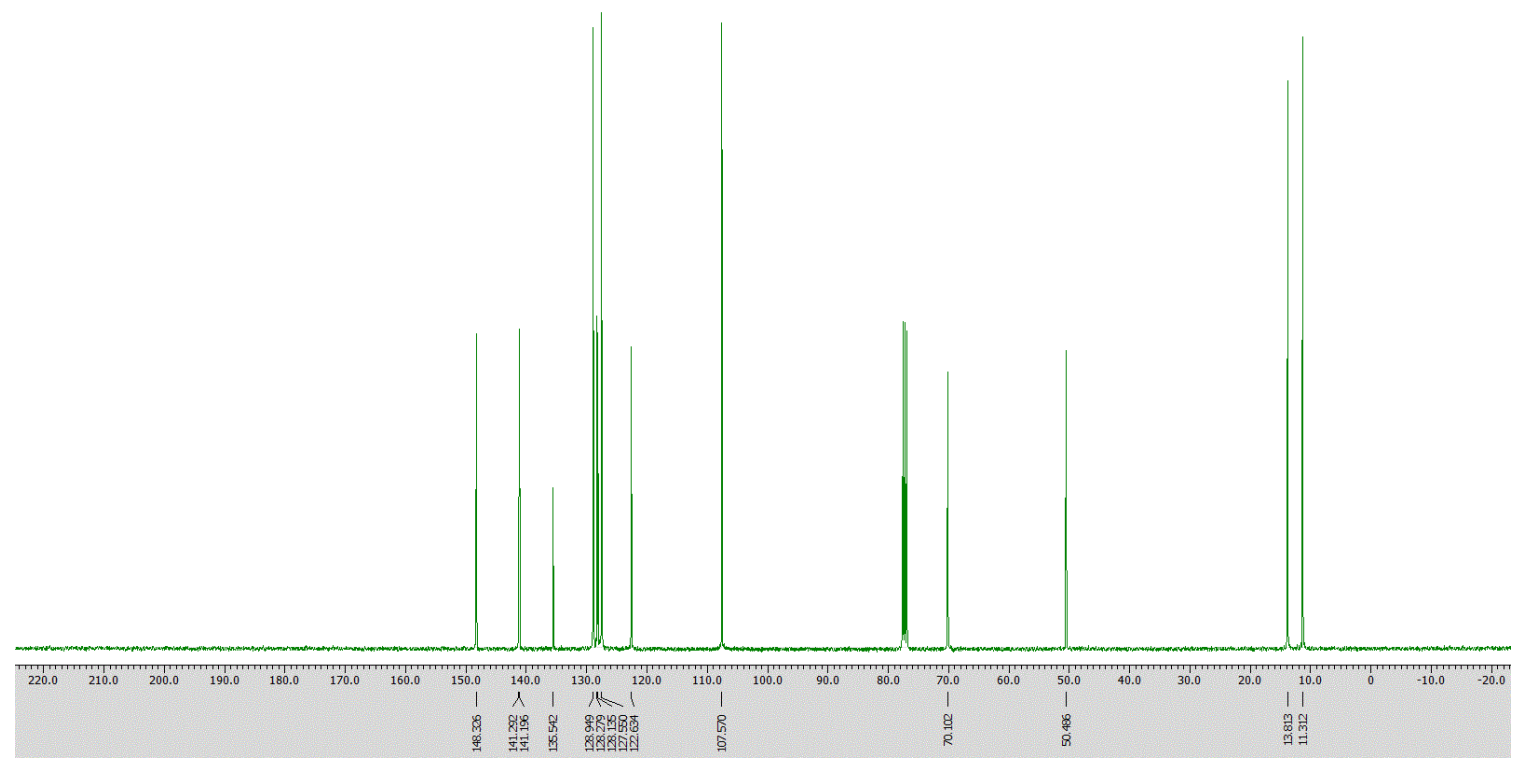

Figure $\mathrm{S} 3.49{ }^{13} \mathrm{C} \quad \mathrm{NMR} \quad\left(101 \mathrm{MHz}, \mathrm{CDCl}_{3}\right)$ spectrum of $\boldsymbol{N}$-benzyl-2-bis(3,5dimethylpyrazol-1-yl)methylimidazole

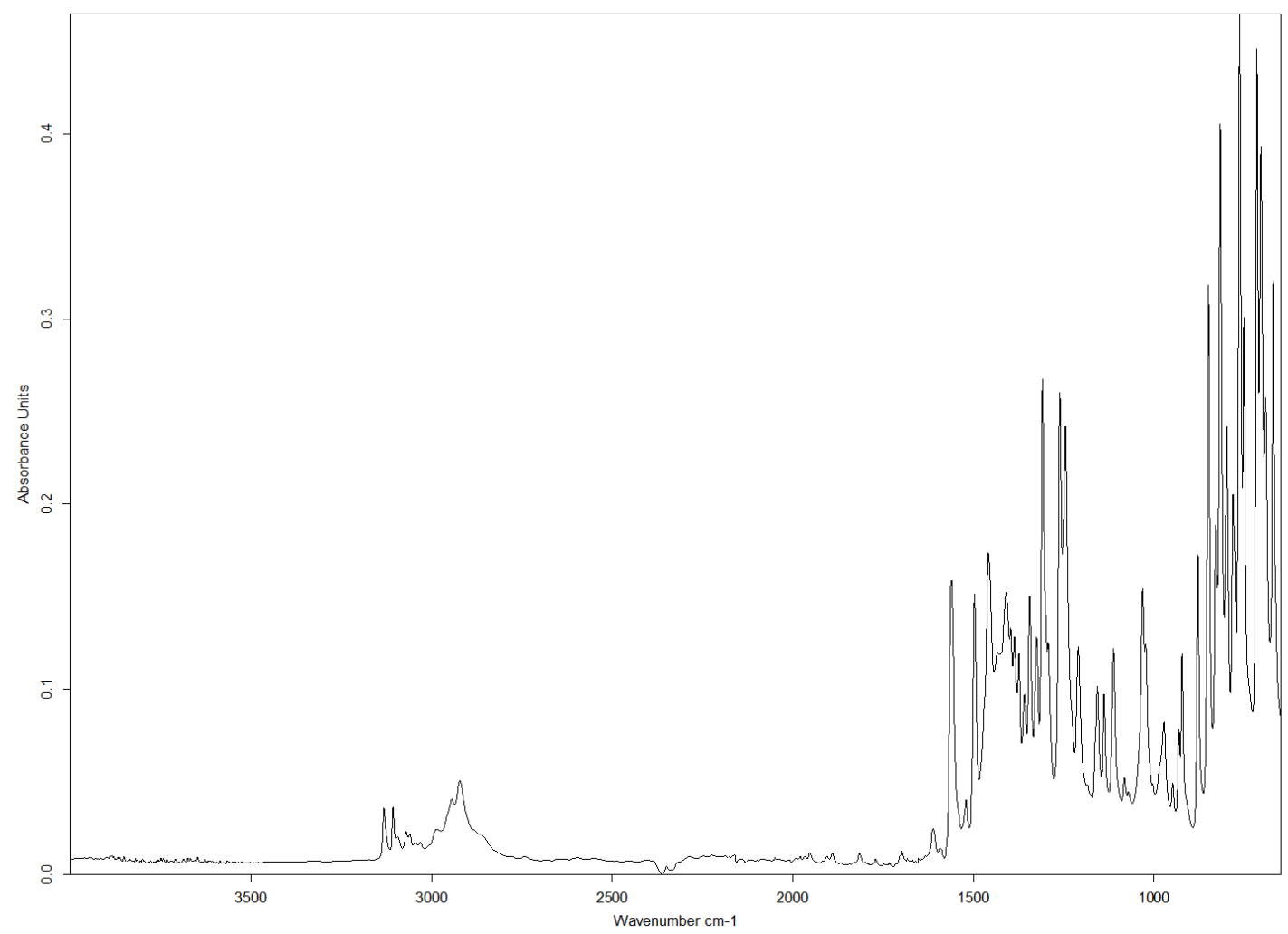


Figure S3.50 IR (Diamond ATR) spectrum of $N$-benzyl-2-bis(3,5-dimethylpyrazol-1yl)methylimidazole

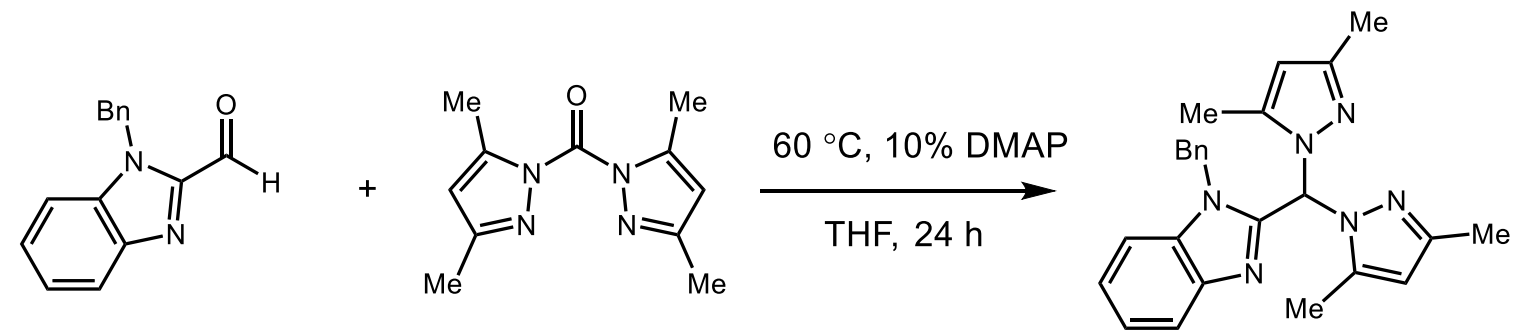

2-bis(3,5-dimethylpyrazol-1-yl)methyl- $N$-benzyl-benzimidazole.

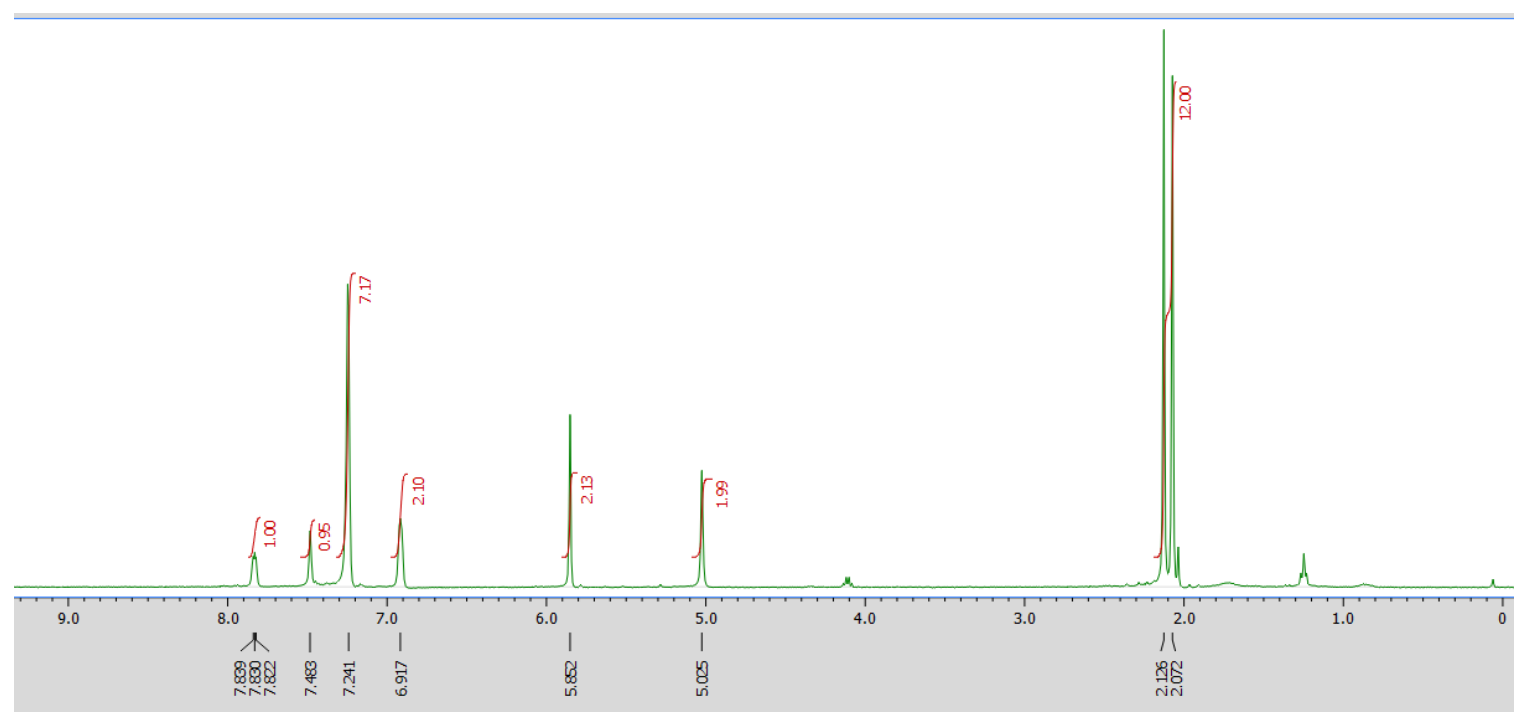

Figure $\mathrm{S} 3.51{ }^{1} \mathrm{H}$ NMR $\left(400 \mathrm{MHz}, \mathrm{CDCl}_{3}\right)$ spectrum of 2-bis(3,5-dimethylpyrazol-1yl)methyl- $N$-benzyl-benzimidazole 


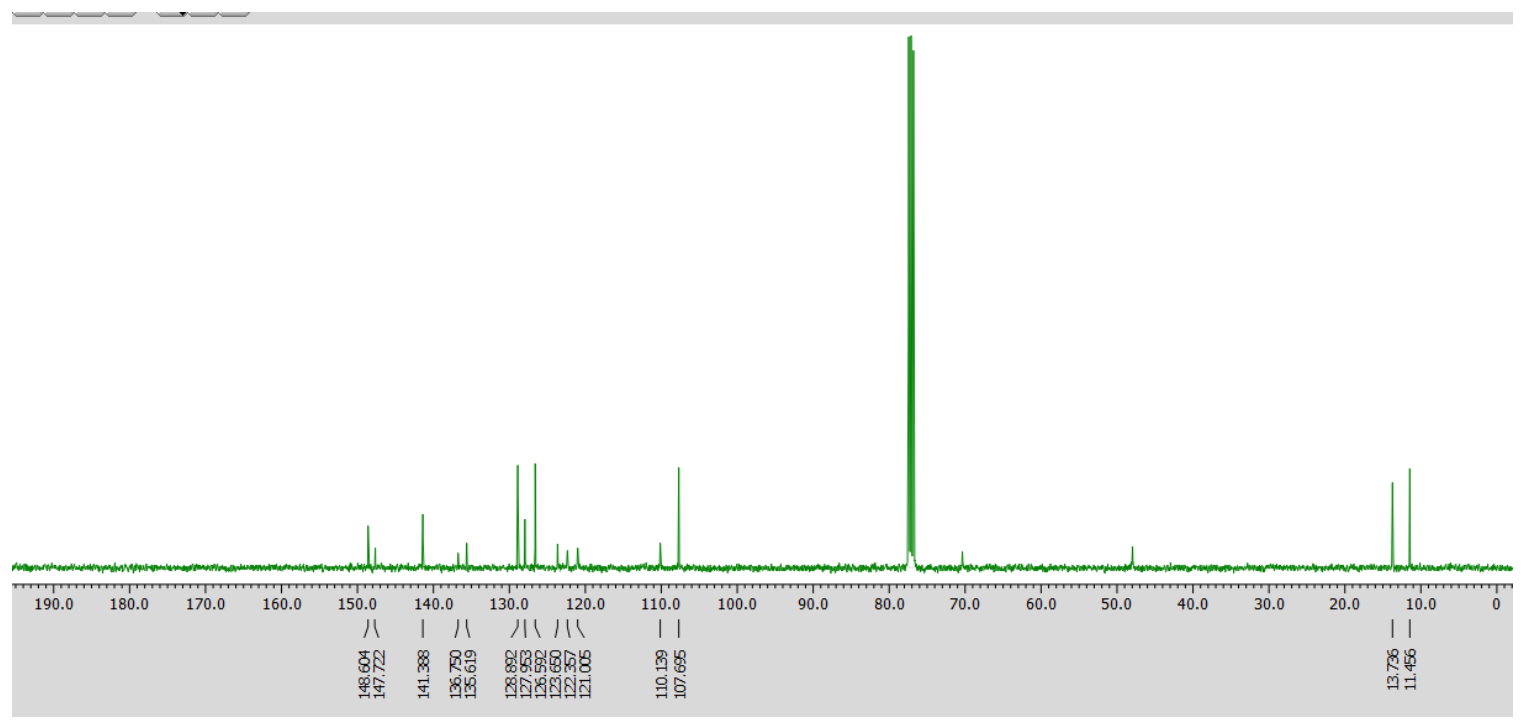

Figure $\mathrm{S} 3.52{ }^{13} \mathrm{C}$ NMR (101 $\left.\mathrm{MHz}, \mathrm{CDCl}_{3}\right)$ spectrum of 2-bis(3,5-dimethylpyrazol-1yl)methyl- $N$-benzyl-benzimidazole

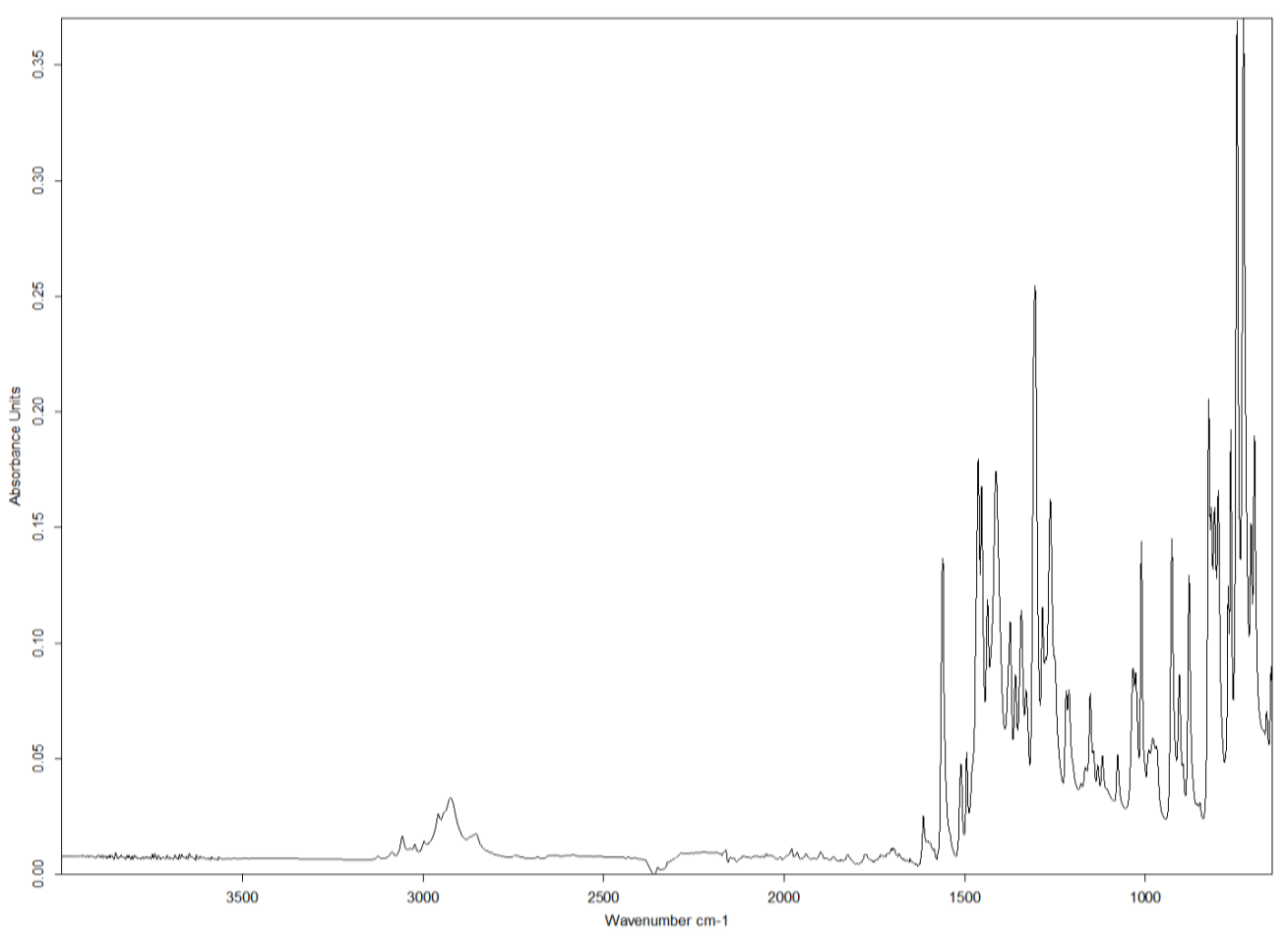

Figure S3.53 IR (Diamond ATR) spectrum of 2-bis(3,5-dimethylpyrazol-1-yl)methyl- $N$ benzyl-benzimidazole 
<smiles>O=C/C=C/c1ccccc1</smiles><smiles>Cc1cc(C)n(C(=O)n2nc(C)cc2C)n1</smiles><smiles>Cc1cc(C)n(C(/C=C/c2ccccc2)n2nc(C)cc2C)n1</smiles>

(E)-3,3-bis(3,5-dimethylpyrazol-1-yl)-1-phenylpropene.

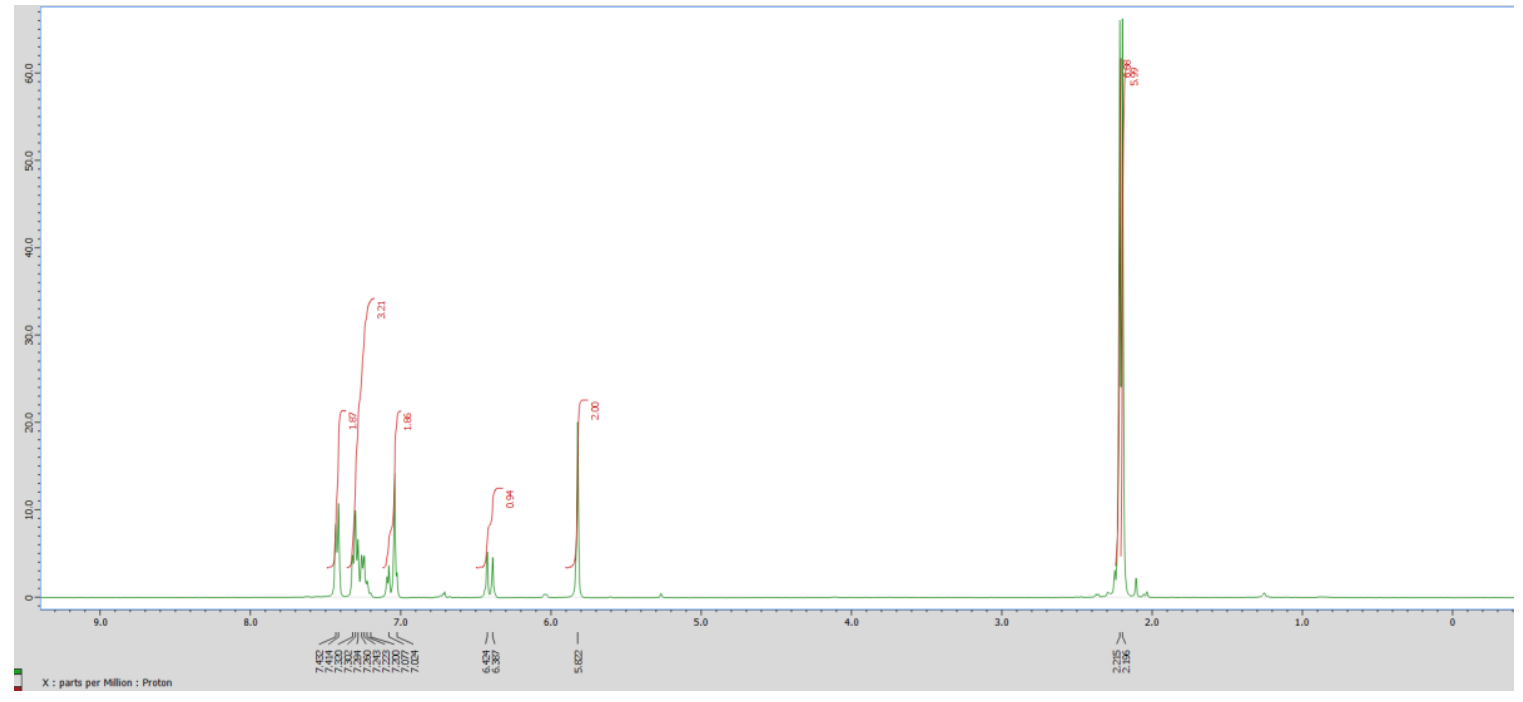

Figure S3.54 ${ }^{1} \mathrm{H}$ NMR $\left(400 \mathrm{MHz}, \mathrm{CDCl}_{3}\right)$ spectrum of (E)-3,3-bis(3,5-dimethylpyrazol1-yl)-1-phenylpropene 


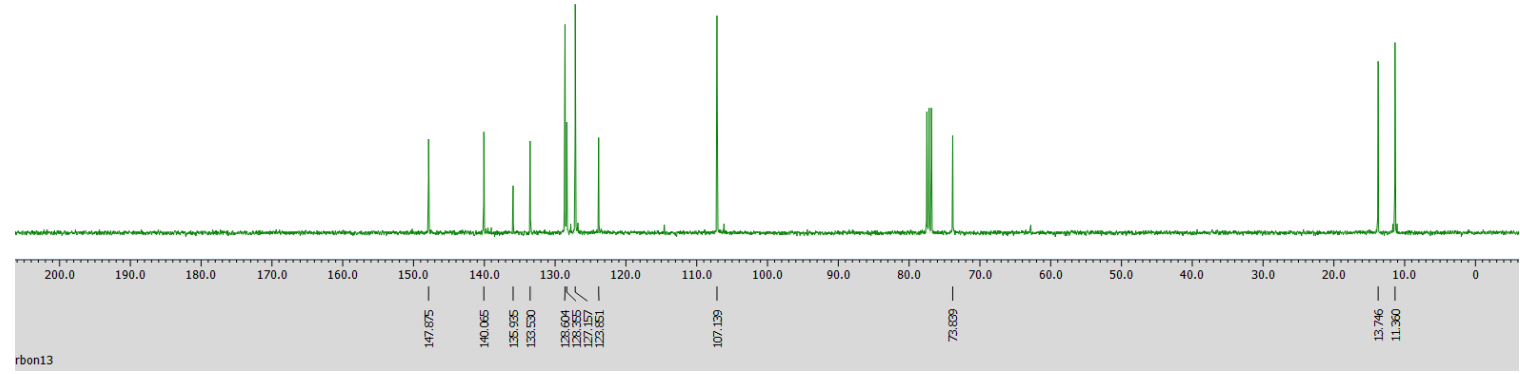

Figure $\mathrm{S} 3.55{ }^{13} \mathrm{C}$ NMR $\left(100 \mathrm{MHz}, \mathrm{CDCl}_{3}\right)$ spectrum of (E)-3,3-bis(3,5-dimethylpyrazol1-yl)-1-phenylpropene

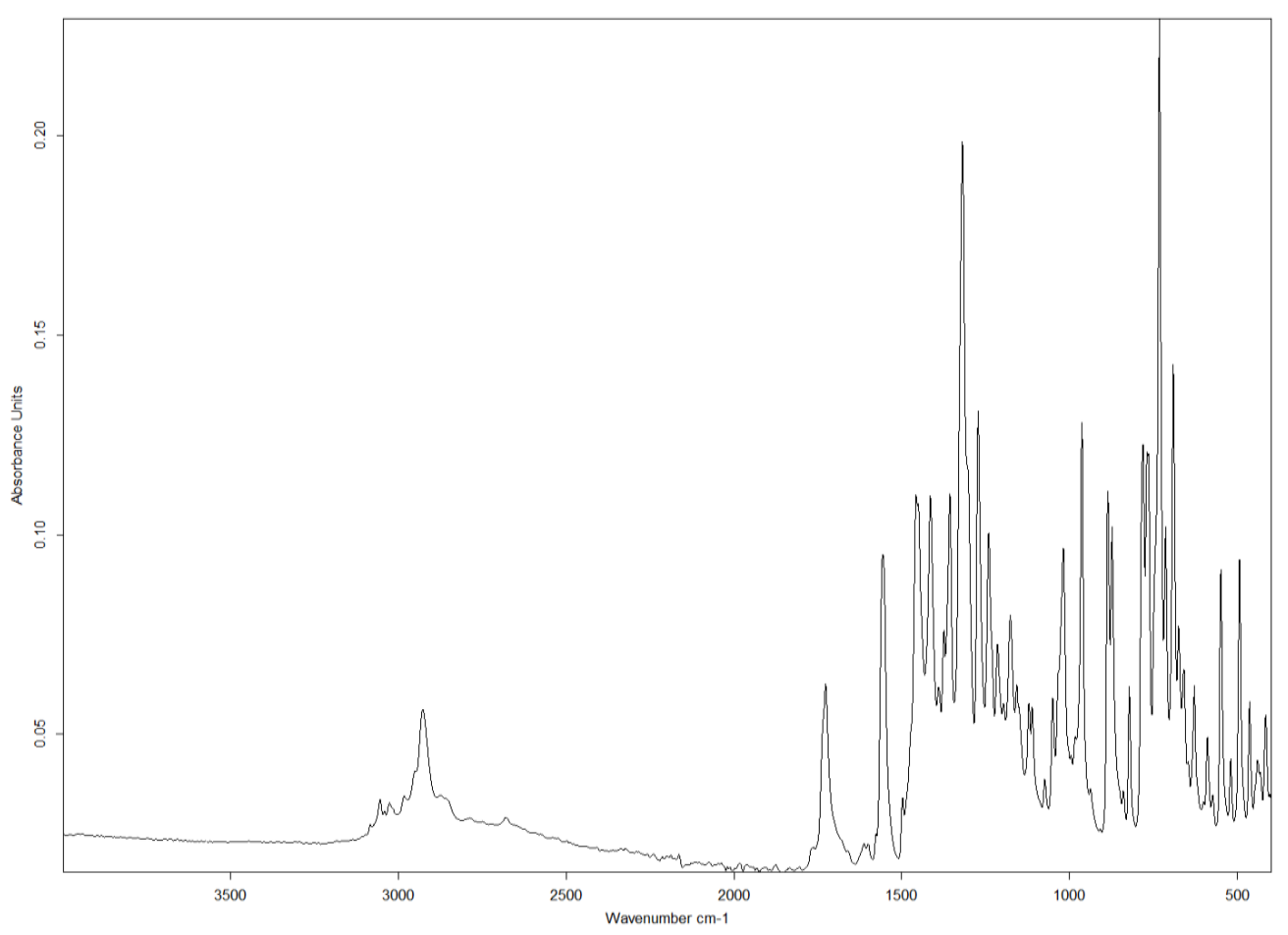


Figure S3.56 IR (Diamond ATR) spectrum of (E)-3,3-bis(3,5-dimethylpyrazol-1-yl)-1phenylpropene<smiles>O=Cc1ccco1</smiles><smiles>Cc1cc(C)n(C(=O)n2nc(C)cc2C)n1</smiles>

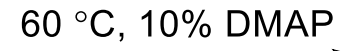
THF, $24 \mathrm{~h}$

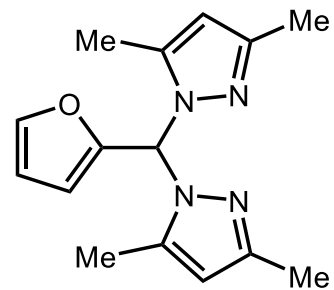

1,1'-(furan-2-ylmethylene)bis(3,5-dimethylpyrazole).

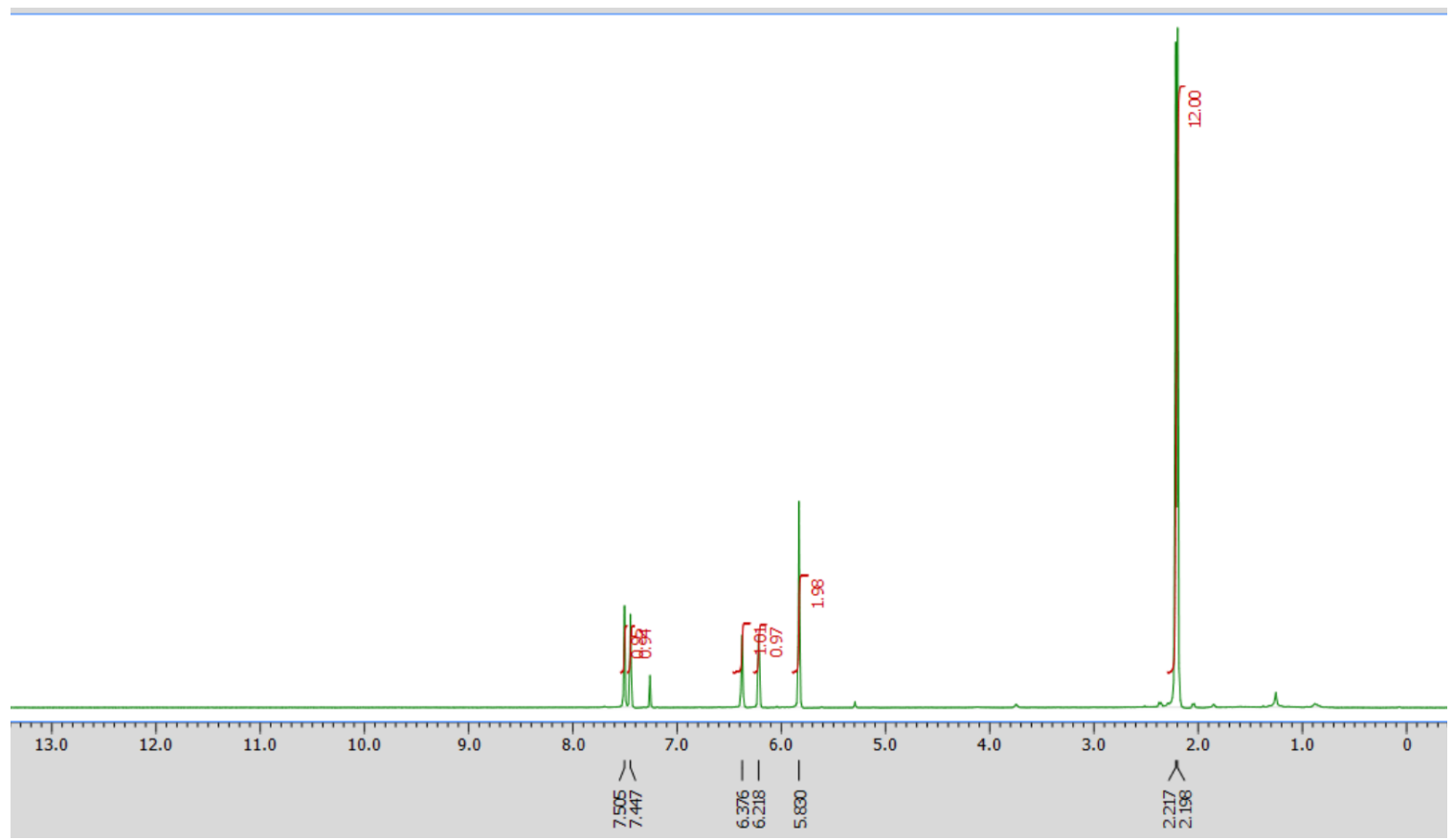

Figure $\mathrm{S} 3.57{ }^{1} \mathrm{H}$ NMR $\left(400 \mathrm{MHz}, \mathrm{CDCl}_{3}\right)$ spectrum of 1,1'-(furan-2-ylmethylene)bis(3,5dimethylpyrazole) 


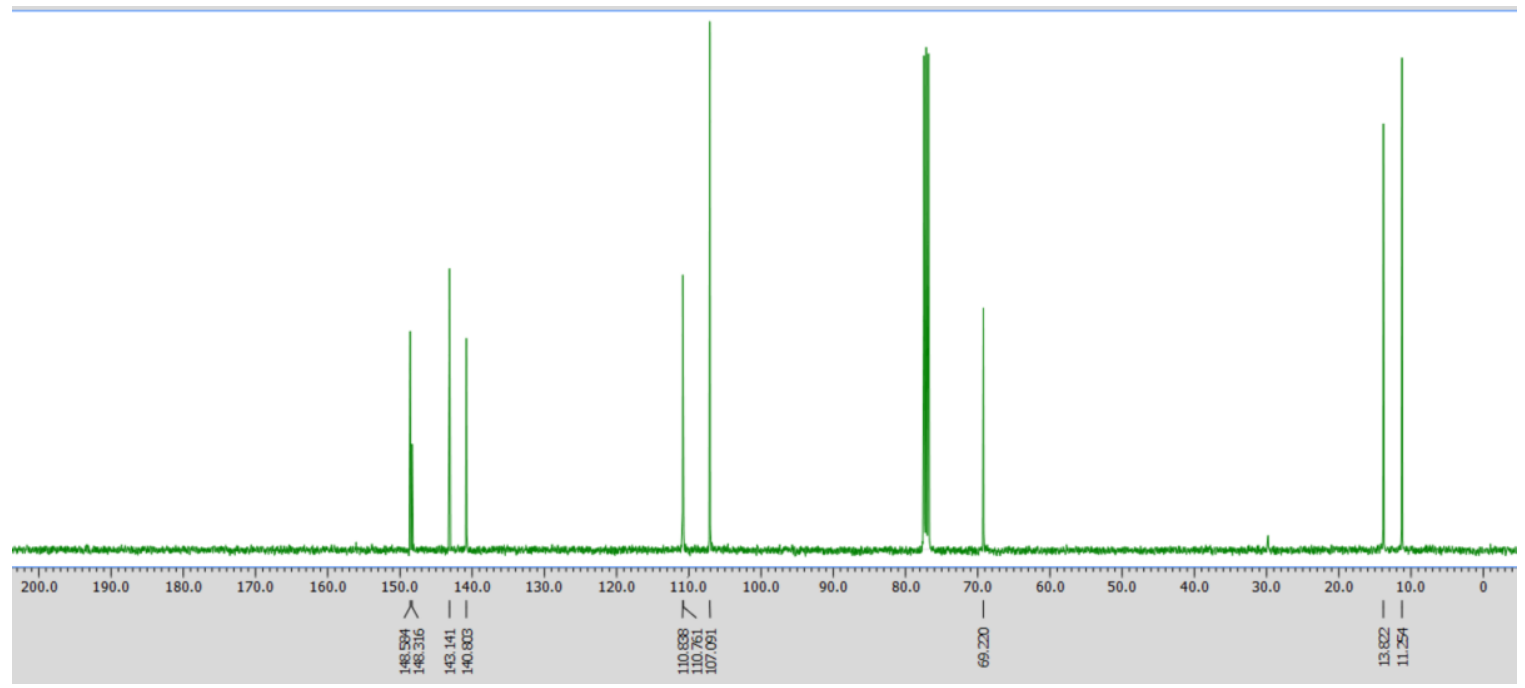

Figure $\mathrm{S} 3.58 \quad{ }^{13} \mathrm{C} \quad \mathrm{NMR} \quad\left(101 \quad \mathrm{MHz}, \mathrm{CDCl}_{3}\right)$ spectrum of 1,1'-(furan-2ylmethylene)bis(3,5-dimethylpyrazole)<smiles>O=Cc1cc2ccccc2[nH]1</smiles><smiles>Cc1cc(C)n(C(=O)n2nc(C)cc2C)n1</smiles>

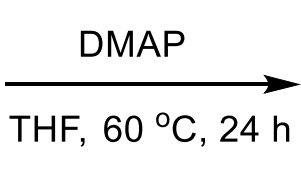<smiles>Cc1cc(C)n(C(c2cc3ccccc3[nH]2)n2nc(C)cc2C)n1</smiles>

indole-2-bis(3,5-dimethylpyrazol-1-yl)methane.

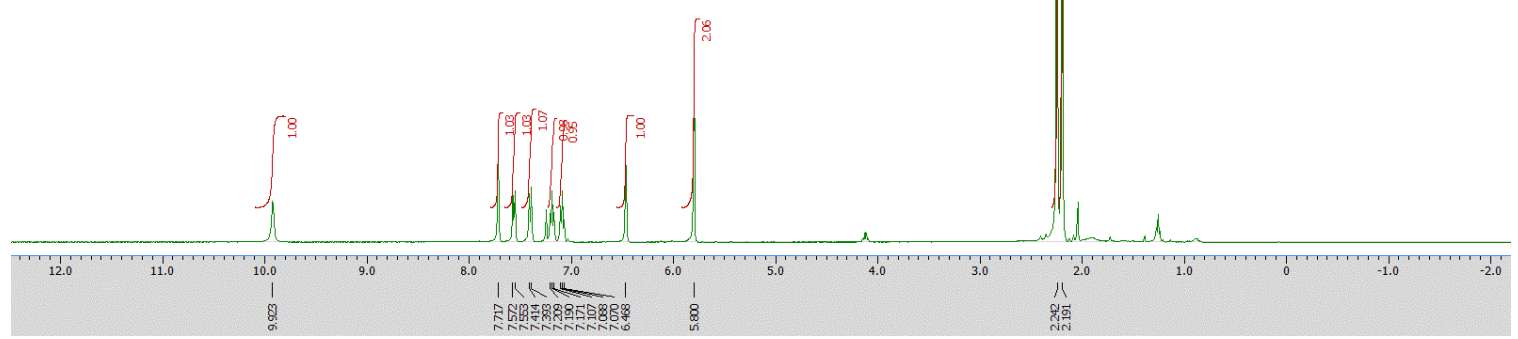


Figure $\mathrm{S} 3.59{ }^{1} \mathrm{H} \mathrm{NMR}\left(400 \mathrm{MHz}, \mathrm{CDCl}_{3}\right)$ spectrum of indole-2-bis(3,5-dimethylpyrazol1-yl)methane

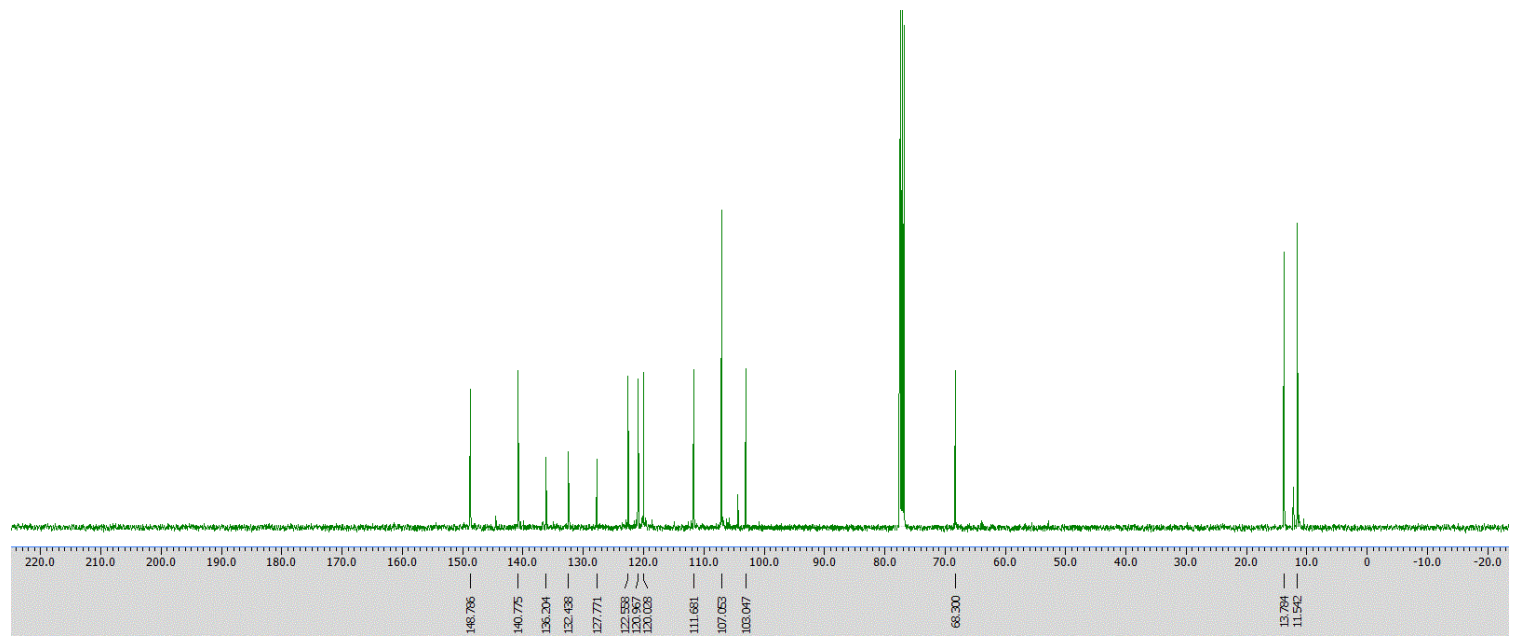

Figure $\mathrm{S} 3.60 \quad{ }^{13} \mathrm{C} \quad \mathrm{NMR} \quad\left(101 \quad \mathrm{MHz}, \mathrm{CDCl}_{3}\right)$ spectrum of indole-2-bis(3,5dimethylpyrazol-1-yl)methane 


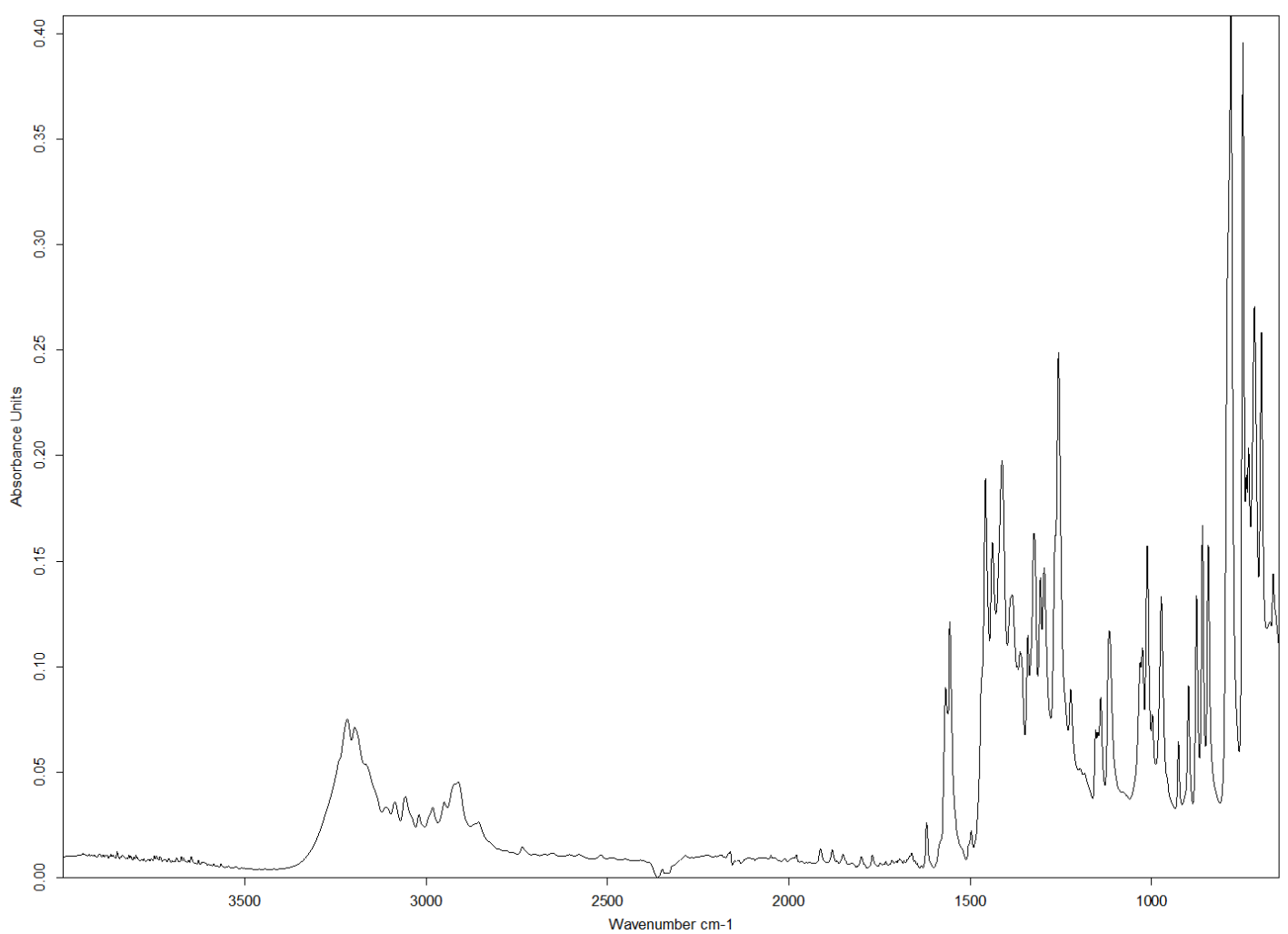

Figure S3.61 IR (Diamond ATR) spectrum of indole-2-bis(3,5-dimethylpyrazol-1yl)methane<smiles>CCC=O</smiles><smiles>Cc1cc(C)n(C(=O)n2nc(C)cc2C)n1</smiles>

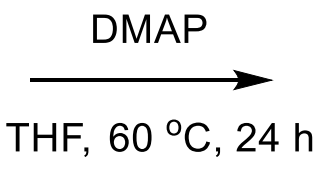<smiles>CCC(n1nc(C)cc1C)n1nc(C)cc1C</smiles>

\section{1,1-bis(3,5-dimethylpyrazol-1-yl)propane.}




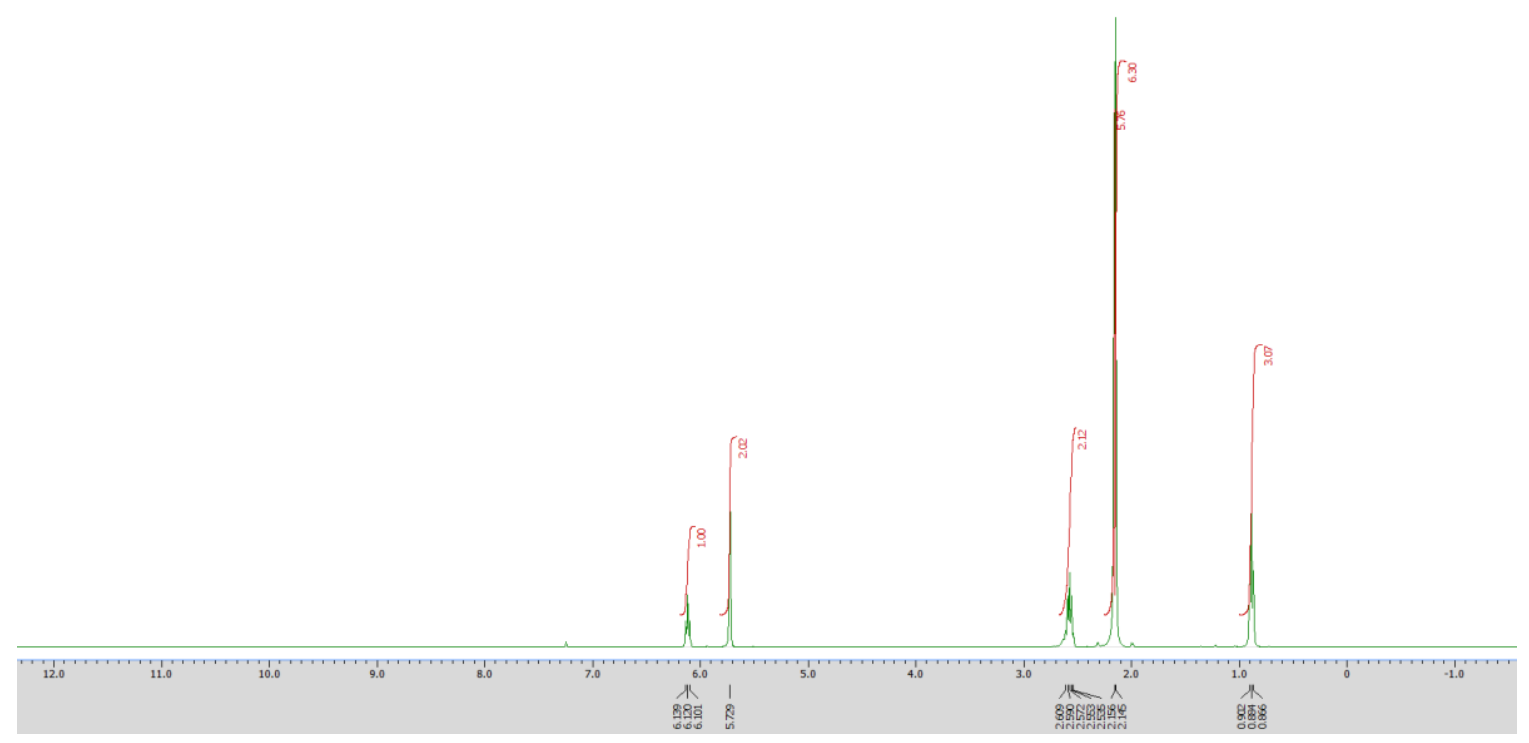

Figure S3.62 ${ }^{1} \mathrm{H}$ NMR (400 MHz, $\left.\mathrm{CDCl}_{3}\right)$ spectrum of 1,1-bis(3,5-dimethylpyrazol-1yl)propane

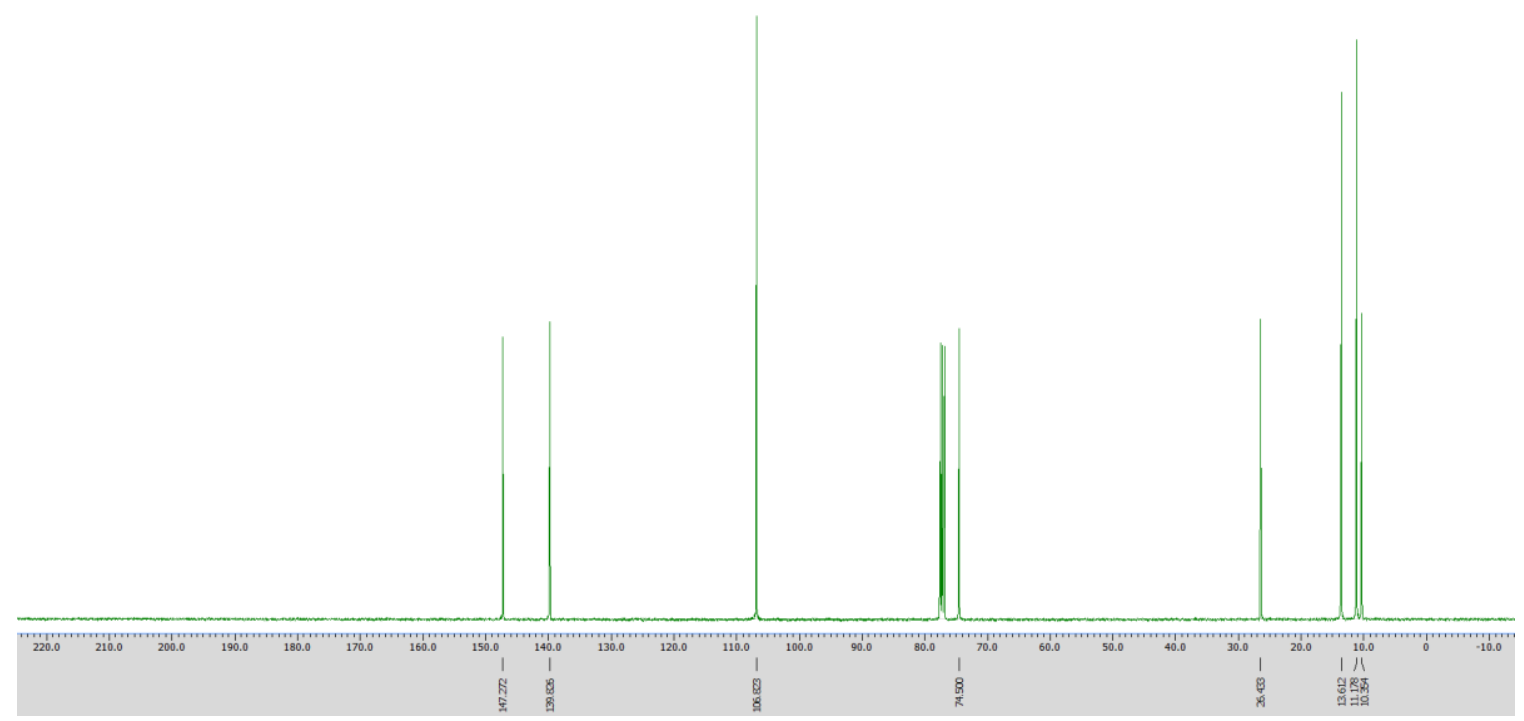

Figure $\mathrm{S} 3.63{ }^{13} \mathrm{C}$ NMR (101 MHz, $\left.\mathrm{CDCl}_{3}\right)$ spectrum of 1,1-bis(3,5-dimethylpyrazol-1yl)propane 


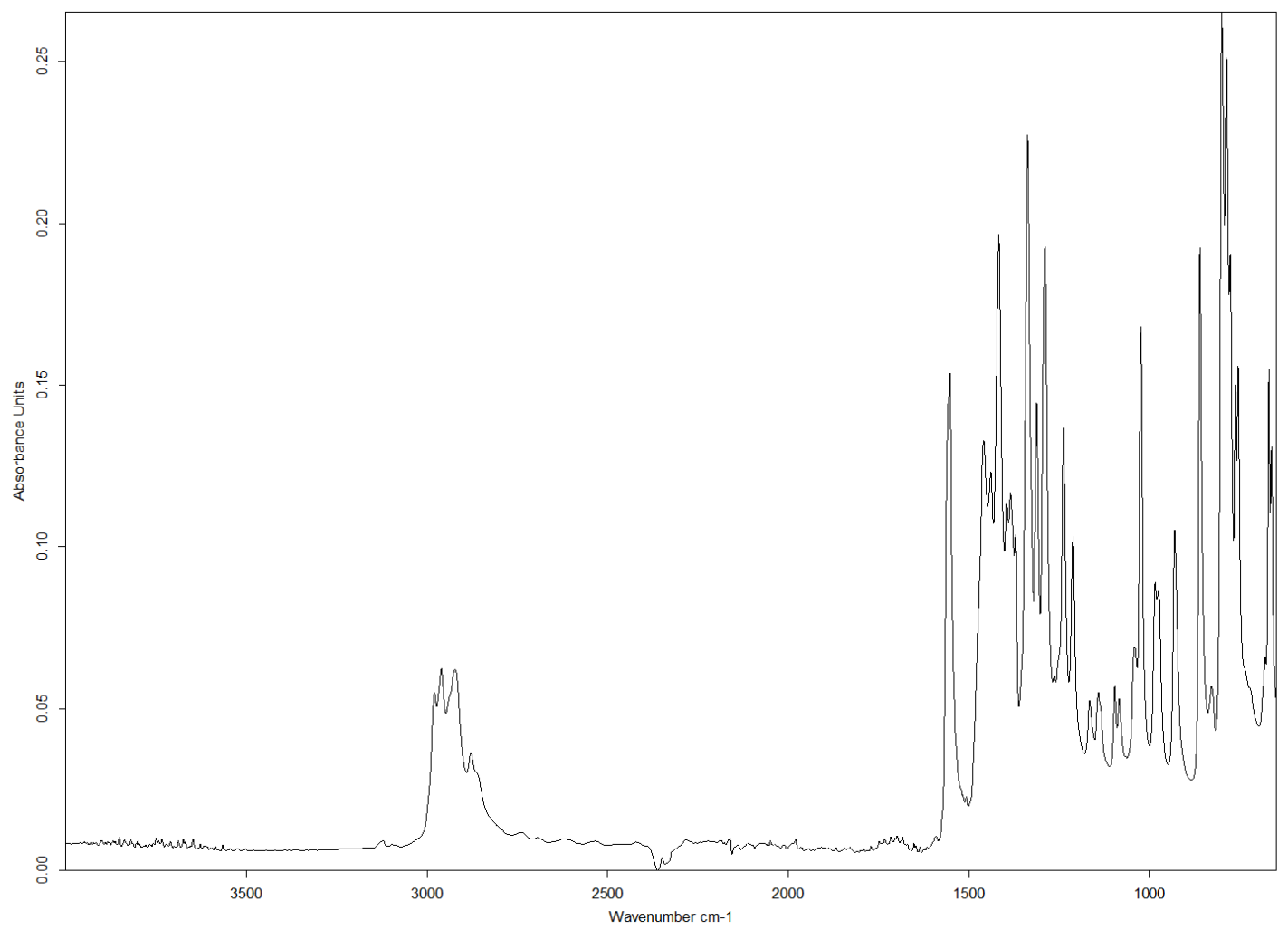

Figure S3.64 IR (Diamond ATR) spectrum of 1,1-bis(3,5-dimethylpyrazol-1-yl)propane<smiles>Cc1cc(C)n(C(=O)n2nc([N+](=O)[O-])cc2[N+](=O)[O-])n1</smiles><smiles>O=CC1CCCCC1</smiles>
$60^{\circ} \mathrm{C}, 10 \%$ DMAP THF, $24 \mathrm{~h}$<smiles>Cc1cc(C)n(C(C2CCCCC2)n2nc([N+](=O)[O-])cc2C)n1</smiles>

bis(3-phenylpyrazol-1-yl)cyclohexylmethane. 


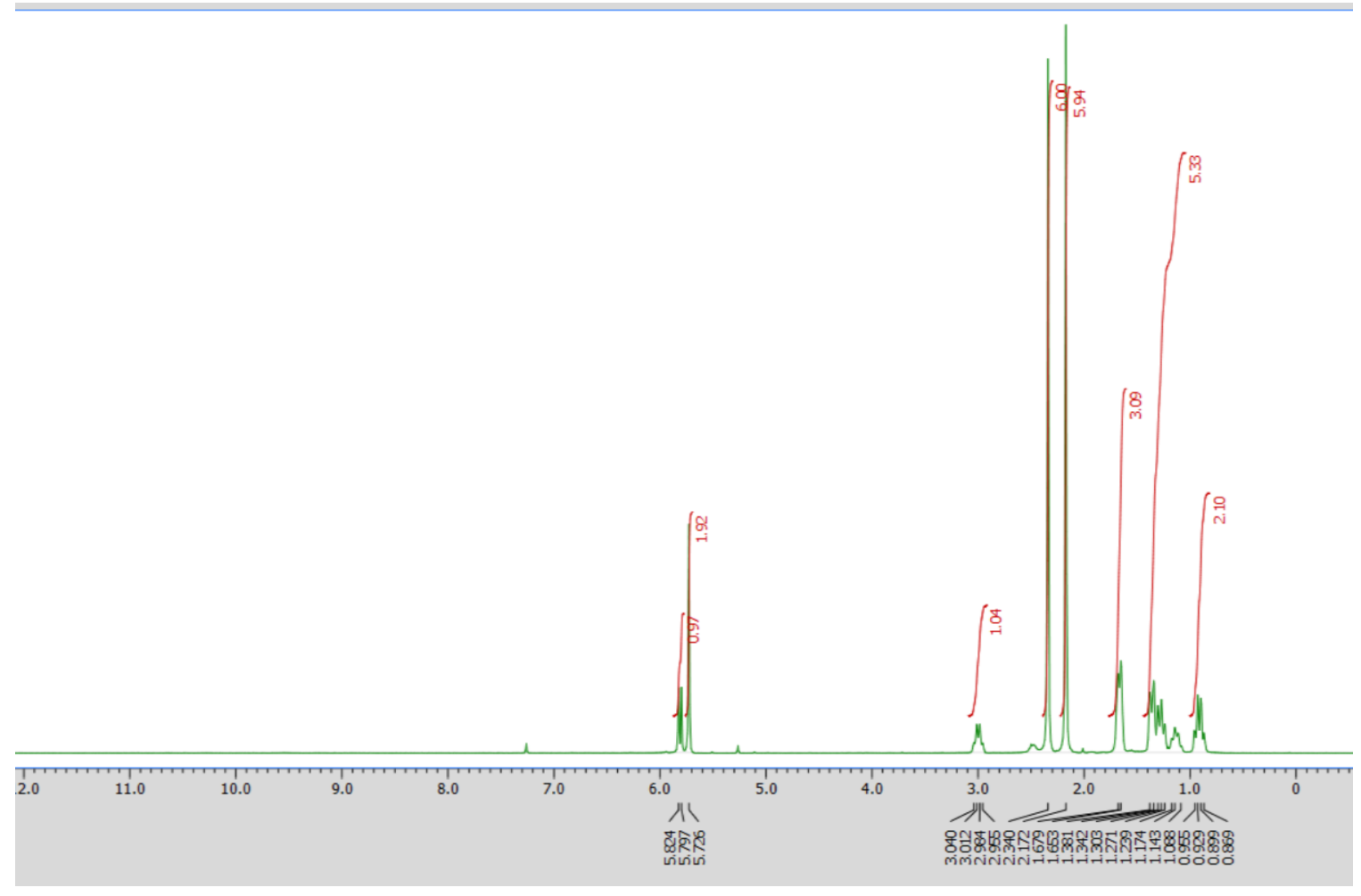

Figure $\mathrm{S} 3.65{ }^{1} \mathrm{H}$ NMR $\left(400 \mathrm{MHz}, \mathrm{CDCl}_{3}\right)$ spectrum of bis(3,5-dimethylpyrazol-1yl)cyclohexylmethane.

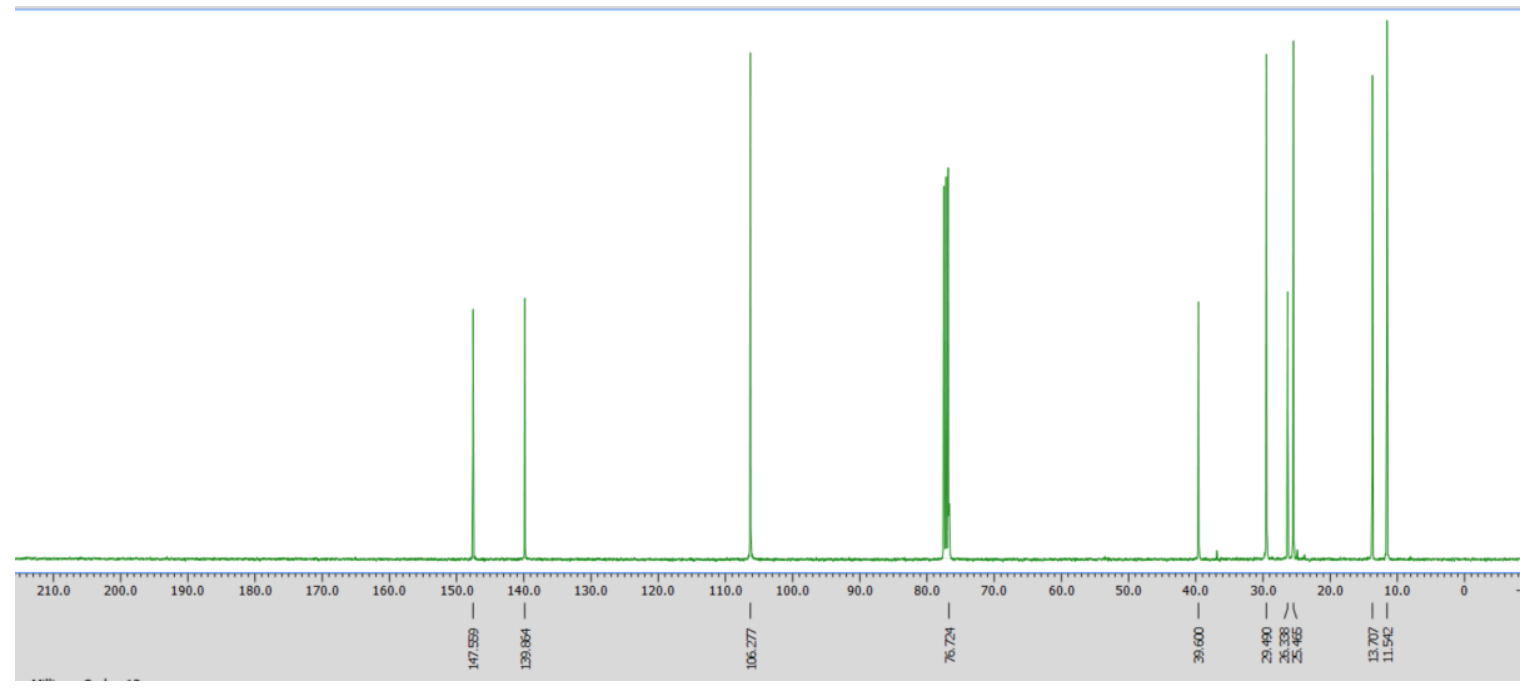

Figure $\mathrm{S} 3.66{ }^{13} \mathrm{C}$ NMR $\left(4101 \mathrm{MHz}, \mathrm{CDCl}_{3}\right)$ spectrum of bis(3,5-dimethylpyrazol-1yl)cyclohexylmethane. 


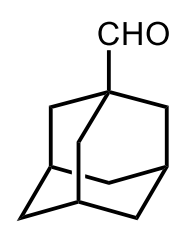<smiles>Cc1cc(C)n(C(=O)n2nc(C)cc2C)n1</smiles>

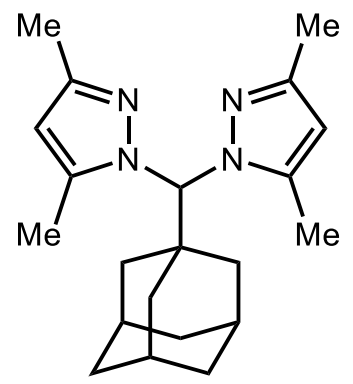

1-bis(3,5-dimethylpyrazol-1-yl)methyladamantane.

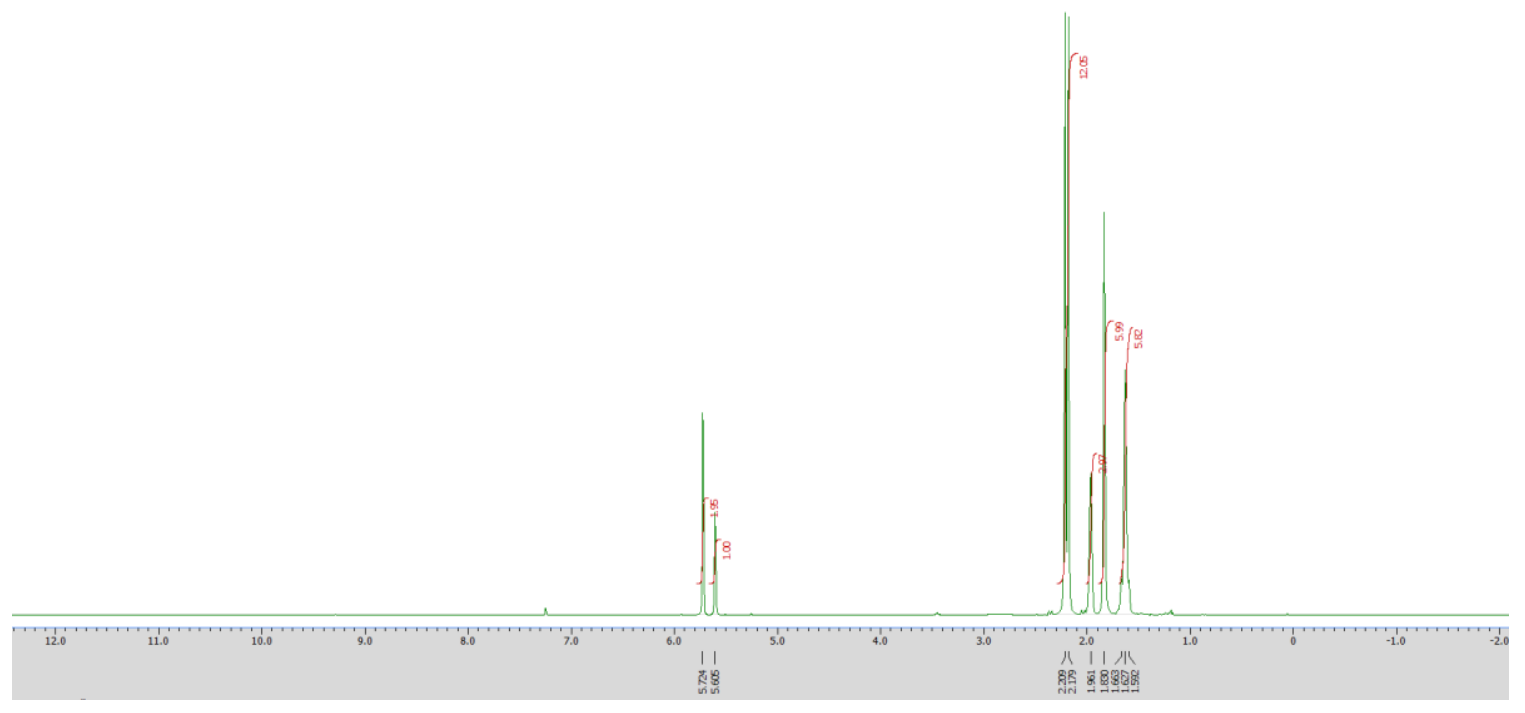

Figure $\mathrm{S} 3.67{ }^{1} \mathrm{H}$ NMR $\left(400 \mathrm{MHz}, \mathrm{CDCl}_{3}\right)$ spectrum of 1-bis(3,5-dimethylpyrazol-1yl)methyladamantane

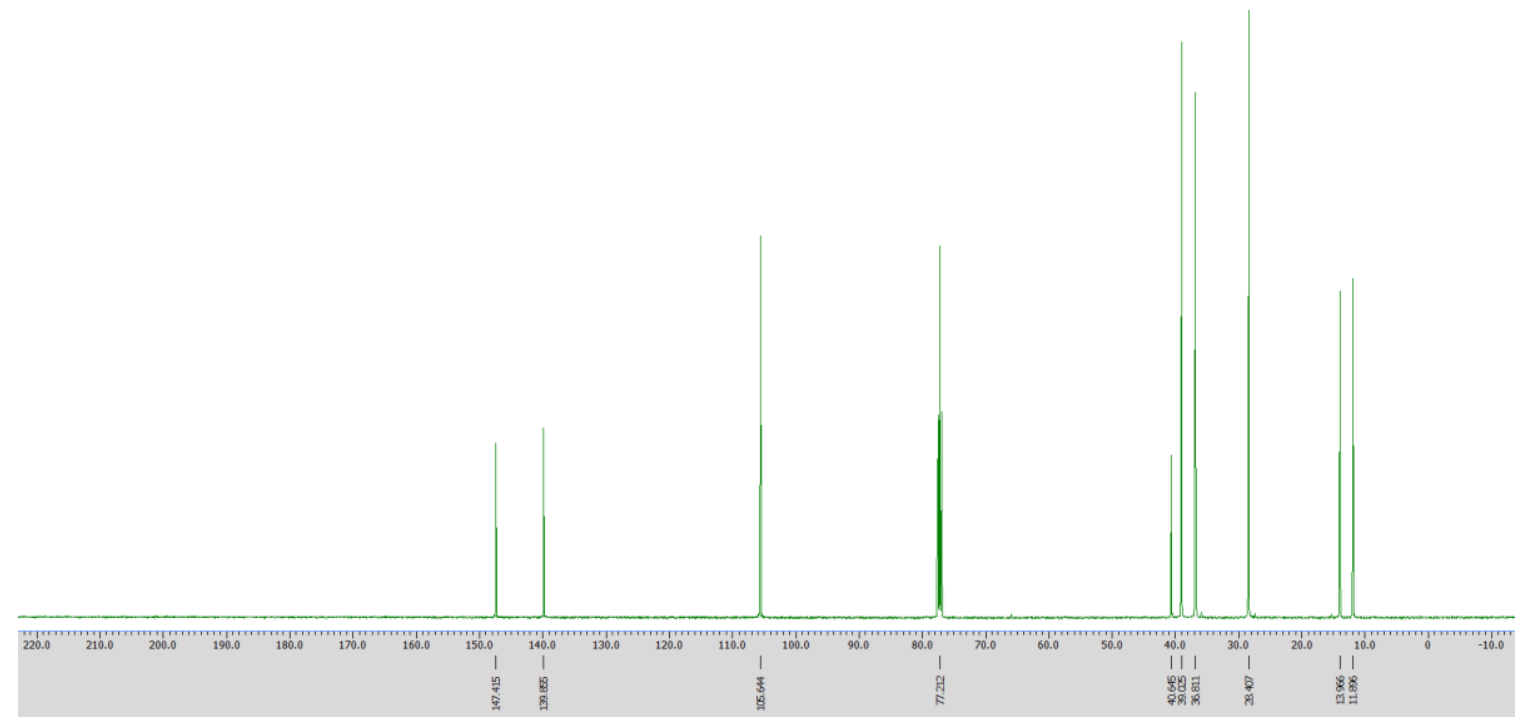


Figure $\mathrm{S} 3.68{ }^{13} \mathrm{C}$ NMR (101 MHz, $\mathrm{CDCl}_{3}$ ) spectrum of 1-bis(3,5-dimethylpyrazol-1yl)methyladamantane

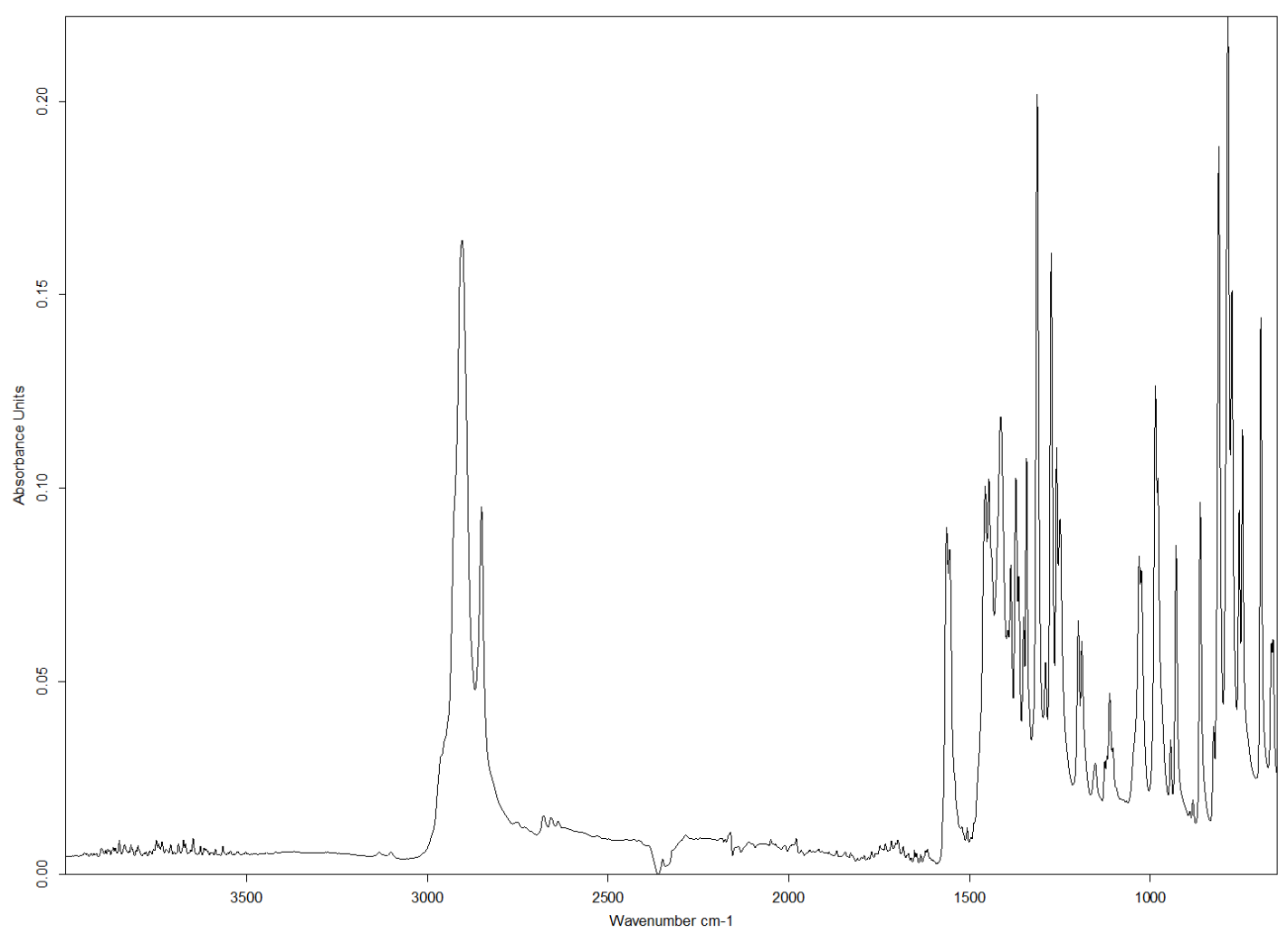

Figure S3.69 IR (Diamond ATR) spectrum of 1-bis(3,5-dimethylpyrazol-1yl)methyladamantane<smiles>O=CCc1ccccc1</smiles><smiles>Cc1cc(C)n(C(=O)n2nc([N+](=O)[O-])cc2C)n1</smiles><smiles>Cc1cc(C)n(C(Cc2ccccc2)n2nc(C)cc2C)n1</smiles>

1-phenyl-2,2-bis(3,5-dimethylpyrazol-1-yl)ethane. 


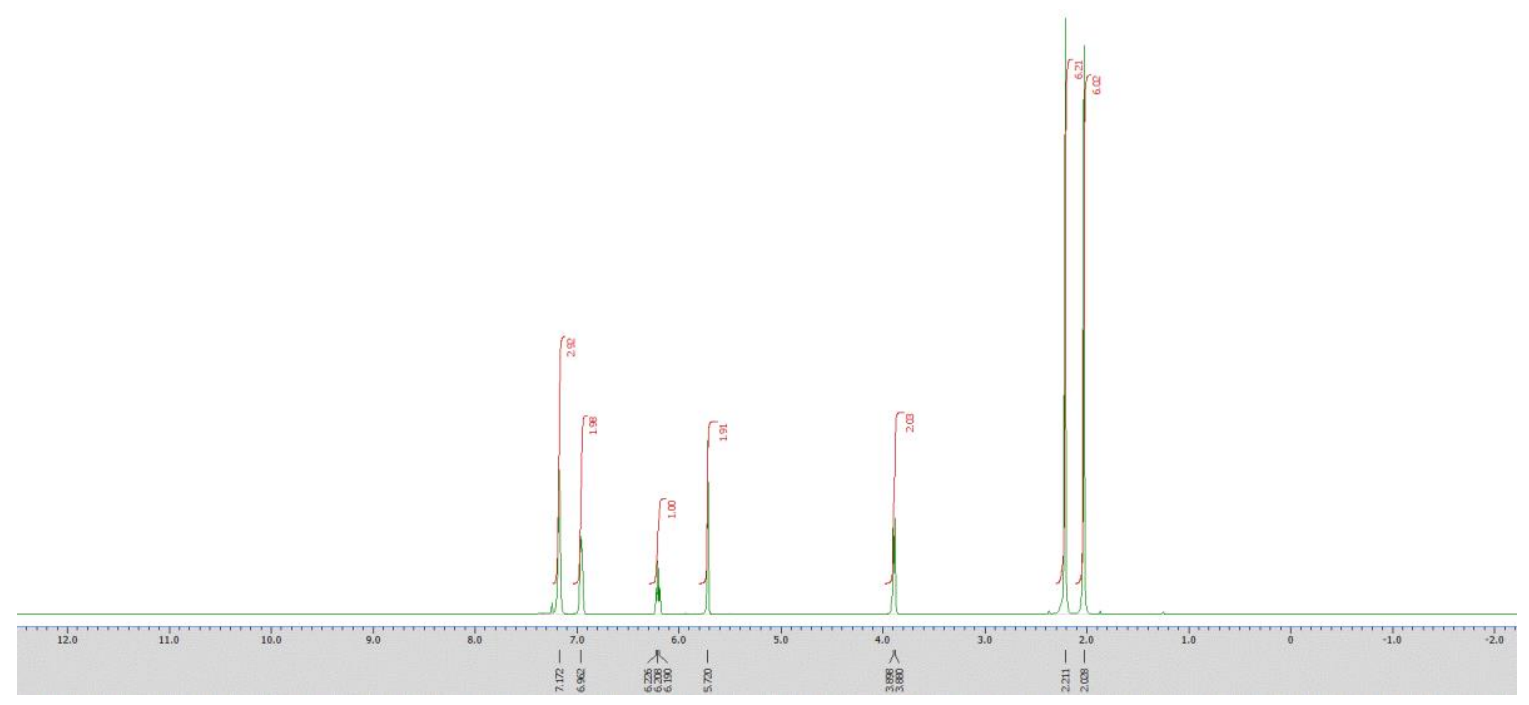

Figure $\mathrm{S} 3.70 \quad{ }^{1} \mathrm{H} \quad \mathrm{NMR} \quad\left(400 \mathrm{MHz}, \mathrm{CDCl}_{3}\right)$ spectrum of 1-phenyl-2,2-bis(3,5dimethylpyrazol-1-yl)ethane

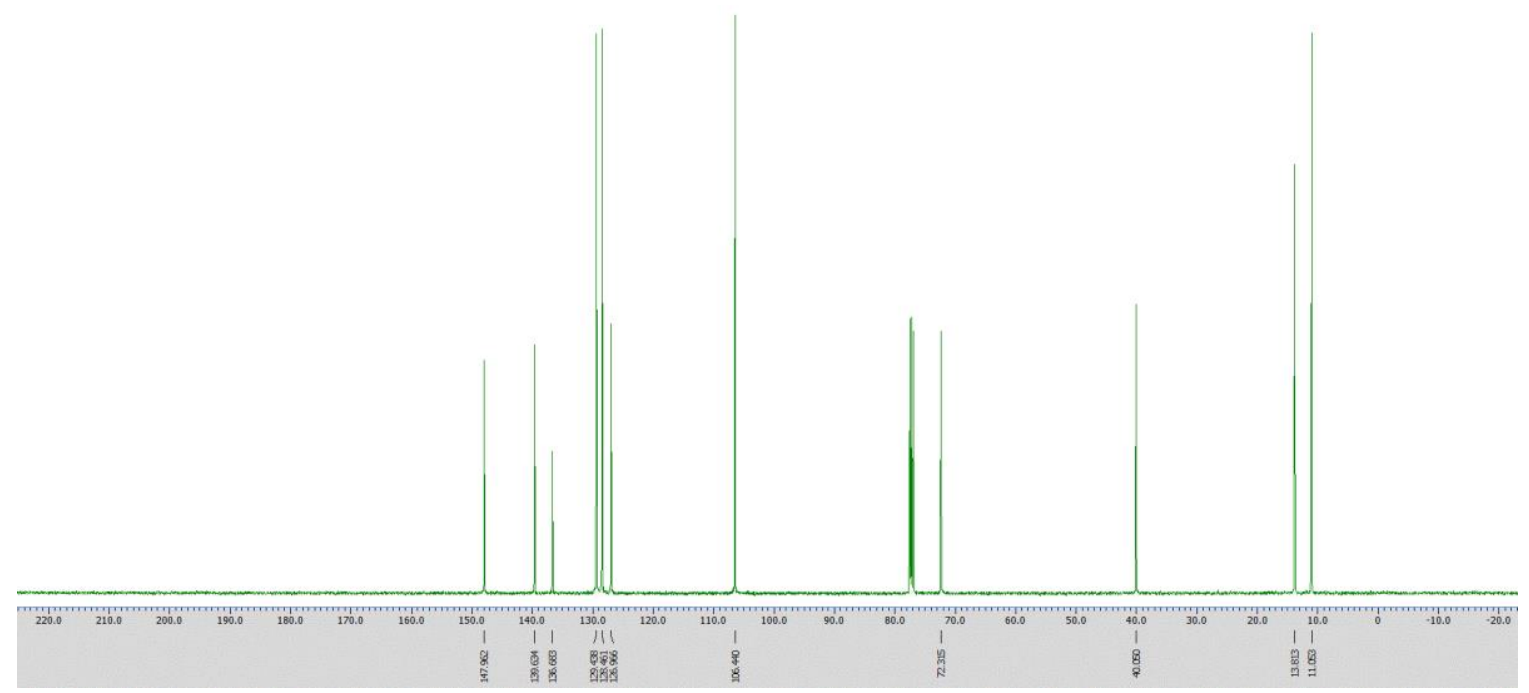

Figure $\mathrm{S} 3.71{ }^{13} \mathrm{C}$ NMR $\left(101 \mathrm{MHz}, \mathrm{CDCl}_{3}\right)$ spectrum of 1-phenyl-2,2-bis(3,5dimethylpyrazol-1-yl)ethane 
<smiles>O=Cc1ccccn1</smiles><smiles>Cc1cc(C)n(C(=O)n2nc(C)cc2C)n1</smiles>

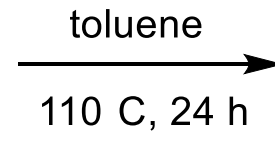<smiles>Cc1cc(C)n(C(c2ccccn2)n2nc(C)cc2C)n1</smiles>

2-bis(3,5-dimethylpyrazo1-1-yl)methylpyridine.

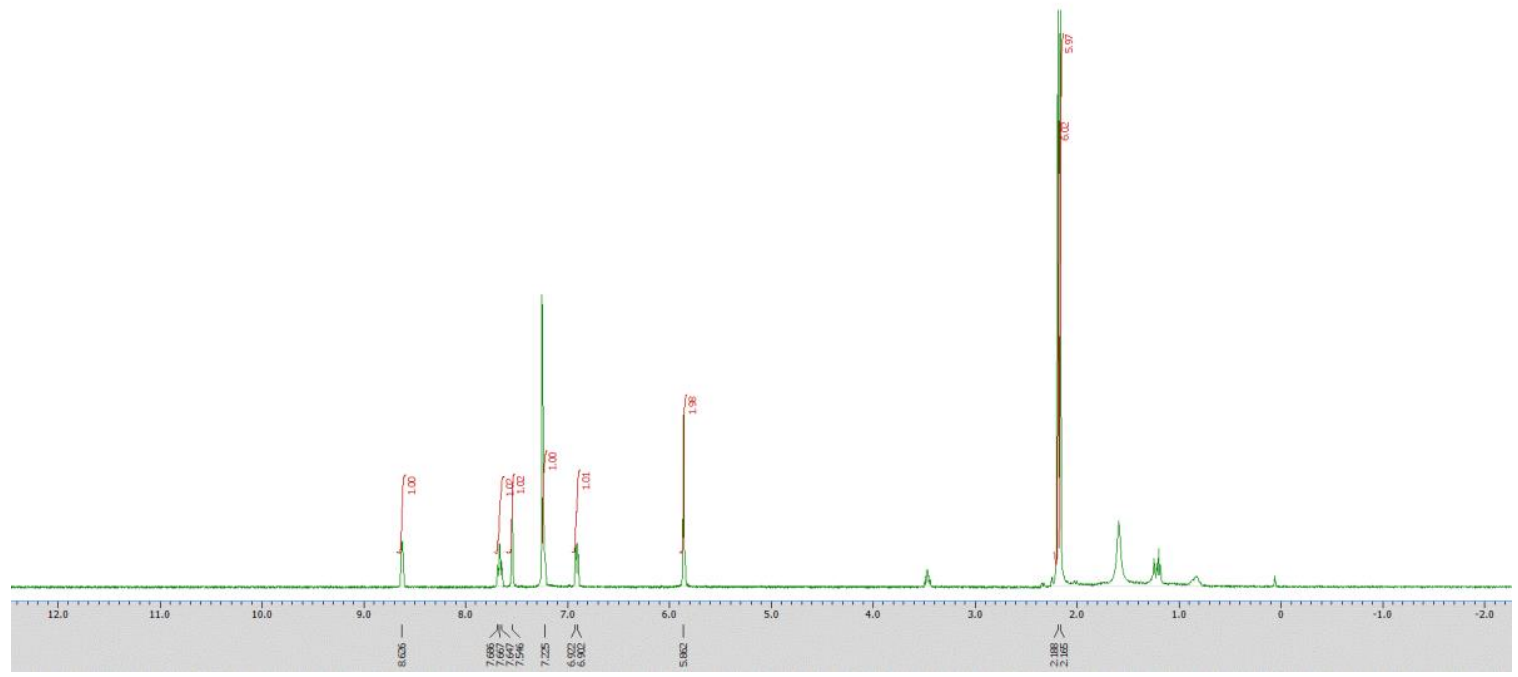

Figure $\mathrm{S} 3.72{ }^{1} \mathrm{H}$ NMR (400 $\left.\mathrm{MHz}, \mathrm{CDCl}_{3}\right)$ spectrum of 2-bis(3,5-dimethylpyrazol-1yl)methylpyridine.

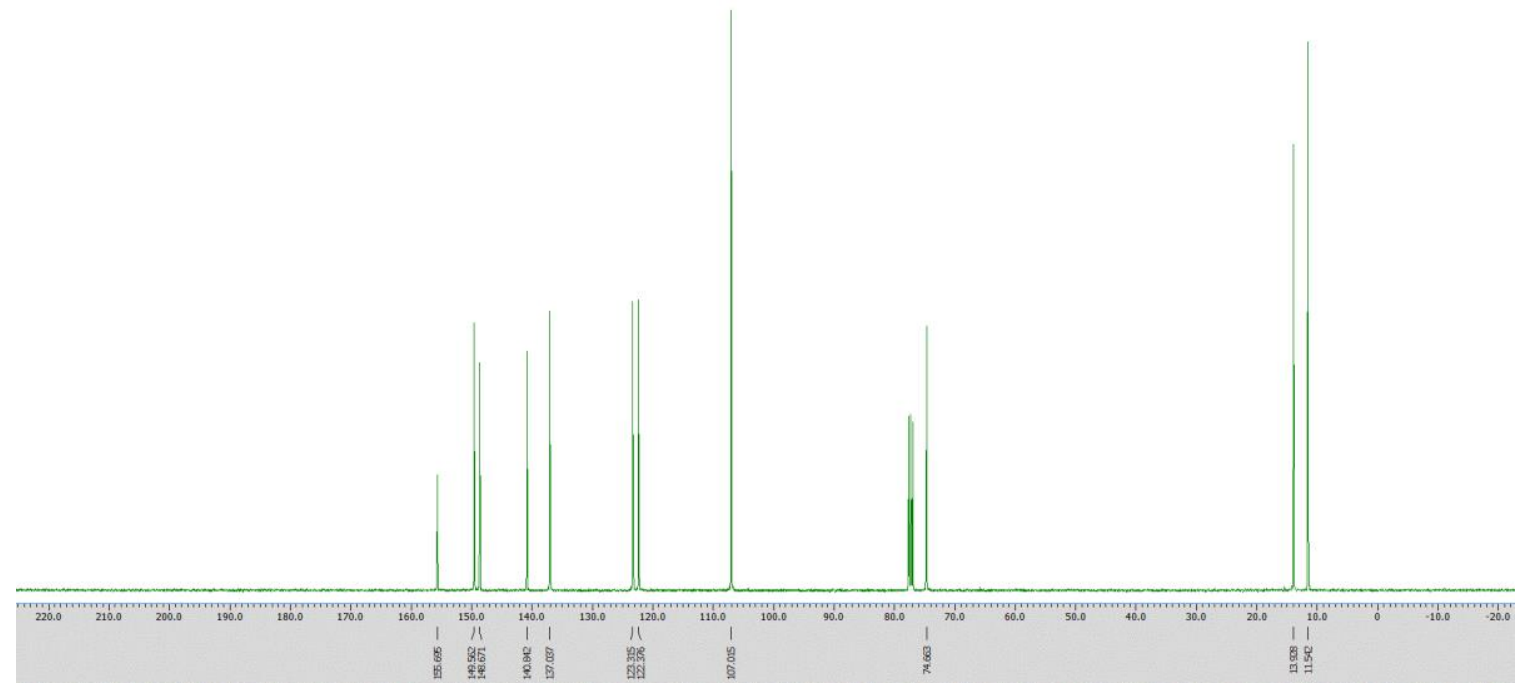


Figure $\mathrm{S} 3.73{ }^{13} \mathrm{C}$ NMR (101 MHz, $\mathrm{CDCl}_{3}$ ) spectrum of 2-bis(3,5-dimethylpyrazol-1yl)methylpyridine<smiles>O=Cc1ccccn1</smiles><smiles>Cc1cc(C)n(C(=O)n2nc(C)cc2C)n1</smiles>

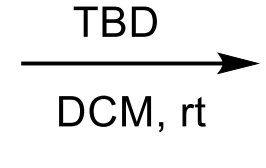<smiles>Cc1cc(C)n(C(=O)OC(c2ccccn2)n2nc(C)cc2C)n1</smiles>

Isolation of 1-(3,5-dimethylpyrazol-1-yl)-(2-pyridinyl)methyl 3,5-dimethylpyrazolecarboxylate (9).

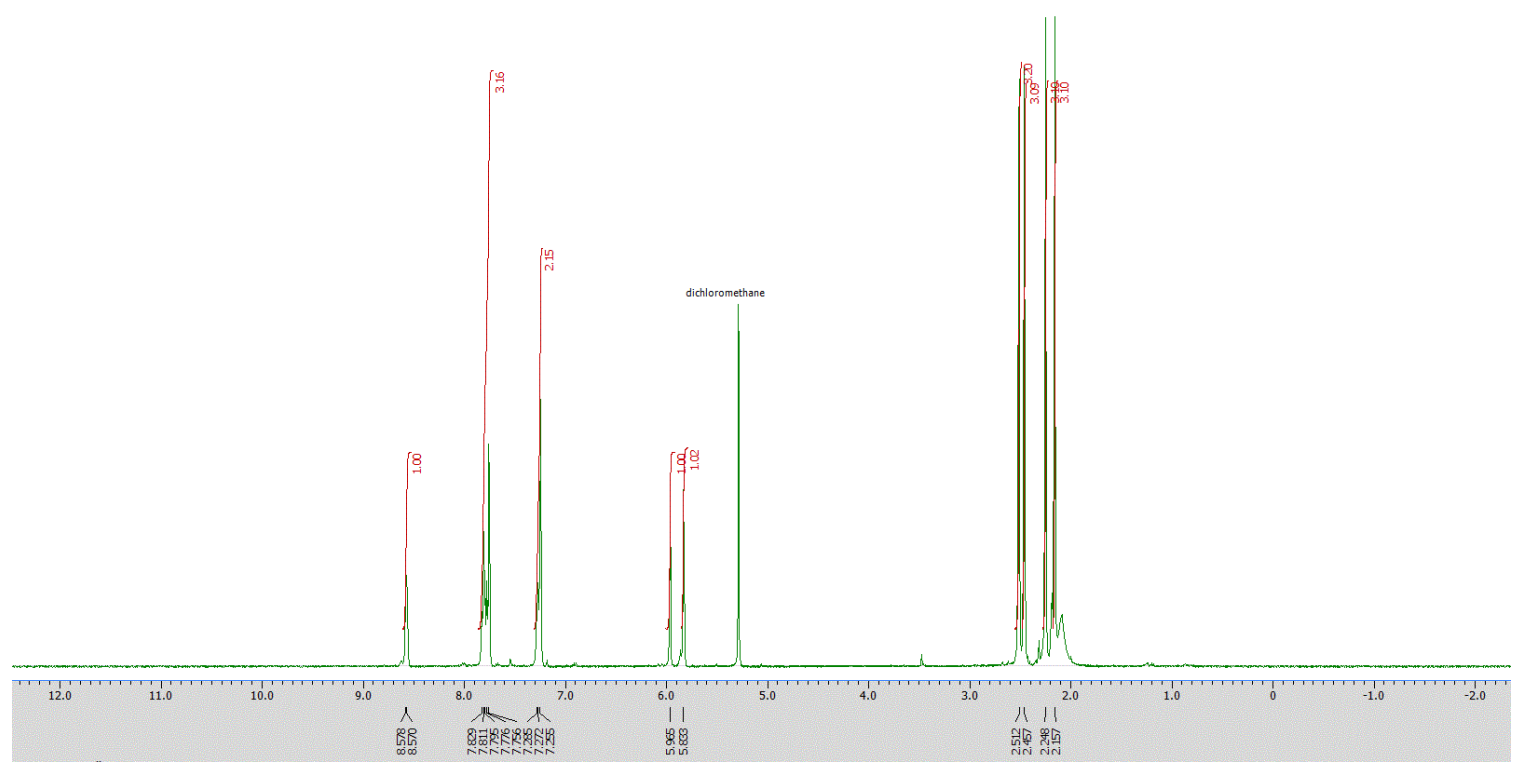

Figure $\mathrm{S} 3.74{ }^{1} \mathrm{H}$ NMR $\left(400 \mathrm{MHz}, \mathrm{CDCl}_{3}\right)$ spectrum of 1-(3,5-dimethylpyrazol-1-yl)-(2pyridinyl)methyl 3,5-dimethylpyrazole-carboxylate (9) 


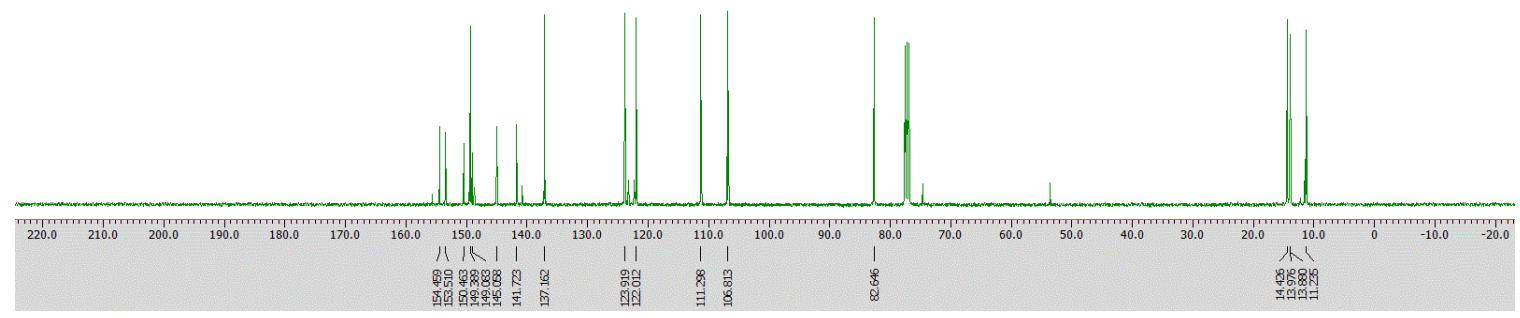

Figure $\mathrm{S} 3.75{ }^{13} \mathrm{C}$ NMR (101 MHz, $\left.\mathrm{CDCl}_{3}\right)$ spectrum of 1-(3,5-dimethylpyrazol-1-yl)-(2pyridinyl)methyl 3,5-dimethylpyrazole-carboxylate (4)

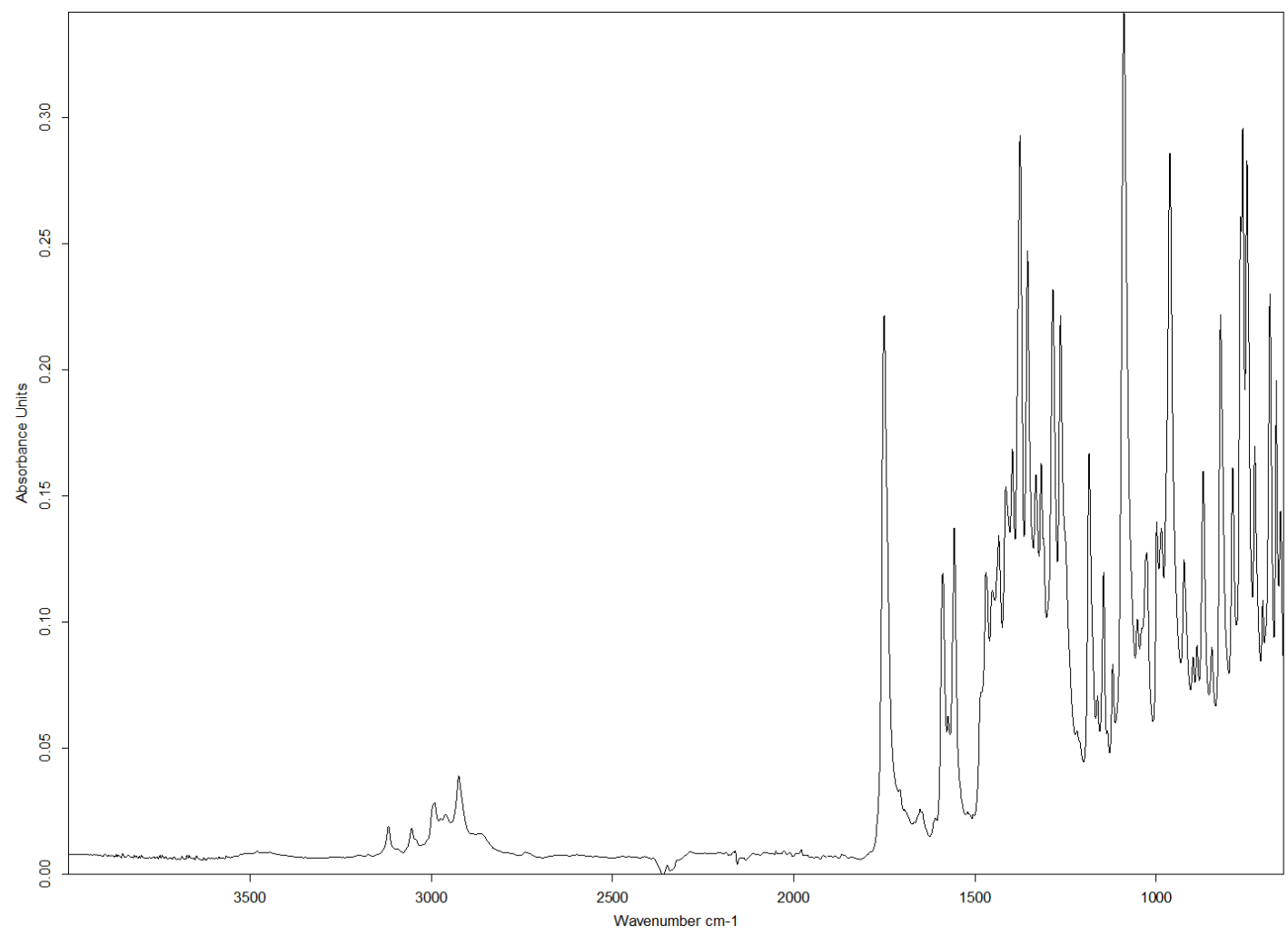

Figure S3.76 IR (Diamond ATR) spectrum of 1-(3,5-dimethylpyrazol-1-yl)-(2pyridinyl)methyl 3,5-dimethylpyrazole-carboxylate (9) 


\section{Initial Optimization Studies in Toluene}

Table S4.1. Initial results for catalyst screen in toluene for the synthesis of bis(pyrazolyl)alkane 3.<smiles>Cc1cc(C)n(C(=O)n2nc(C)cc2C)n1</smiles>
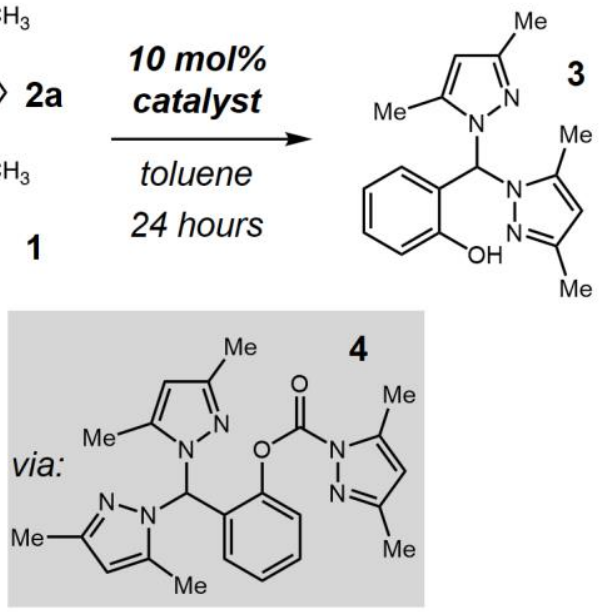

\begin{tabular}{|c|c|c|c|}
\hline Entry & Catalyst & Yield $3^{\mathrm{a}}$ & Yield $4^{\mathrm{a}}$ \\
\hline 1 & - & $27 \%$ & $33 \%$ \\
\hline 2 & DMAP & $48 \%$ & $14 \%$ \\
\hline 3 & triphenylphosphine & $26 \%$ & $30 \%$ \\
\hline 4 & 1-methylimidazole & $37 \%$ & $40 \%$ \\
\hline 5 & $\mathrm{DBU}$ & $68 \%$ & $<1 \%$ \\
\hline 6 & TBD & $62 \%$ & $2 \%$ \\
\hline 7 & quinuclidine & $81 \%$ & $7 \%$ \\
\hline 8 & potassium 2-pyridone & $29 \%$ & $40 \%$ \\
\hline 9 & $\mathrm{BF}_{3} \cdot \mathrm{OEt}_{2}$ & not observed & not observed \\
\hline 10 & $\mathrm{ZnCl}_{2}$ & $13 \%$ & $20 \%$ \\
\hline 11 & $\mathrm{TiCl}_{4}$ & not observed & not observed \\
\hline 12 & $p$-TsOH & $15 \%$ & $18 \%$ \\
\hline
\end{tabular}

Reactions were run using 1:1 ratio of 1:2a at $0.5 \mathrm{mmol}$ of $\mathbf{1}$ in $3 \mathrm{~mL}$ anhydrous toluene at $110{ }^{\circ} \mathrm{C}$ unless noted otherwise. ${ }^{\text {a }}$ Yields by ${ }^{1} \mathrm{H}-$ NMR. 
5. Hammett Analysis and Mechanistic Data-Hammett Analysis was performed as competition experiments between a set of five 4-substituted benzaldehydes (4methoxybenzaldehyde, 4-methylbenzaldehyde, 4-chlorobenzaldehyde, 4fluorobenzaldehyde, and 4-nitrobenzaldehyde.) and benzaldehyde. The reaction procedure is a modification to the general procedure: DMAP $(3.0 \mathrm{mg}, 0.025 \mathrm{mmol})$ was added to a solution of $\operatorname{bis}(3,5$-dimethylpyrazol-1-yl)methanone (55 mg, $0.25 \mathrm{mmol}$ ), benzaldehyde $(0.128 \mathrm{~mL}, 1.25 \mathrm{mmol})$, and 4-substituted benzaldehyde (1.25 mmol) under nitrogen. The solution was stirred at $60{ }^{\circ} \mathrm{C}$ for $24 \mathrm{~h}$, and then all volatiles were distilled off in vacuo at $60^{\circ} \mathrm{C}$. After cooling to room temperature, 1,4-dioxane $(10 \mu \mathrm{L})$ was added as internal standard and an aliquot was dissolved in DMSO-d6 for assay.<smiles>[X]c1ccc(C=O)cc1</smiles><smiles>[X]c1ccc(C(n2nc(C)cc2C)n2nc(C)cc2C)cc1</smiles>

Table S5.1. Hammett Analysis parameters for DMAP-catalyzed condition

\begin{tabular}{cccc}
\hline & $\mathbf{p}$ & $\mathbf{k x} / \mathbf{k}_{\mathbf{H}}$ & $\log \left(\mathbf{k} \mathbf{x} / \mathbf{k}_{\mathbf{H}}\right)$ \\
$-\mathbf{N O 2}$ & 0.75 & 36.4 & 1.56 \\
$-\mathbf{C l}$ & 0.21 & 2.61 & 0.417 \\
$-\mathbf{F}$ & 0.04 & 1.52 & 0.181 \\
$-\mathbf{M e}$ & -0.19 & 0.384 & -0.416 \\
- OMe & -0.4 & 0.179 & -0.747 \\
\hline
\end{tabular}

$\sigma=+2.04, \mathrm{R}^{2}=0.995$ 


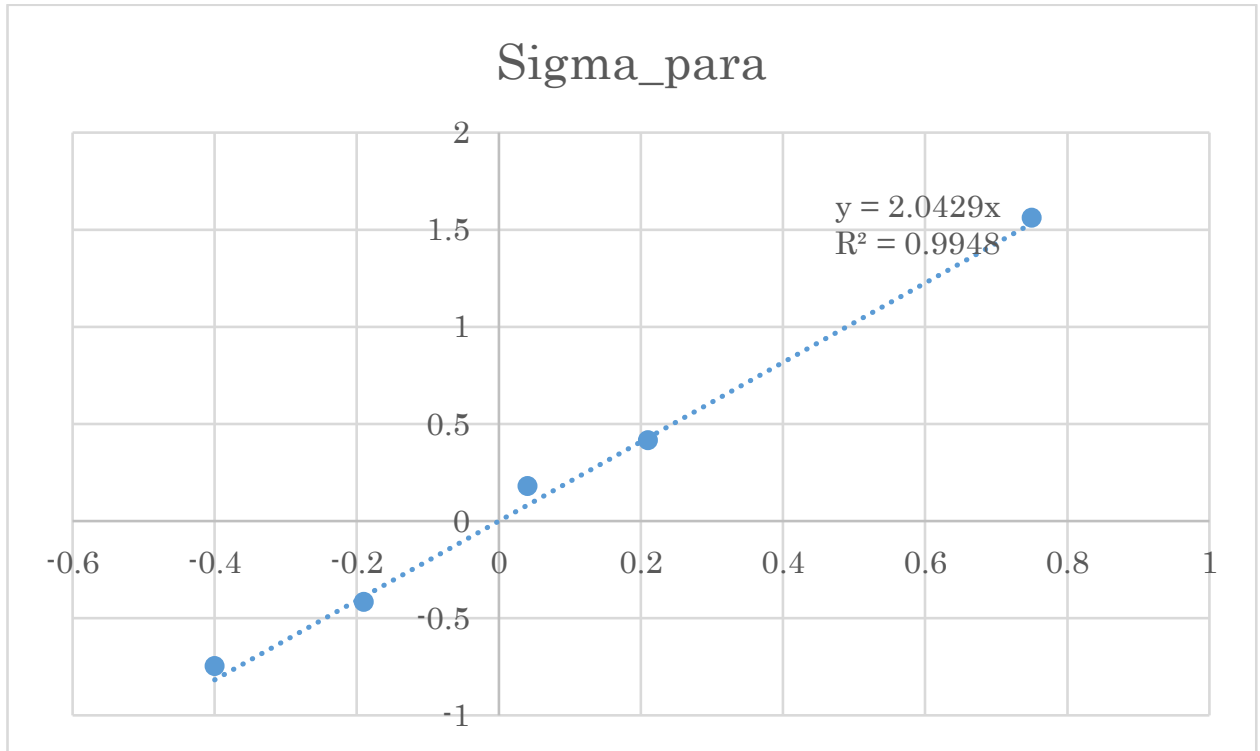

Figure S5.1. Hammett Analysis plot for DMAP-catalyzed condition<smiles>[X]c1ccc(C=O)cc1</smiles><smiles>[X]c1ccc(C(n2nc(C)cc2C)n2nc(C)cc2C)cc1</smiles>

Table S5.2. Hammett Analysis parameters for catalyst-free condition

\begin{tabular}{cccc}
\hline & $\mathbf{p}$ & $\mathbf{k x} / \mathbf{k} \mathbf{H}$ & $\log (\mathbf{k x} / \mathbf{k H})$ \\
$-\mathbf{N O 2}$ & 0.75 & 15.6 & 1.19 \\
$-\mathbf{C l}$ & 0.21 & 1.81 & 0.259 \\
$-\mathbf{F}$ & 0.04 & 1.15 & 0.061 \\
$-\mathbf{M e}$ & -0.19 & 0.536 & -0.271 \\
$-\mathbf{O M e}$ & -0.4 & 0.346 & -0.460 \\
\hline
\end{tabular}

$\sigma=+1.48, \mathrm{R}^{2}=0.984$ 


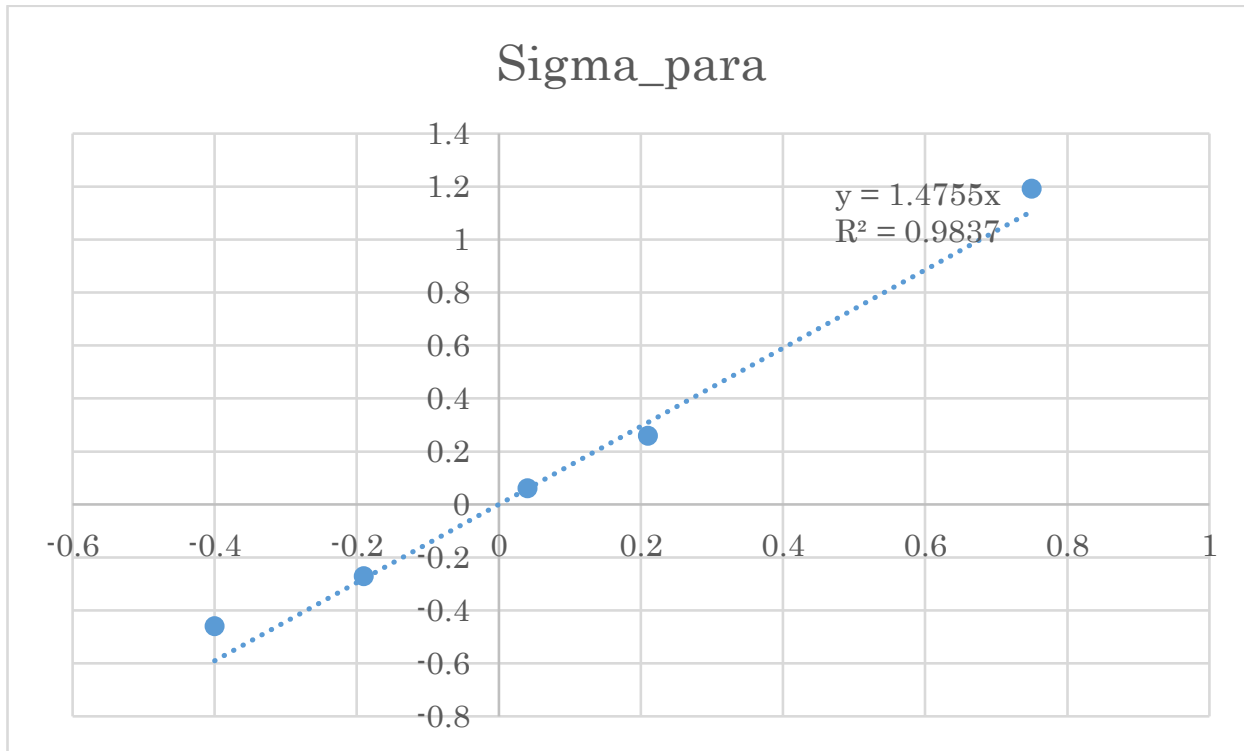

Figure S5.2. Hammett Analysis plot for catalyst-free condition<smiles>O=C(n1cccn1)n1cccn1</smiles><smiles>Cc1cc(C)n(C(=O)n2nc(C)cc2C)n1</smiles>

10 mol\% DMAP

or

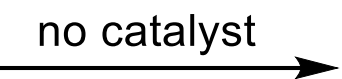

THF

$60{ }^{\circ} \mathrm{C}, 5 \mathrm{~h}$<smiles>Cc1cc(C)n(C(=O)n2cccn2)n1</smiles> 


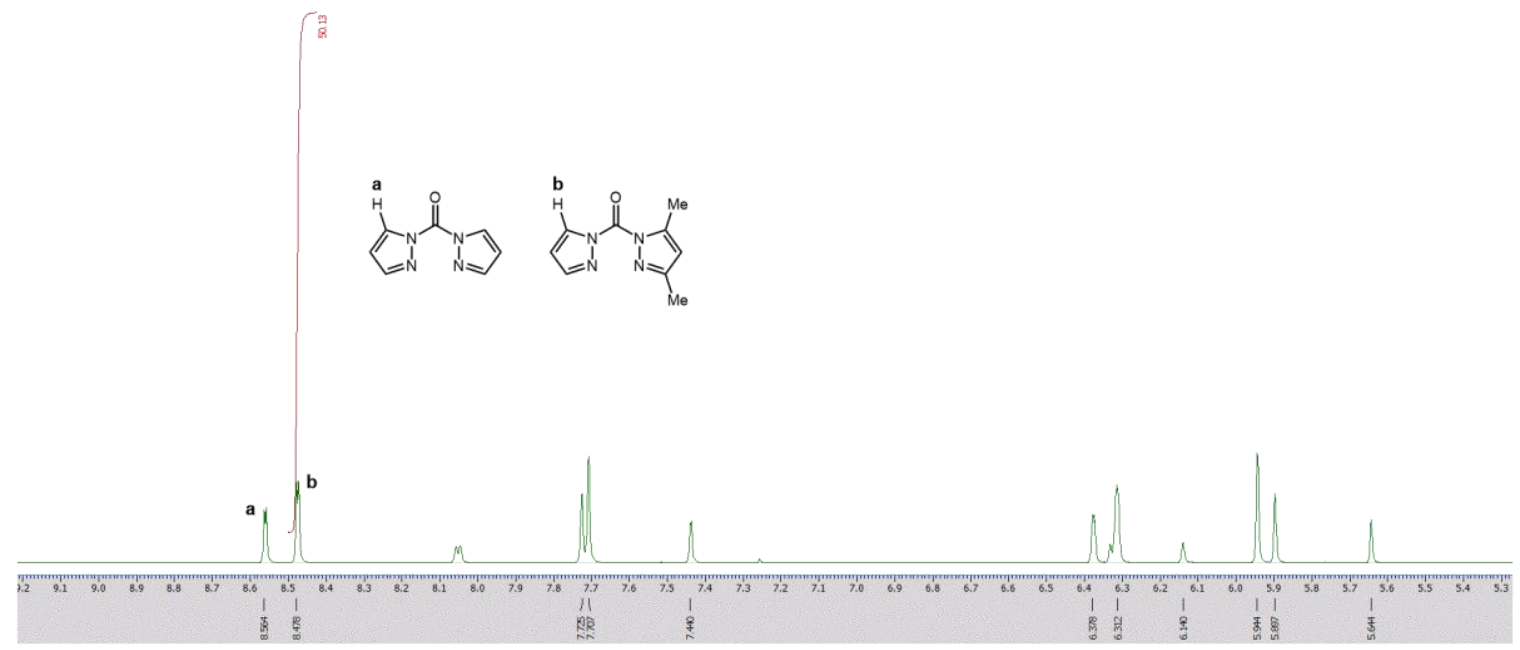

Figure S5. ${ }^{1} \mathrm{H}$ NMR $\left(400 \mathrm{MHz}, \mathrm{CDCl}_{3}\right)$ spectrum of crossover mixture under $10 \mathrm{~mol} \%$ DMAP condition, integrated quantitatively against an internal standard.

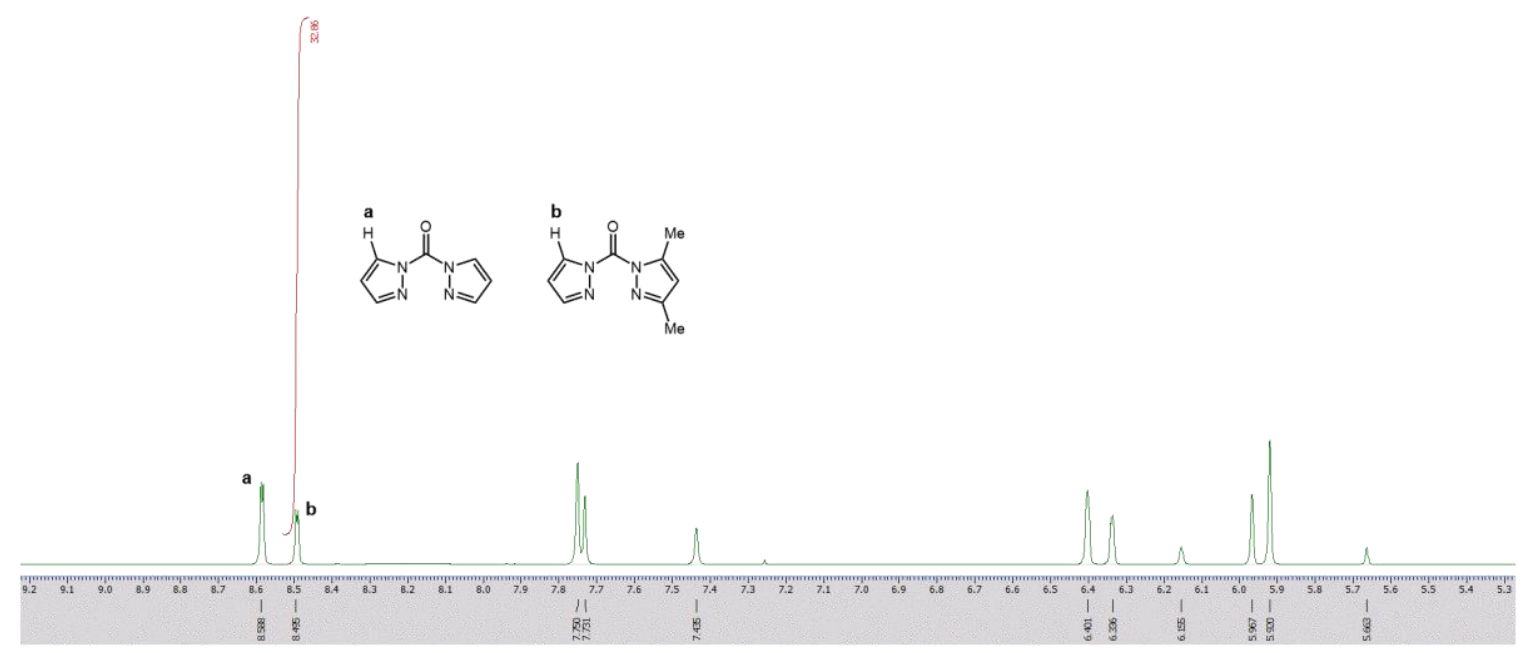

Figure $\mathrm{S} 5.7^{1} \mathrm{H}$ NMR $\left(400 \mathrm{MHz}, \mathrm{CDCl}_{3}\right)$ spectrum of crossover mixture under uncatalyzed condition, integrated quantitatively against an internal standard. 